\title{
COMET:
}

\section{CONSTRAINED OPTIMIZATION OF MULTIPLE-DIMENSIONS FOR EFFICIENT TRAJECTORIES}

\author{
A Thesis \\ presented to \\ the Faculty of California Polytechnic State University, \\ San Luis Obispo
}

\author{
In Partial Fulfillment \\ of the Requirements for the Degree \\ Master of Science in Aerospace Engineering
}

by

Michael Curt Conrad

December 2011 
(C) 2011

Michael Curt Conrad

ALL RIGHTS RESERVED 


\section{COMMITTEE MEMBERSHIP}

TITLE:

AUTHOR:

DATE SUBMITTED:

COMMITTEE CHAIR:

COMMITTEE MEMBER:

COMMITTEE MEMBER:

COMMITTEE MEMBER:
COMET: Constrained Optimization of Multiple-

dimensions for Efficient Trajectories

Michael Curt Conrad

December 2011

Kira Abercromby, $\mathrm{PhD}$.

Eric Mehiel, $\mathrm{PhD}$.

Tina Jameson, $\mathrm{PhD}$.

Erick Sturm, NASA-JPL 


\author{
ABSTRACT \\ COMET: CONSTRAINED OPTIMIZATION OF \\ MULTIPLE DIMENSIONS FOR EFFICIENT \\ TRAJECTORIES \\ Michael Conrad
}

The paper describes the background and concepts behind a master's thesis platform known as COMET (Constrained Optimization of Multiple-dimensions for Efficient Trajectories) created for mission designers to determine and evaluate suitable interplanetary trajectories. This includes an examination of the improvements to the global optimization algorithm, Differential Evolution, through a cascading search space pruning method and decomposition of optimization parameters. Results are compared to those produced by the European Space Agency's Advanced Concept Team's Multiple Gravity Assist Program. It was found that while discrepancies in the calculation of $\Delta \mathrm{V}$ 's for flyby maneuvers exist between the two programs, COMET showed a noticeable improvement in its ability to avoid premature convergence and find highly isolated solutions. 


\section{ACKNOWLEDGMENTS}

I would like to thank Dr. Kira Abercromby of Cal Poly, San Luis Obispo, CA for her guidance and encouragement, not only while mentoring me with my thesis but throughout my academic career. Additionally, I would like to thank Dr. Eric Mehiel, Dr. Tina Jameson, and Dr. Jordi Puig-Suari from the Aerospace Engineering department at Cal Poly, and Erick Sturm from NASA-JPL, for all their advice, suggestions, and critiques. Most importantly, I would like to thank my fiancé, Rachel, for her unconditional love and support throughout this process. 
LIST OF TABLES

Page

LIST OF FIGURES

vii

NOMENCLATURE AND ABBREVIATIONS

viii

INTRODUCTION AND BACKGROUND

$\mathbf{x}$

INTERPLANETARY TRAJECTORY

SEARCH SPACE PRUNING

DIFFERENTIAL EVOLUTION

RESULTS

CASE I: Earth-Jupiter-Saturn(EJS)

CASE II: Earth-Mars-Jupiter-Saturn(EMJS)

CASE III: Earth-Venus-Earth-Jupiter-Saturn(EVEJS)

CASE IV: Earth-Venus-Venus-Earth-Jupiter-Saturn(EVVEJS)

CASE V: Earth-Venus-DSM-Venus-Earth-Jupiter-Saturn(EVDVEJS)

Multiple Linear Regression Analysis 49

COMET'S GRAPHICAL USER INTERFACE 52

CONCLUSION AND FUTURE WORK 55

REFERENCES 61

APPENDIX A: INITIAL PROBLEM STRUCTURES

64

APPENDIX B: COMET'S LAMBERT'S PROBLEM FUNCTION 65

APPENDIX C: MULTIPLE LINEAR REGRESSION ANALYSIS 70

74 


\section{LIST OF TABLES}

Page

Table 1: Date spacing and index skipping values to unequal grid spacing ratios

Table 2: Bounds and best known solutions for EJS test case

Table 3: Bounds and best known solutions for EMJS test case

Table 4: Bounds and best known solutions for EVEJS test case

Table 5: Bounds and best known solutions for EVVEJS test case

Table 6: Bounds and best known solutions for EVDVEJS test case

Table 7: Multiple Regression Designs and Simplified Regression Equations

Table 8: Test case results in $\mathrm{km} / \mathrm{s}$ 


\section{LIST OF FIGURES}

Figure 1: Earth to Venus porkchop plot

Figure 2: Illustration of flyby values $\mathbf{r}_{p}$ and $\delta$ in B-plane

Figure 3: Random generation of points in a search space before pruning

Figure 4: Random generation of points in a search space after pruning

Figure 5: Illustration of unequal grid spacing on date indexing

Figure 6: Visualization of valid search space regions for a flyby

Figure 7: Illustration of pruning invalid trajectory legs and shifting of backtracking indexes

Figure 8: Differential evolution process

Figure 9: Initial population members for Differential Evolution

Figure 10: Selection of base member and difference vector

Figure 11: Generation of mutation member

Figure 12: Selection process when mutation replaces original population member

Figure 13: Selection process when original population member does not get replaced by mutation

Figure 14: Examples of (a) binomial and (b) exponential crossover

Figure 15: Valid Earth launch search space regions for EJS

Figure 16: Valid Jupiter flyby search space regions for EJS

Figure 17: Residual plot of best known solutions for EJS

Figure 18: Valid Earth launch search space regions for EMJS

Figure 19: Valid Mars flyby search space regions for EMJS 
Figure 21: Residual plot of best known solutions for EMJS

Figure 22: Valid Earth launch search space regions for EVEJS

Figure 23: Valid Venus flyby search space regions for EVEJS

Figure 24: Valid Earth flyby search space regions for EVEJS

Figure 25: Valid Jupiter flyby search space regions for EVEJS

Figure 26: Residual plot of best known solutions for EVEJS

Figure 27: Valid Earth launch search space regions for EVVEJS

Figure 28: Valid Venus flyby search space regions for EVVEJS

Figure 29: Valid Venus flyby search space regions for EVVEJS

Figure 30: Valid Earth flyby search space regions for EVVEJS

Figure 31: Valid Jupiter flyby search space regions for EVVEJS

Figure 32: Residual plot of best known solutions for EVVEJS

Figure 33: Residual plot of best known solutions for EVDVEJS without decomposing

Figure 34: Residual plot of best known solutions for EVDVEJS with decomposing

Figure 35: Multiple regression data set and regression slopes

Figure 36: COMET during Set-Up for EVVEJS problem set

Figure 37: COMET with Prune tab selected for EVVEJS problem set

Figure 38: COMET with Optimization tab selected for EVVEJS problem set

Figure 39: COMET with Visualize tab selected for EVVEJS problem set 


\section{Nomenclature and Abbreviations}

\begin{tabular}{|c|c|}
\hline B-plane & $=$ Plane of hyperbolic trajectory during a flyby \\
\hline COMET & $=$ Constrained Optimization of Multiple-dimension for Efficient \\
\hline & Trajectories \\
\hline $\mathrm{Cr}$ & $=$ Mutation cross over ratio \\
\hline$D E$ & $=$ Differential evolution global optimization \\
\hline$D S M$ & $=$ Deep space maneuver \\
\hline EJS & $=$ Earth-Jupiter-Saturn trajectory problem \\
\hline EMJS & $=$ Earth-Mars-Jupiter-Saturn trajectory problem \\
\hline EVEJS & $=$ Earth-Venus-Earth-Jupiter-Saturn trajectory problem \\
\hline EVVEJS & $=$ Earth-Venus-Venus-Earth-Jupiter-Saturn trajectory problem \\
\hline$E S A$ & $=$ European Space Agency \\
\hline$F$ & $=$ Mutation scaling factor \\
\hline$G U I$ & $=$ Graphical user interface \\
\hline$J$ & $=$ Cost \\
\hline$J 2000$ & $=$ Julian date compared to Jan 1, 2000 at 12:00:00 \\
\hline$M G A$ & $=$ Multiple Gravity Assist trajectory design optimizer \\
\hline$N p$ & $=$ Number of members in optimization population \\
\hline$T$ & $=$ Time since epoch \\
\hline$T O F$ & $=$ Time-of-flight \\
\hline$V$ & $=$ Velocity \\
\hline$W$ & $=$ Total weight comparing mission time to total change in velocity \\
\hline$a$ & $=$ Semi-major axis \\
\hline$d 0$ & $=$ Epoch or launch date \\
\hline$e$ & $=$ Eccentricity \\
\hline$\hat{n}$ & $=$ Vector normal to plane \\
\hline$r_{p}$ & $=$ Periapse radius \\
\hline
\end{tabular}




$\begin{array}{ll}v & =\text { Speed } \\ w & =\text { Weighting on importance of individual maneuver } \\ \Delta V & =\text { Change in velocity } \\ \alpha & =\text { Percentage of time-of-flight where a deep space maneuver occurs } \\ \beta & =\text { Rotation angle of B-plane } \\ \delta & =\text { Turn angle of hyperbolic flyby } \\ \mu & =\text { Gravitational parameter of central body }\end{array}$

\section{SUBSCRIPTS:}

$\begin{array}{ll}\text { hyp } & =\text { Hyperbolic } \\ p & =\text { Pertaining to periapse } \\ \text { planet } & =\text { Value associated with flyby planet } \\ \infty & =\text { Value at sphere of influence }\end{array}$

\section{SUPERSCRIPTS:}

$\begin{array}{ll}- & =\text { Approach } \\ + & =\text { Departure }\end{array}$




\section{INTRODUCTION AND BACKGROUND}

Space exploration started simply as a race to demonstrate power and superiority, and has now shifted its focus on pushing the bounds of scientific discovery. Unfortunately, as public interest waned over the past few decades, the funding for these missions drastically reduced. Consequently, space agencies around the world now look for new ways to improve the overall quality and quantity of returned scientific data from each mission, while cutting costs as much as possible. Achieving these goals often results from reducing a spacecraft's propellant mass, allowing mission designers to dedicate more mass to scientific payloads or potentially to use a smaller class of launch vehicles. However, propellant plays a critical role in missions and reducing it has serious implications. The easy-to-use program COMET (Constrained Optimization of Multipledimensions for Efficient Trajectories), enables mission designers to continually improve and reduce the estimations and margins placed on propellant through trajectory optimization.

In the early 1960's, it was proposed that using the gravitational influence of one planet to "sling-shot" the spacecraft towards another could significantly reduce the total change in velocity, $\Delta \mathrm{V}$, provided by the spacecraft propulsion system, equating to a lower overall propellant requirement. ${ }^{[4]}$ Verified by multiple missions since then, these maneuvers-more accurately referred to as gravity assisted flybys-result from a momentum exchange between the planet and the spacecraft. Due to the significantly large mass ratio, the planet experiences a negligible change in velocity; whereas, in the heliocentric reference frame, the velocity of the spacecraft can change in both direction and magnitude. ${ }^{[3]}$ In addition to gravity assisted flybys, performing impulsive maneuvers 
between planets, known as deep space maneuvers or DSM's, stands to reduce the total $\Delta \mathrm{V}$ even further.

In order to improve upon current methods for trajectory optimization, understanding the search space is critical. Simple trajectory designs consist of a single direct transfer between two planets, where the launch and arrival dates are the only optimization parameters. For cases like these, gradient based searches can quickly and accurately yield the global optimum solution. Furthermore, these solutions can be verified by visual inspection of overlaid 2-D contour plots known as porkchop plots. These plots are easily distinguishable by their periodic shapes which contain paired local minima. Typically porkchop plots associated with launches display the regions within a search space that contain the same C3 values, also known as the characteristic launch energy values. Orbital insertion porkchop plots, on the other hand, usually display the regions within a search space with the same $\Delta \mathrm{V}$. Even though the units between $\mathrm{C} 3$ and $\Delta \mathrm{V}$ differ, if no constraints are placed on the design, then the optimal solution exists near the minimum point obtained by superimposing the quantities of the launch and orbit insertion plots. These plots are advantageous to mission designers and the quantitative data from them is highly useful for improving the quality of stochastic methods. Unfortunately, the process to determine this data is often deterministic and can be computationally expensive.

COMET utilizes grid sampling to evaluate the search space. Here, each dimension of the search space is discretized into a finite set of points, and then meshed together to create a grid. For a given search space, the sampling frequency, or how far apart the discretized points are, directly correlates to the size of the grid and the quality of 
knowledge regarding the search space. COMET allows users to increase the sampling frequency, enabling the number of grid points to grow. This allows for fewer areas of the search space to be overlooked. Figure 1 shows an example of the porkchop plot for launch for a simple Earth to Venus transfer using a 7 day grid sampling frequency for both the launch date and arrival date dimensions produced by COMET.

Determining the initial and final velocities for the trajectories corresponding to each grid point is the most computationally expensive aspect involved in this processCOMET reduces these computations. This problem often referred to as Lambert's problem, named after Johann H. Lambert, uses Euler's formulas to solve for the initial and final velocities at two terminal positions when the time-of-flight and a direction of

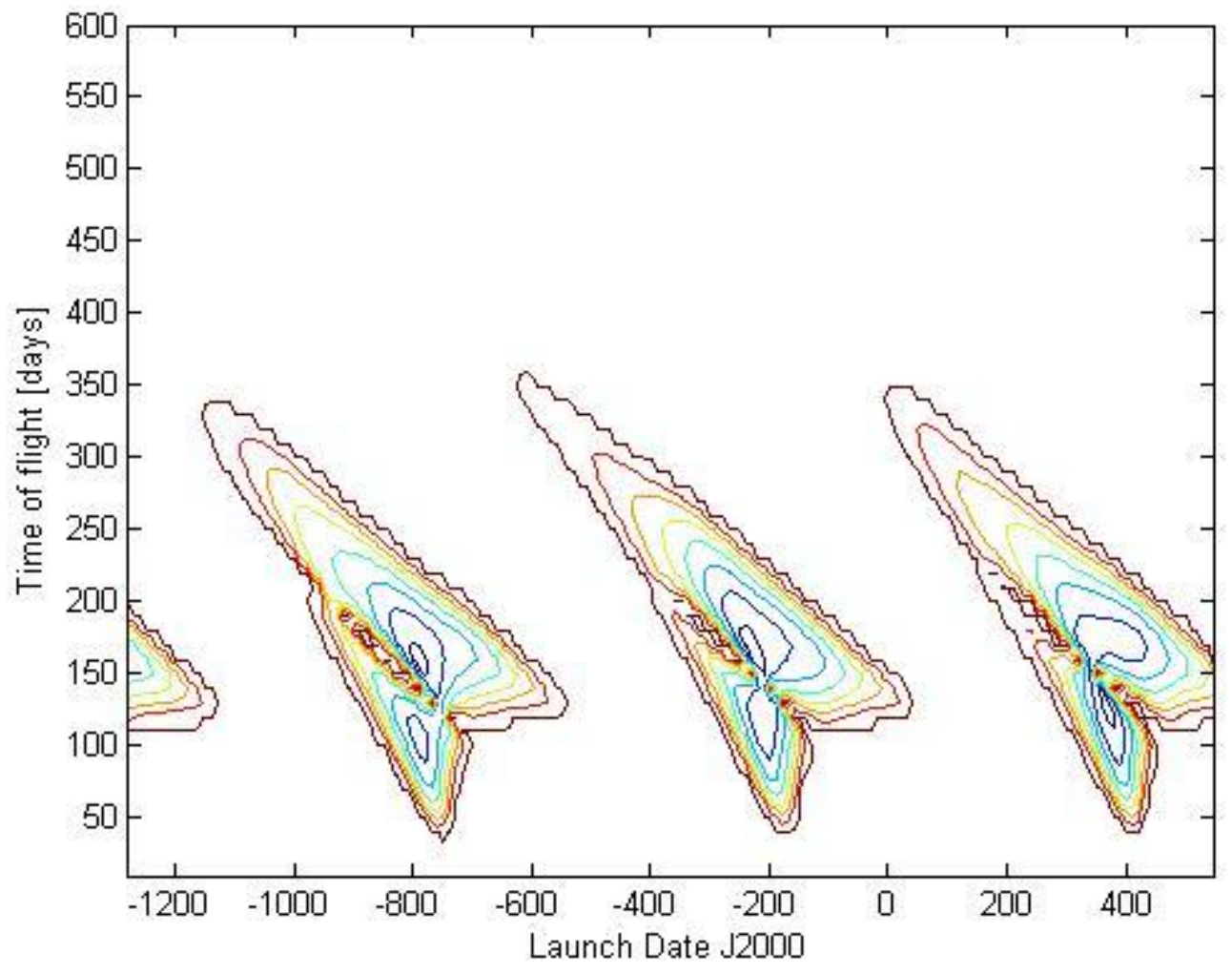

Figure 1: Earth to Venus launch porkchop plot 
motion between the positions are known. The problem, first addressed in 1744 by Leonhard Euler, has over the past two and a half centuries been examined through multiple approaches. ${ }^{[2,3,5,7,9,14]}$ Focus in the recent decades has shifted to determining more efficient ways to evaluate the problem using computational power. Currently, one of the best known methods to solve Lambert's problem uses a Gaussian approach and canonical units to mitigate computational error associated with significant digits. This algorithm was released by the European Space Agency and is publicly available on their website. ${ }^{[6]}$ Due to this algorithm's ability to compute over 100 solutions to Lambert's problem in under a second, COMET has adopted and modified the algorithm for its own use. Only allowing prograde trajectories removes a number of computational variable checks, decreasing COMET's computational time by up to $67 \%$. For the full Lambert's algorithm function used by COMET refer to appendix B.

In discussing trajectory efficiency the word optimal can be misleading. Trajectory design problems can quickly grow in dimension with the addition of every flyby and DSM. This increased complexity can quickly make determining the optimal trajectory infeasible. Consequently, the optimal solutions for most trajectory problems remain unknown. As a result the word "optimal" is often used to refer to the best known solution found so far, which is most likely "sub-optimal." Regardless, mission designers still need to produce acceptable solutions in a timely manner.

As a MATLAB based graphical user interface (GUI) program, COMET allows users to specify problem structures and visualize the results of a global search. While MATLAB lacks some of the efficiency of compiled languages, such as $\mathrm{C}$ and $\mathrm{C}++$, its wide use, accessibility, and affordability throughout the aerospace community makes it 
an ideal choice for mission designers. This allows COMET to adapt to personal needs while still maintaining a high level of robustness, efficiency, and accuracy for a wide range of trajectory design problems.

The European Space Agency's Advanced Concept Team established an open source online test bed known as the Global Trajectory Optimization and Problems database, GTOP, for the purpose of comparing different derivative-free methods across the operational research community. ${ }^{[1]}$ This provided much of the foundation and background for COMET. In addition to the multiple papers referenced, the Differential Evolution and Lambert's algorithms found in the open source code, named the Multiple Gravity Assist program, MGA, were adapted to form the primary components of the optimization algorithm used by COMET ${ }^{[6]}$ However, to restrict the search space and improve the quality of the initial population used by the Differential Evolution algorithm, COMET first prunes the search space through a cascading grid sampling algorithm. It additionally introduces the novel approach of decomposing elements relating to deep space maneuvers to improve the quality of individual population members. Finally, to the added benefit of the user, COMET produces various graphs and animations to convey the trajectory and information found for the optimal solution. 


\section{INTERPLANETARY TRAJECTORY PROBLEMS}

Interplanetary trajectory designs begin with determining the sequence of the planetary flybys and deep space maneuvers which will result in reaching the mission target. Since these sequences can be lengthy when written out in full, it is common practice to refer to just the first letter of the planet when describing a flyby sequence. For example, a mission that launches from Earth, followed by two Venus flybys, followed by an Earth flyby, then a Jupiter flyby, followed by a DSM, and finally inserting into orbit around Saturn, simplifies to EVVEJDS. Obviously, if either Mercury or Mars are in the sequence special care must be used to avoid confusion. All interplanetary trajectories consist of at least two planets, with the first typically being Earth. Simple cases, such as missions to Mars or Venus, usually are straightforward, as they require a sequence consisting of only two planets. However, for most other interplanetary missions the sequence may require serious forethought. The correct sequence of planets placed in the correct order is critical to determining the lowest $\Delta \mathrm{V}$ options available. While some programs are being developed to automatically assign or search for these sequences, this process is timely and for most programs like COMET, a predefined sequence is required.

To define a single trajectory, a date must be associated with each element in the sequence, whether it is a planet or deep space maneuver. Additionally, enough information is required to define the launch, the orbit of each flyby, and a general parking orbit at the final planet. If the trajectory contains DSM's, additional information about the positions where the DSM's occur is also required. In general, each of these parameters equates to a dimension in the optimization process. If the parameters are discretized into a finite set of values then the number of possible trajectories grows exponentially with 
each dimension. Therefore, it becomes pertinent to define the trajectory in as few dimensions as possible.

The dates associated with the launch, which range from the earliest possible to the latest acceptable launch dates, are used as the first parameter in the optimization. To make mathematical manipulations of these dates easier to understand, they typically are defined or converted into Julian dates or J2000 dates. A J2000 date refers to the difference in number of days from January 1, 2000 at 12:00:00 to the given date and time. Defining date ranges for the rest of the planets in the sequence can result in checking overlapping dates and infeasible transfer times. To mitigate these concerns ranges of acceptable times-of-flight are used instead. Similar to flybys, assigning a range of dates to the DSM's can be problematic. However, instead of using two times-of-flight to describe the trajectory legs before and after the DSM, the total time-of-flight for both legs is used in conjunction with a value $\alpha$ which defines the percentage of that time-of-flight when the DSM occurs. These $\alpha$ values typically range from $10 \%$ to $90 \%$.

To begin evaluating a trajectory the state vectors when the spacecraft arrives and leaves a planet or maneuver must be calculated. For a given trajectory where the launch date and all times-of-flight are defined, the state vectors for each planet can be calculated from ephemeris tables. This leaves only the positions of the DSM's and the parameters defining the flybys unknown. To solve for these, first all outbound and inbound velocity vectors for trajectory legs between two planets are determined by solving Lambert's problem. With the exception of the flyby directly before or after a DSM, from these velocities, the relative hyperbolic velocities for launch, orbit insertion, and the remaining flybys can be evaluated. For these remaining flybys, known as powered flybys, crossing 
the relative inbound and outbound velocity vectors will produce a vector normal, $\hat{n}$, to the hyperbolic flyby plane known as the B-plane. The eccentricity, e, and periapse radius, $r_{p}$ for these flybys can then be determined based off the change in the in-plane angle for the two relative velocity vectors known as the turn angle, $\delta$ and the relative inbound and outbound hyperbolic velocities, $\mathrm{V}_{\infty}{ }^{-}$and $\mathrm{V}_{\infty}{ }^{+}$respectfully, see Figure 2. This process can be seen in the following equations, where $\mathrm{V}_{\text {planet }}, \mathrm{V}^{-}$, and $\mathrm{V}^{+}$, are the helio-centric velocities of the flyby planet, and the inbound and outbound spacecraft, respectfully, and $\mu_{\text {planet }}$ represents the gravitational parameter of the flyby planet.

$$
\begin{gathered}
V_{\infty}^{-}=V^{-}-V_{\text {planet }}, v_{\infty}^{-}=\left\|V_{\infty}^{-}\right\| \\
V_{\infty}^{+}=V^{+}-V_{\text {planet }}, v_{\infty}^{+}=\left\|V_{\infty}^{+}\right\| \\
\hat{n}=V_{\infty}^{-} \times V_{\infty}^{+} \\
\delta=\cos ^{-1}\left(\frac{\left(V_{\infty}^{-} \cdot V_{\infty}^{+}\right)}{v_{\infty}^{-} * v_{\infty}^{+}}\right) \\
e=\frac{1}{\sin \left(\frac{\delta}{2}\right)} \\
r_{p}=\frac{(e-1) * \mu_{\text {planet }}}{v_{\infty}^{-}}
\end{gathered}
$$

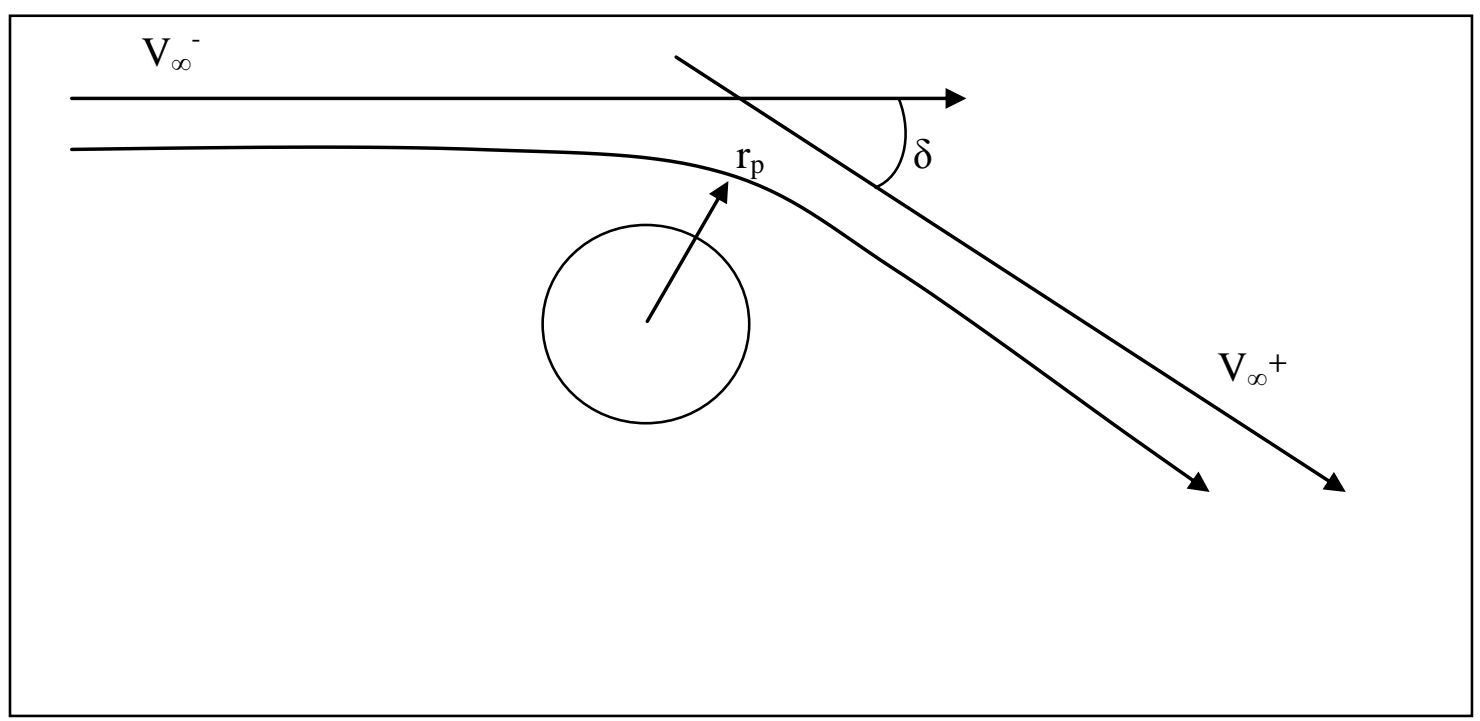

Figure 2: Illustration of flyby values $r_{p}$ and $\delta$ in B-plane 
For the flybys preceding DSM's, the outbound velocities are initially unknown. Consequently, $\mathrm{r}_{\mathrm{p}}$ and a value known as $\beta$, which defines the rotation of the B-plane about the inbound velocity vector, must be included as optimization parameters. When $\beta$ equals zero the B-plane contains the inbound velocity vector and is perpendicular to the position vector of the planet relative to the sun. Placing the plane initially perpendicular to the position vector ensures that the optimal $\beta$ values for dark-side flybys will be near $-\pi / 2$ rather than near $\pm \pi$, increasing the probability of converging to the optimal solution when using COMET's Differential Evolution global optimizer. With the $r_{p}$ and B-plane defined, the turn angle, $\delta$, can be backed out of the previous equations. The outbound velocity vector can then be determined using a quaternion rotation of the inbound velocity vector about the axis perpendicular to the $\beta=0$ plane by the angle $\delta$, followed by another quaternion rotation about the normalized original inbound velocity vector by the angle $\beta$-refer to the function DSM_DV.m for the full equations of rotation. With the outbound velocity established, the method of universal variables, which transforms the classical orbital elements into a set variables where time is the only independent variable, is used to propagate the spacecraft's position and velocity to the DSM. ${ }^{[14]}$ After the position of the DSM has been determined, Lambert's problem can again be solved to determine the remaining trajectory leg between the DSM and the next planet. The inbound velocity for the flyby after the DSM is now known and the remaining $r_{p}$ and Bplane can be determined in the same fashion as described above.

Since the complexity of a problem increases with each dimension, it is paramount to reduce the necessary dimensions needed to define the trajectory. The launch maneuver can be reduced to two dimensions by assuming the maneuver to be a direct injection into 
the transfer orbit. This is possible since the state vectors of the launch planet and the hyperbolic escape velocity vector sufficiently define the launch, making the launch date and time-of-flight to the following planet the only optimization parameters needed for launch. Using the previous equations, powered flybys can be defined using only three dimensions: the date the spacecraft left the preceding planet, the time-of-flight to reach the flyby planet, and the time-of-fight to reach the following planet. DSM's require an additional three dimensions to be considered fully defined: $\alpha, r_{p}$, and $\beta$. Finally, for the orbit insertion phase, a general parking orbit must be defined. A simple parking orbit consists of a periapse radius, $r_{p}$, and an eccentricity, e. Based on these two values, those listed in the previous equations, a general periapse velocity can be determined for both the parking orbit and the inbound hyperbolic orbit using the equations below.

$$
\begin{gathered}
a_{\text {hyp }}=-\frac{\mu_{\text {planet }}}{\left(v_{\infty}^{-}\right)^{2}} \\
v_{\text {hyp }}=\sqrt{\mu_{\text {planet }} *\left(\frac{2}{r_{p}}-\frac{1}{a_{\text {hyp }}}\right)} \\
a=\frac{r_{p}}{1-e} \\
v_{p}=\sqrt{\mu_{\text {planet }} *\left(\frac{2}{r_{p}}-\frac{1}{a}\right)}
\end{gathered}
$$

The orbit insertion can then be evaluated by comparing the parking orbits velocity with the inbound hyperbolic velocity at the same altitude. Since the periapse radius and eccentricity can be held constant, the only optimization parameter necessary for the orbit insertion is again the time-of-flight previously mentioned. In summary, the 
dimensionality of the optimization search is equal to the number of planets plus three times the number of DSM's.

While this may provide a sufficient amount of information in order to perform the optimization process, to meet additional mission designer needs, COMET requires the definition of a few more parameters. To avoid the possibility of colliding with a planet or interacting with its atmosphere, a minimum allowable $r_{p}$ is assigned to each flyby. Additionally, for the pruning process, COMET requires a maximum $\Delta \mathrm{V}$ for each element in the sequence, as well as, the sampling frequency rate for each range-of-dates or timesof-flight dimension. For the optimization process, a weighting for each maneuver, $\mathrm{w}_{\mathrm{i}}$, allows the user to define their personal preference on which maneuvers should be penalized more for having a larger $\Delta \mathrm{V}$. Finally, an overall weighting, W, which allows the user to place more or less emphasis on mission time compared to total $\Delta \mathrm{V}$ is also required. By default, all $\mathrm{w}_{\mathrm{i}}$ 's are set to 1 and $\mathrm{W}$ is set to 0 , making each maneuver equally important and placing full emphasis on $\Delta \mathrm{V}$ while ignoring mission time. The effects of each of these weightings can be seen in the cost model at the end of this section.

The effectiveness of each trajectory is based on the $\Delta \mathrm{V}$ 's for each individual maneuver. In general, a $\Delta \mathrm{V}$ can be calculated as the magnitude of the difference in velocities prior to and after a maneuver. However, in order to make these calculations, all the velocities must be known in advance. Ephemeris data can be used to calculate the state vectors for the planets and dates in the sequence. Using these position vectors and given times-of-flight, Lambert's problem can solve for any legs of the trajectory between two planets. For DSM's, the outbound velocities of the preceding flybys are calculated using the given $r_{p}$ and $\beta$ values. As stated above, the position of the DSM's are found 
through the propagation of the outbound velocities from preceding flybys. With the positions known, Lambert's problem can solve for the remaining legs of trajectory.

Launch is assumed to be a direct injection into the transfer orbit and the velocity before the maneuver can be assumed to be the same as the velocity of the planet. To account for the gravitational assistance of a flyby, additional calculations are necessary. If the required $r_{p}$ for the flyby is greater than minimum $r_{p}$, then the $\Delta V$ is simply the difference in magnitudes of the velocities before and after the flyby, relative to the velocity of the planet. If however, the turn angle of the flyby requires a closer approach than the minimum $r_{p}$, then COMET calculates a new outbound velocity for the flyby with the minimum $r_{p}$ using a similar approach to the flyby calculations preceding a DSM. With the B-plane already established the outbound velocity results from a quaternion rotation of the inbound velocity about the normalized $\hat{n}$ vector. Refer to the function flyby_dv.m for the full equations of rotation. The difference between this new velocity and the desired outbound velocity serves as the $\Delta \mathrm{V}$ for the flyby. The $\Delta \mathrm{V}$ for DSM's is simply the change in velocity from before the DSM compared to after. Finally, the $\Delta \mathrm{V}$ for orbit insertion at the last planet is calculated as the magnitude of the difference in the transfer orbit's hyperbolic velocity at the given $r_{p}$ of the parking orbit and the velocity of the parking orbit at that same position.

While a crude model maybe sufficient for some cases, COMET attempts to minimize the actual propellant necessary to perform these maneuvers and to accommodate for the mission designers' needs. In this simplistic model, the cost of a trajectory consists only of the summation of all $\Delta \mathrm{V}$ 's in the sequence. To do so, a combination of penalty factors, as well as, the previously mentioned user defined 
preferential weighting factors are applied to the cost equation. Trajectories are penalized for having large individual $\Delta \mathrm{V}$ 's and for having a larger portion of the $\Delta \mathrm{V}$ 's occurring later in the trajectory. The cost model used by COMET can be seen in the equation below, where $T_{\text {total }}$ represents the total mission time and $T_{i}$ represents the time since the mission epoch when the $\mathrm{i}^{\text {th }}$ maneuver occurs.

$$
J=(1-W) T_{\text {total }}+W \sum_{\mathrm{i}=1}^{\mathrm{n}} \mathrm{w}_{\mathrm{i}}\left(\frac{\Delta \mathrm{V}_{\mathrm{i}}}{\Delta \mathrm{V}_{\mathrm{i}, \text { max }}}\right)^{2}\left(\frac{\mathrm{T}_{\mathrm{i}}}{\mathrm{T}_{\text {total }}}\right)
$$




\section{SEARCH SPACE PRUNING}

Stochastic methods, like the ones used by COMET to initialize the populations in Differential Evolution algorithms, establish parameter values by generating random values within a given search space. While advantageous in avoiding periodic behaviors and preventing stagnation when applied to iterative processes, these methods suffer from proportional inefficiency when it comes to trajectory optimization problems. The probability of a randomly generated point being a feasible solution is equal to the ratio of feasible to infeasible search space. As can be seen in the Figure 3 below, for a given 2-D problem where the feasible limit on C3 energy is set to $100 \mathrm{~km}^{2} / \mathrm{s}^{2}$ roughly only $20 \%$ of the total search space is feasible. As a result randomly generating 10 points within the given search space only captures two feasible solutions.

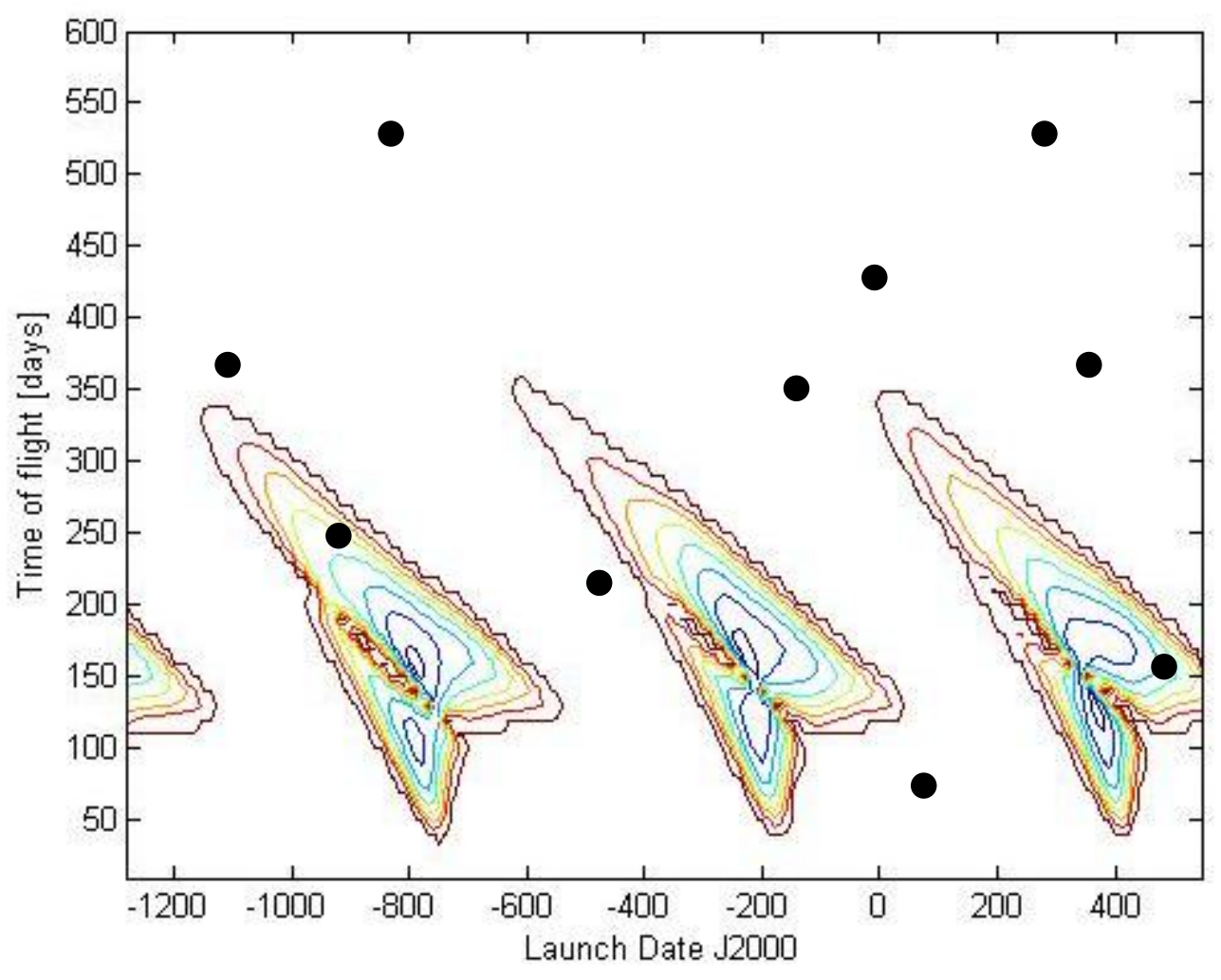

Figure 3: Random generation of points in a search space before pruning 
By first pruning the search space, it effectively restricts the generation of the random values to fall within only valid ranges. In the example above $80 \%$ of the points generated fell in infeasible regions. In addition to these points providing no improvement to the population, they cost the optimization function time and memory to compute. The same example is shown in Figure 4 below with the search space pruned. Note how 100\% of the randomly generated points now fall in feasible regions, greatly improving the quality of the population.

Determining what portions of the search space are valid reduces the order of the overall problem and drastically improves the computational run time. COMET utilizes a cascading grid sampling algorithm to achieve this goal. This algorithm partitions the $\mathrm{n}$ dimensional problem into a series of two-dimensional and three-dimensional problem

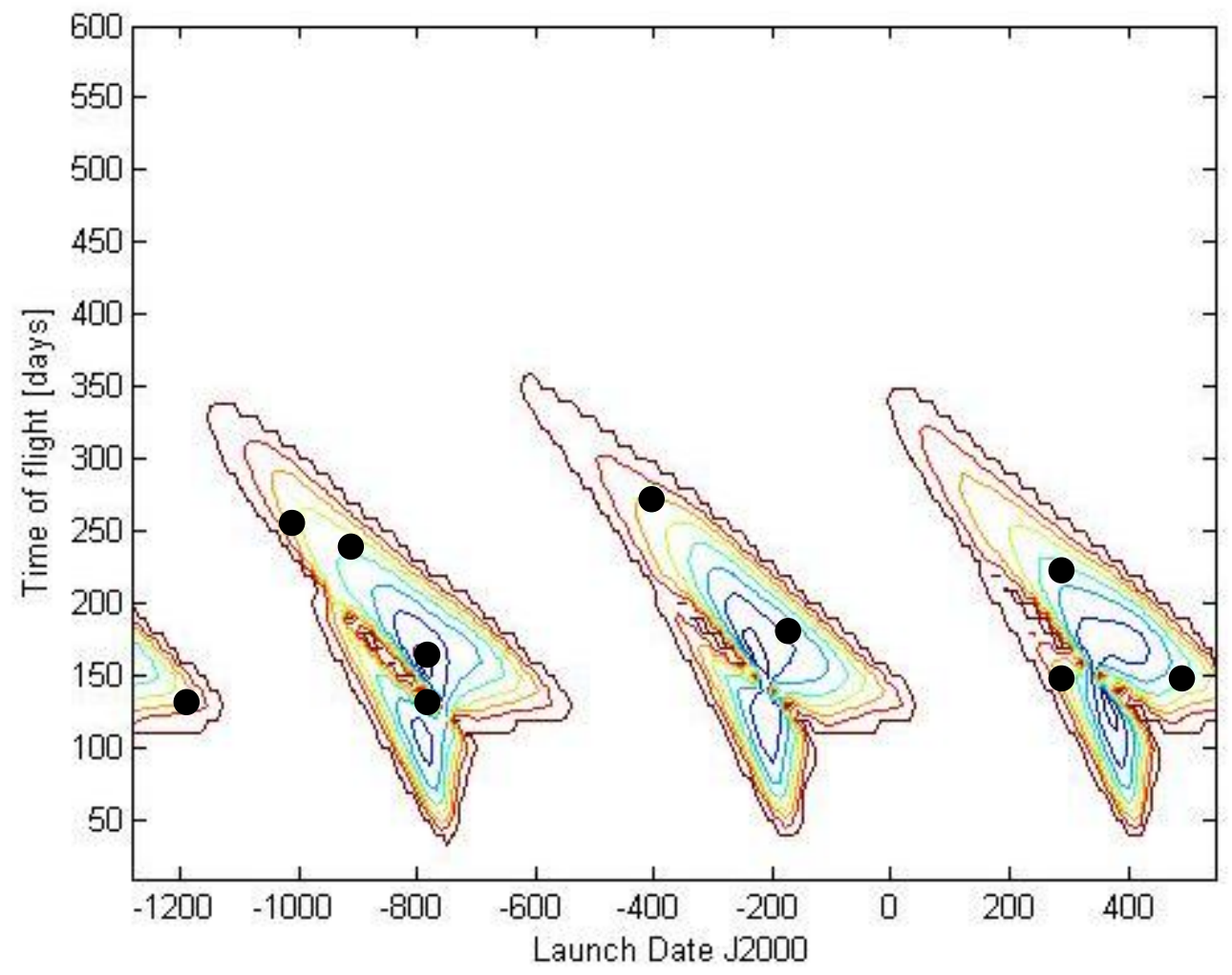

Figure 4: Random generation of points in a search space after pruning 
sets. For a given sequence of planets and deep space maneuvers the infeasibility of a particular launch trajectory is dependent only on the launch date and the arrival date at the next planet. This allows the algorithm to view this as a two-dimensional problem. Similarly, the practicality of an orbit insertion maneuver or rendezvous with the final planet depends solely on the date of the arrival and the date of the preceding flyby, again allowing the algorithm to view this as a two-dimensional problem set. The infeasibility of flybys adds the complexity of an additional dimension, since it is defined by the date of the flyby and the dates when the spacecraft left the preceding planet and the arrival at the planet following the flyby, thus making it a three-dimensional problem set. The parameters for DSM's are not pruned currently.

To check the validity of a launch, the date of the launch and the date when the satellite reaches the following planet are used to solve Lambert's problem. Based on the difference between the outbound velocity at the launch planet and the velocity of the planet itself, the given transfer orbit can be ruled either acceptable or not. Similarly, based on the date when the spacecraft left the planet before the orbit insertion, and the date of the orbit insertion the inbound velocity can be used to calculate the hyperbolic velocity at periapse. If the velocity is within an acceptable range of the parking orbit's periapse velocity, defined by the user, the transfer is considered valid. Since these validity checks only require two variables the results can be displayed as contour plots or porkchop plots like the one in Figure 1. If a DSM precedes the orbit insertion, then all dates for the insertion are considered valid.

A flyby requires the use of three dates: the date when the spacecraft left the previous planet in the sequence, the date of the flyby, and the date when the spacecraft 
reaches the next planet in the sequence. These dates will be denoted as $D_{n-1}, D_{n}$, and $D_{n+1}$, respectfully. Based on these dates, the necessary inbound and outbound velocities at the flyby can be calculated. The validity of a flyby is determined through a three part check. First, if the difference in magnitudes of the inbound and outbound relative velocities exceeds the allowable limit, again defined by the user, then the flyby is deemed invalid. If it passes the first test, then the necessary $r_{p}$ to generate the needed turn angle is determined. If the $r_{p}$ is greater than the minimum allowable $r_{p}$, then the flyby is valid. If not, then through the use of quaternion rotations the outbound velocity with the minimum $r_{p}$ is evaluated. If the change in velocity between this new outbound velocity and the desired velocity are acceptable, then the flyby is saved as a valid solution.

After all possible valid date combinations for a flyby have been evaluated, the pruning program evaluates the next flyby in the sequence. At this point all valid $\left[D_{n} D_{n+1}\right]$ combination of dates of the preceding flyby become the $\left[\mathrm{D}_{n-1} \mathrm{D}_{\mathrm{n}}\right]$ combination of dates used to evaluate the current flyby. However, since the velocity at $\mathrm{D}_{\mathrm{n}+1}$ only depends on $D_{n}$, there may exist multiple $D_{n-1}$ dates that yield the same $\left[D_{n} D_{n+1}\right]$ combination of dates. In the next leg of the trajectory, this will result in redundant evaluations of the same $\left[D_{n-1}\right.$ $\mathrm{D}_{\mathrm{n}}$ ] date combinations. To improve efficiency, these redundancies are removed, but as will be discussed below, it remains necessary to retain the information for all $\left[D_{n} D_{n+1}\right]$ combinations, in order to backtrack and prune trajectory branches that reach a dead end.

The complexity of this backtracking system revolves solely around the storage and updating of the indices associated with each of the dates. An array of $\mathrm{D}_{\mathrm{n}+1}$ dates and the corresponding planetary state vectors are created to accommodate any $\left[D_{n-1} D_{n}\right]$ date combinations. The earliest date in the $\mathrm{D}_{\mathrm{n}+1}$ array is established by adding the minimum 
time-of-flight to the earliest $D_{n}$ date. Similarly, the upper end of the $D_{n+1}$ array is established by adding the maximum time-of-flight to the latest $\mathrm{D}_{\mathrm{n}}$ date. The intermediate $\mathrm{D}_{\mathrm{n}+1}$ dates are determined based on the ratio of the grid sampling frequencies between the previous leg and the current leg of the trajectory. For example, as seen in Figure 5, when $D_{n}$ is sampled every 14 days and $D_{n+1}$ is sampled every 21 days, the array of $D_{n+1}$ dates must be spaced by 7 days. As in this example, this value maybe less than the intended grid sampling frequency. Consequently, a value has to also be defined to specify how many indices should be skipped over to increment the correct number of days. For the previous example, to sample every 21 days the program must skip over two grid points. Table 1 shows the corresponding spacing of dates and skipping factor for each ratio of sampling frequencies. The example above corresponds to the ratio $21: 14$, shown in the eighth column.

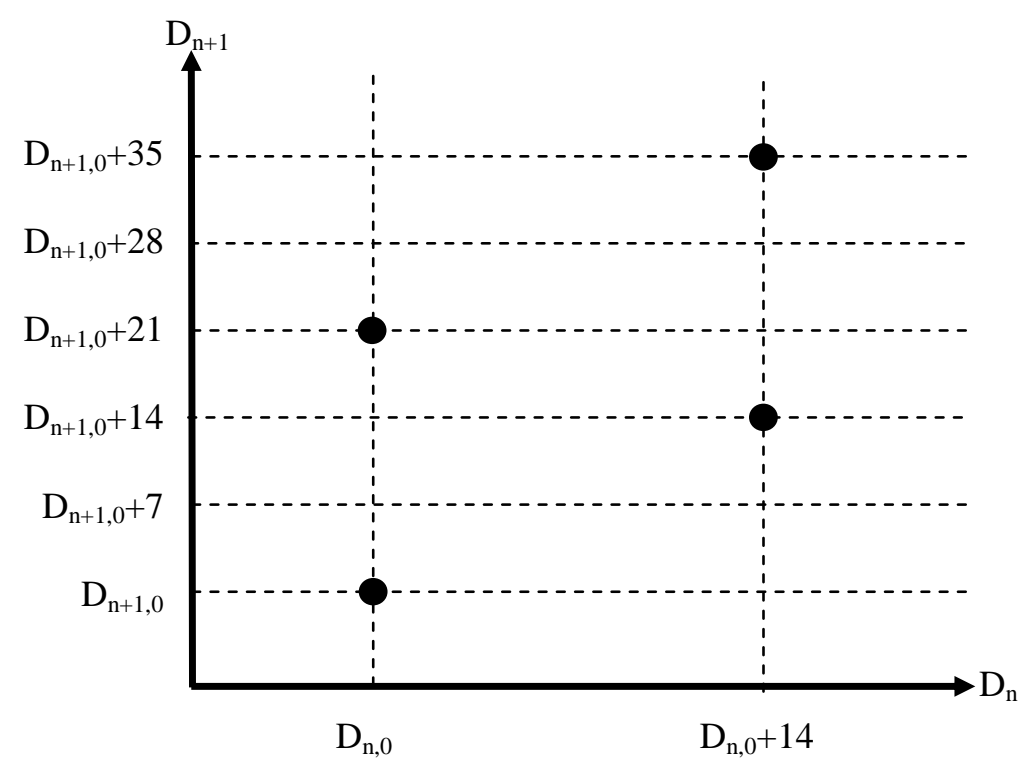

Figure 5: Illustration of unequal grid spacing on date indexing 
Table 1: Date spacing and index skipping values to unequal grid spacing ratios

\begin{tabular}{|l|l|l|l|l|l|l|l|l|l|l|l|l|l|}
\hline Ratio & $1: 7$ & $1: 14$ & $1: 21$ & $7: 14$ & $7: 21$ & $14: 21$ & $21: 14$ & $21: 7$ & $21: 1$ & $14: 7$ & $14: 1$ & $7: 1$ & 1 \\
\hline Spacing & 1 & 1 & 1 & 7 & 7 & 7 & 7 & 7 & 1 & 7 & 1 & 1 & Equal \\
\hline Skip & 6 & 13 & 20 & 1 & 2 & 2 & 2 & 0 & 0 & 0 & 0 & 0 & 0 \\
\hline
\end{tabular}

External indices, $i$ and $j$, are used to step through the valid $D_{n-1}$ and $D_{n}$ dates, respectfully. A third external index, $k$, is used to step through all possible $D_{n+1}$ dates within the acceptable time-of-flight range from $D_{n}$. When a valid $D_{n+1}$ date is found, two internal indices, $\mathrm{J}$ and $\mathrm{K}$, are used to store necessary information for the valid solution including: Julian dates, planetary states, velocities, and a backtracking variable containing the corresponding $\mathrm{i}$ and $\mathrm{j}$ indices for the current solution. Additionally, flags are created to indicate that at least one valid solution for the $\mathrm{i}$ and $\mathrm{j}$ coordinates exists. After each valid solution is found, the $\mathrm{K}$ index is incremented by one, and a flag is created to increment $\mathrm{J}$ after all $\mathrm{D}_{\mathrm{n}+1}$ dates have been checked. When $\mathrm{J}$ is incremented, the $K$ index is reset to 1 . If the same $\left[D_{n} D_{n+1}\right]$ date combination is found later, the $i$ and $j$ indices at that time are appended to the backtracking variable, but $\mathrm{J}$ and $\mathrm{K}$ do not increment. If the flag indicating that $\mathrm{j}$ has at least one valid solution is never created, then no $D_{n+1}$ date is valid for the given $\left[D_{n-1} D_{n}\right]$ date combination and this leg is flagged to be removed. Furthermore, if the flag indicating i has a valid solution is never created, then there exists no valid trajectory with the current $D_{n-1}$ and all trajectories leading up to $D_{n-1}$ are flagged to be removed. A visual representation can be obtained through a volume visualization process where the valid solutions within the search space are connect and enclosed with a surface. An example of this can be seen in Figure 6, which illustrates the valid search space regions for the second Venus flyby in the EVVEJDS problem. 


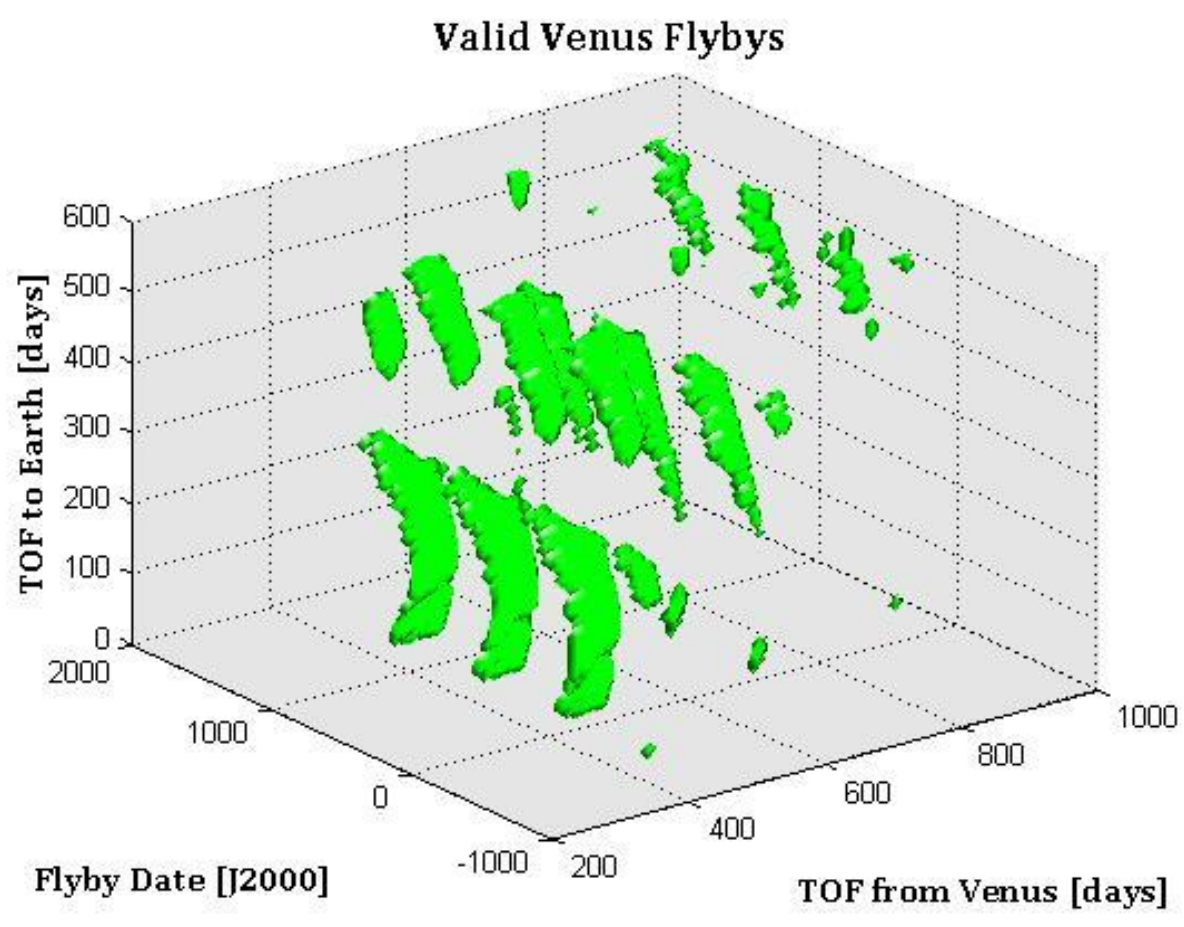

Figure 6: Visualization of valid search space regions for a flyby

Once COMET has checked all possible $\left[\begin{array}{lll}D_{n-1} & D_{n} & D_{n+1}\end{array}\right]$ date combinations for a given flyby, any $\left[D_{n-1} D_{n}\right]$ trajectory legs that were flagged to be removed are passed into its pruning algorithm. Since the indices $i$ and $j$ were incremented regardless of whether or not a valid solution was found for the $\left[D_{n-1} D_{n}\right]$ dates associated with them, all subsequent backtracking indices have to be adjusted to compensate for their removal. Prior to the removal of any trajectory legs, a temporary matrix of the current backtracking indices is created. To accomplish the task of removing the invalid trajectory, the invalid $\mathrm{i}$ and $\mathrm{j}$ indices are stepped through in reverse order. This ensures that only $\mathrm{i}$ and $\mathrm{j}$ indices greater than those being removed are adjusted. When a $\mathrm{j}$ index associated with a specific $\mathrm{i}$ index needs to be removed, the information related to this trajectory leg is deleted. Next, any backtracking $\mathrm{j}$ indices with the same $\mathrm{i}$ index and a value greater than that of the $\mathrm{j}$ being removed are reduced by one. In the events where all $\mathrm{j}$ indices for a given $\mathrm{i}$ index are flagged to be removed, all backtracking $\mathrm{i}$ indices with values greater than the $\mathrm{i}$ being 
removed are reduced by one. Additionally, when this happens it essentially implies that there exists no valid trajectory using the $\mathrm{D}_{\mathrm{n}-1}$ date associated with that $\mathrm{i}$ index. This means that any previous flyby validated using that date is now also invalid. Since the backtracking indices have been adjusted, the previous indices are pulled from the temporary matrix made at the start of the algorithm. After all invalid trajectory legs have been pruned for the current flyby, all the newly found invalid trajectory legs for the previous flyby are recursively passed into the pruning algorithm. This process continues until no further invalid trajectory legs are found or the process reaches the launch conditions. Figure 7 depicts the pruning of invalid trajectories and the shifting of indices. After all flybys, launch, and orbit insertion maneuvers are evaluated, all valid dates for each maneuver are compiled. Based on these dates, new bounds for the launch dates and times-of-flight optimization parameters can be determined. COMET passes these bounds and the newly pruned search space into the Differential Evolution algorithm to begin the

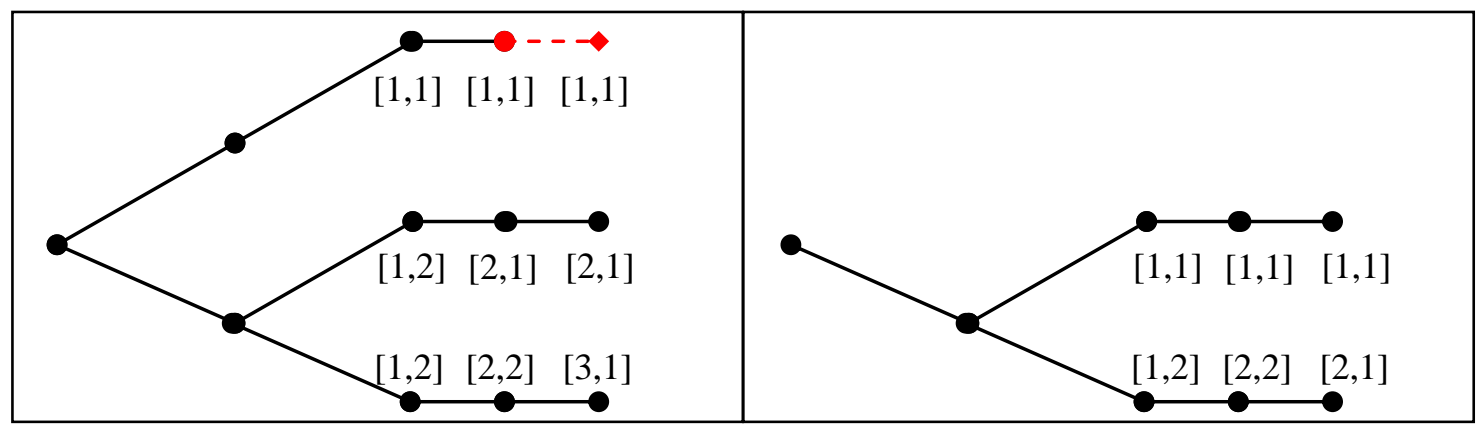

Figure 7: Illustration of pruning invalid trajectory legs

optimization process. 


\section{DIFFERENTIAL EVOLUTION}

First appearing in a technical report in 1995, Differential Evolution, DE, was

developed by Kenneth Price and Rainer Storn at UC Berkeley. ${ }^{[10]}$ Their ambitions were to create a highly adaptable global optimization evolutionary algorithm that could be applied to a wide range of problems. Shortly after its release, it took third place in the International Contest on Evolutionary Optimization, losing to two algorithms that had been specifically designed to the contest's problems. However, when applied to problems such as trajectory optimization, Differential Evolution showed significantly better results. In a paper released by the European Space Agency, Differential Evolution was compared to the following global optimization algorithms: DIRECT-Divided Rectangles, Multilevel Coordinate Search (MCS), Probabilistic Global Search Lausanne (PGSL), Cross Entropy (CE), Genetic Algorithm (GA), Particle Swarm Optimization (PSO), and Multiple Particle Swarm Optimization (MPSO) ${ }^{[8]}$ Fourteen functions each with known global optimum value and each with a known issue common to confuse global optimizers were used as the test bed. While MCS and MPSO both in general obtained strong results, DE showed equally successful results and was notably the most robust. On top of efficiency and robustness, the straight forward nature of Differential Evolution makes it easy to adapt to any optimization problem and the ideal choice for the global optimization algorithm for COMET.

One of the first parameters COMET considers is the size and selection of the initial population. In general, it is recommended to use a population size ten times the number of dimensions in the problem. For the given trajectory problem, EVVEJDS, a population of 70 members would be considered suitable. If the search space for the 
problem has not been pruned, then each parameter is assigned a random value from between the upper and lower bounds for that parameter. If, however, the search space has been pruned, then the initial population will be greatly improved in quality. To define this initial population, the population members and subsequent optimization parameters are stepped through one at a time. Starting with the launch date for the first population member, a random number between 0 and 1 is multiplied by the total number of valid launch dates and then rounded up to the nearest integer. This serves as the i index for the launch date. Based on this index the launch date is retrieved and a new random number between 0 and 1 is multiplied by the total number of valid flyby dates for that given launch date. Again this value is rounded up and serves as the $\mathrm{j}$ index. After determining the corresponding time-of-flight for the given [i j] index pair, a search is performed to find the i index for that flyby with the same index pair stored in its backtracking variable. Once found, the date associated with this flyby should be the same as the date determined by adding the time-of-flight to the launch date. To determine the next time-of-flight parameter, a new random variable between 0 and 1 is multiplied by the number of valid trajectory branches leading away from the flyby with the given dates. This same process is repeatedly applied until all times-of-flight have been established. Since the parameters for any deep space maneuvers were not pruned, a random value between the upper and lower bounds for those parameters is assigned to each. Once all parameters have been defined for the first population member, these procedures are repeated for every member in the population, checking that no two population members chose the exact same parameters for launch dates and times-of-flight. With the initial population established the computation of the algorithm can begin. 
There are a variety of different forms of DE and the following will explain the procedures for the most common form of DE known as "DE/rand/1." It may be useful to refer to the flowchart in Figure 8 while reading this section. Regardless of which form of $\mathrm{DE}$ is used, the algorithm starts like most genetic and evolutionary algorithms by establishing a population of members through stochastic methods, see Figure 9. The optimization parameters values for each member of the population are randomly selected from within a set of upper and lower bounds and follow a uniform distribution. Based on the cost model for the function being optimized, the cost of each population member is found. At this point the various forms of $\mathrm{DE}$ begin to diverge in procedure. For the common form, "DE/rand/1," one of the population members is randomly selected as the base member. Two more population members that differ from the base member and each other are randomly selected. A difference vector is formed by subtracting these two members from each other, see Figure 10. Superposition is used to combine the base member and the difference vector which has been multiplied by a scaling factor, F, to form the mutation member u, see Figure 11. This mutation member is compared to the first member in the population. If the mutation member shows an improvement in the cost function, then it replaces that member in the next population, see Figure 12. If, however, the original member possesses a better cost than the mutation, then the mutation is ignored and the original member is carried into the next population, see Figure 13. The process repeats until every member in the original population has been compared to a mutation member. 


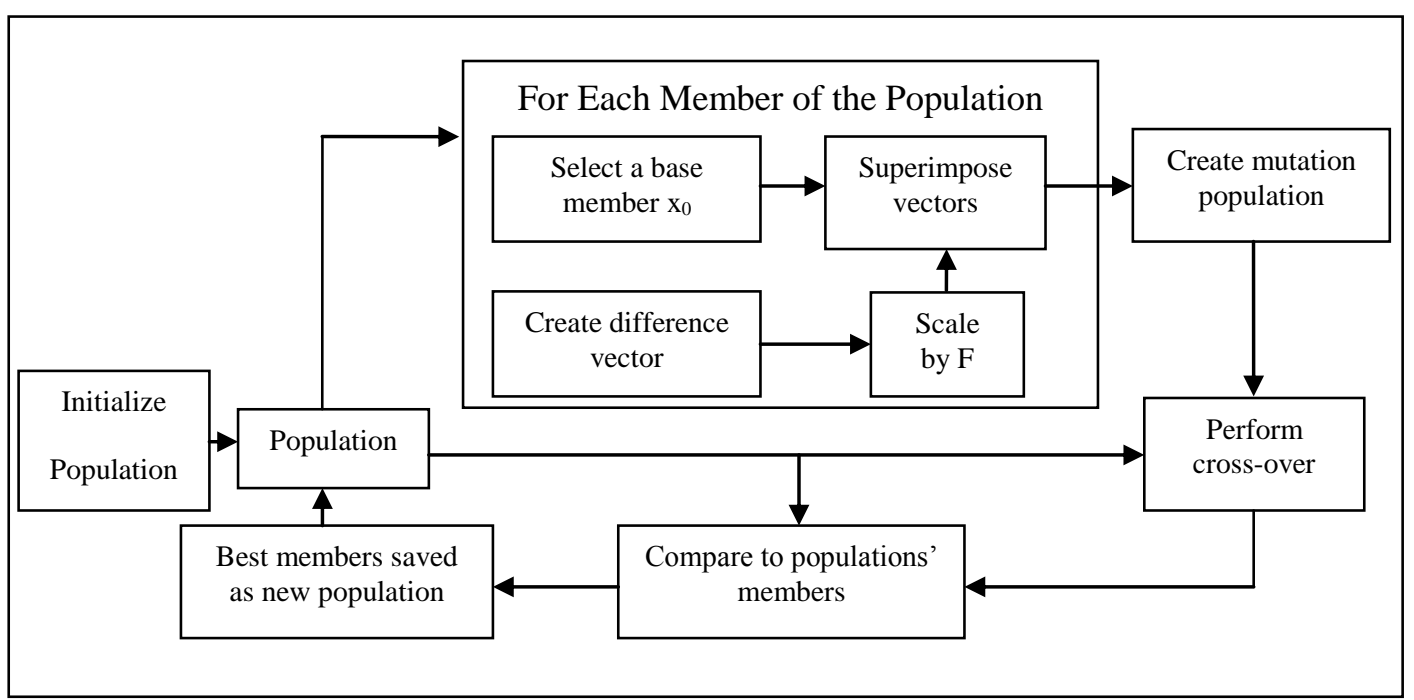

Figure 8: Differential evolution process

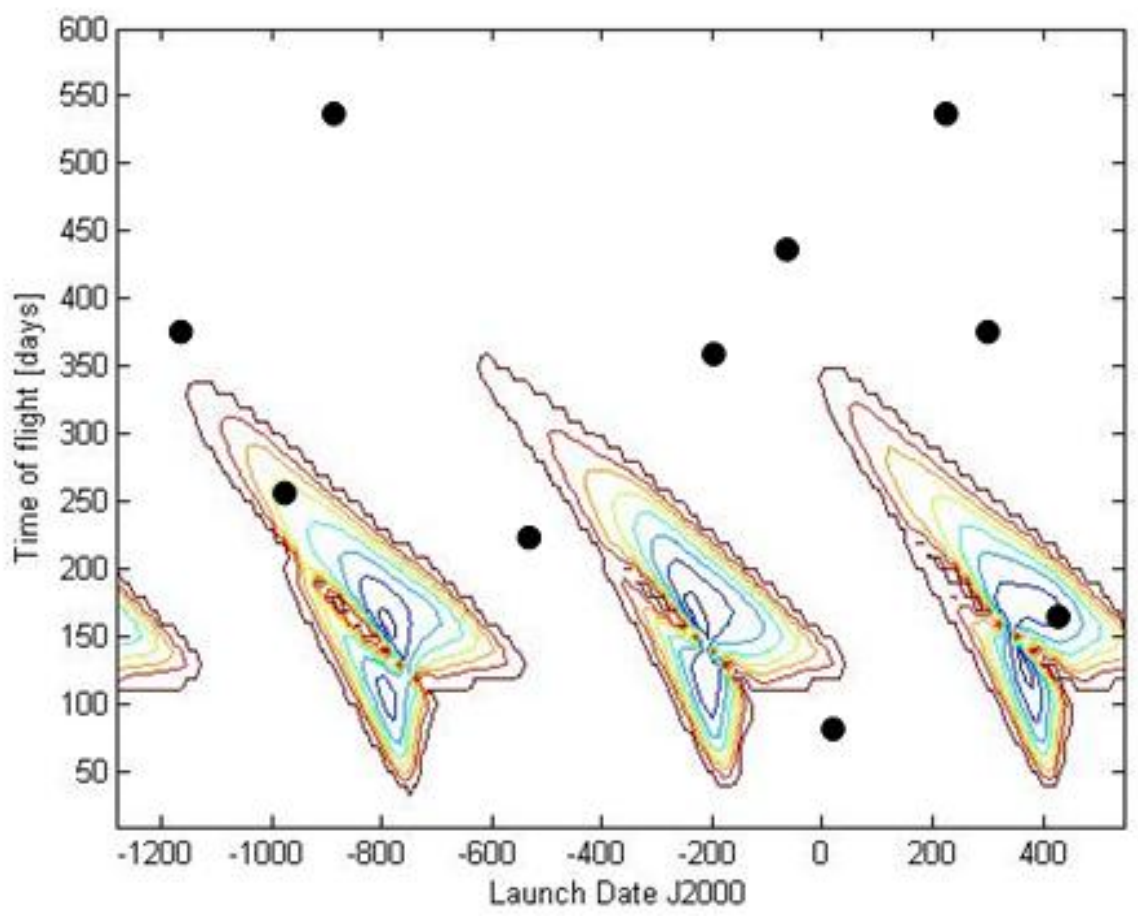

Figure 9: Initial population members for Differential Evolution 


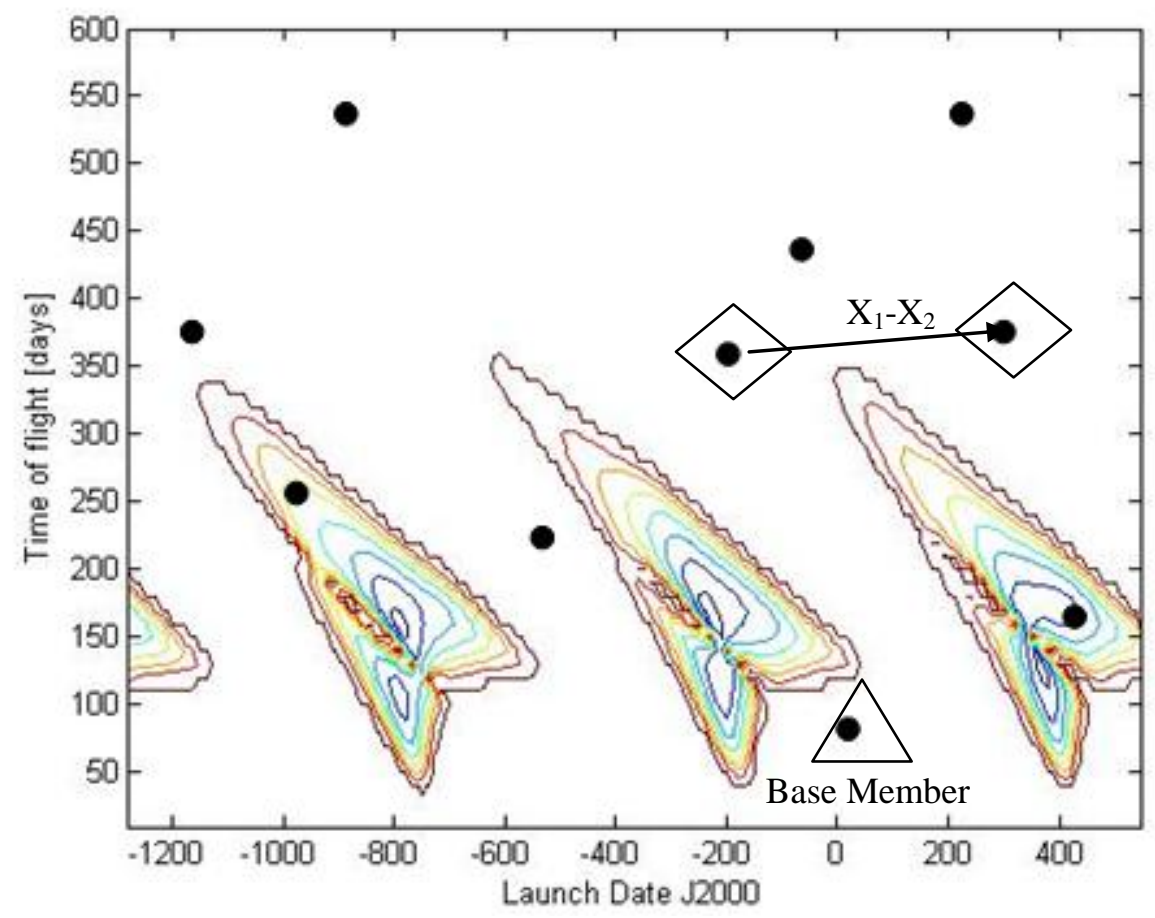

Figure 10: Selection of base member and difference vector

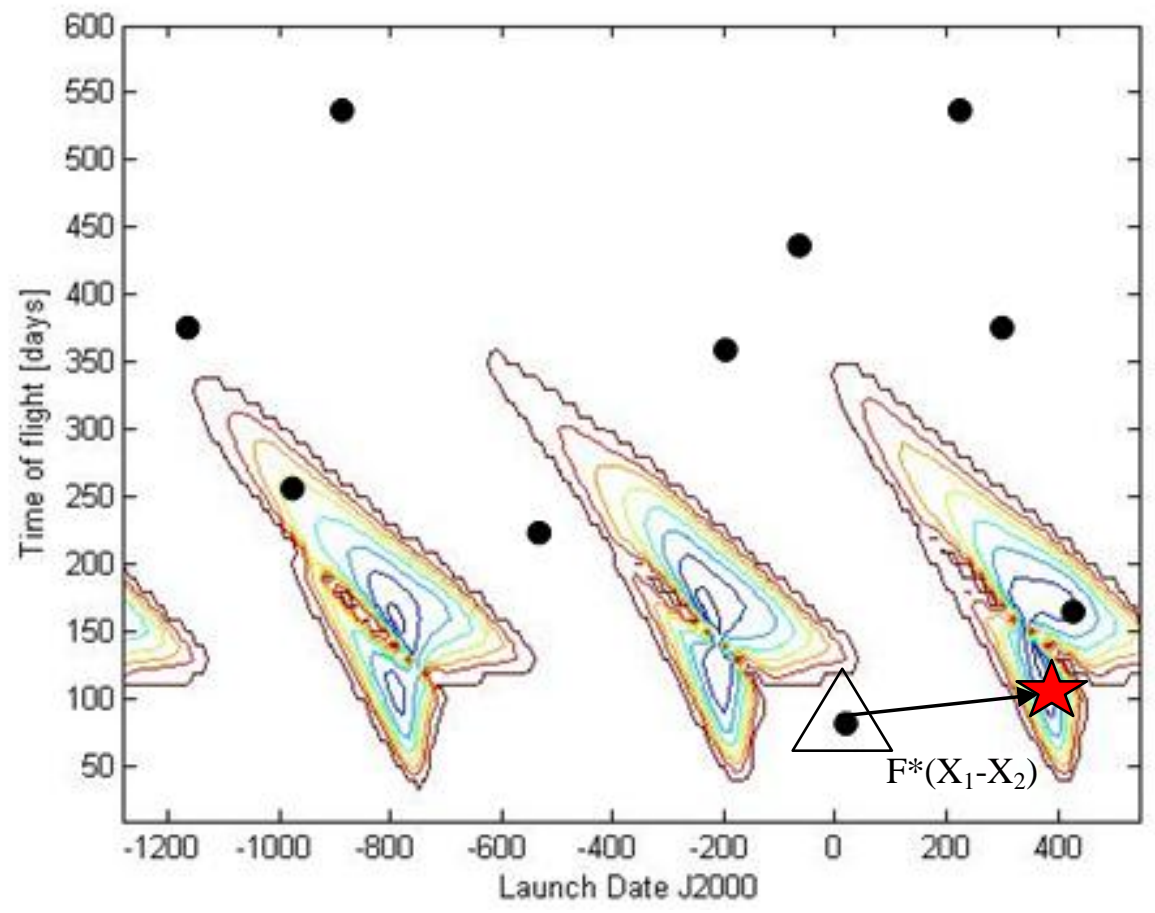

Figure 11: Generation of mutation member

$$
\mathbf{u}_{1}=\operatorname{Base}+F^{*}\left(\mathbf{X}_{1}-\mathbf{X}_{2}\right)
$$




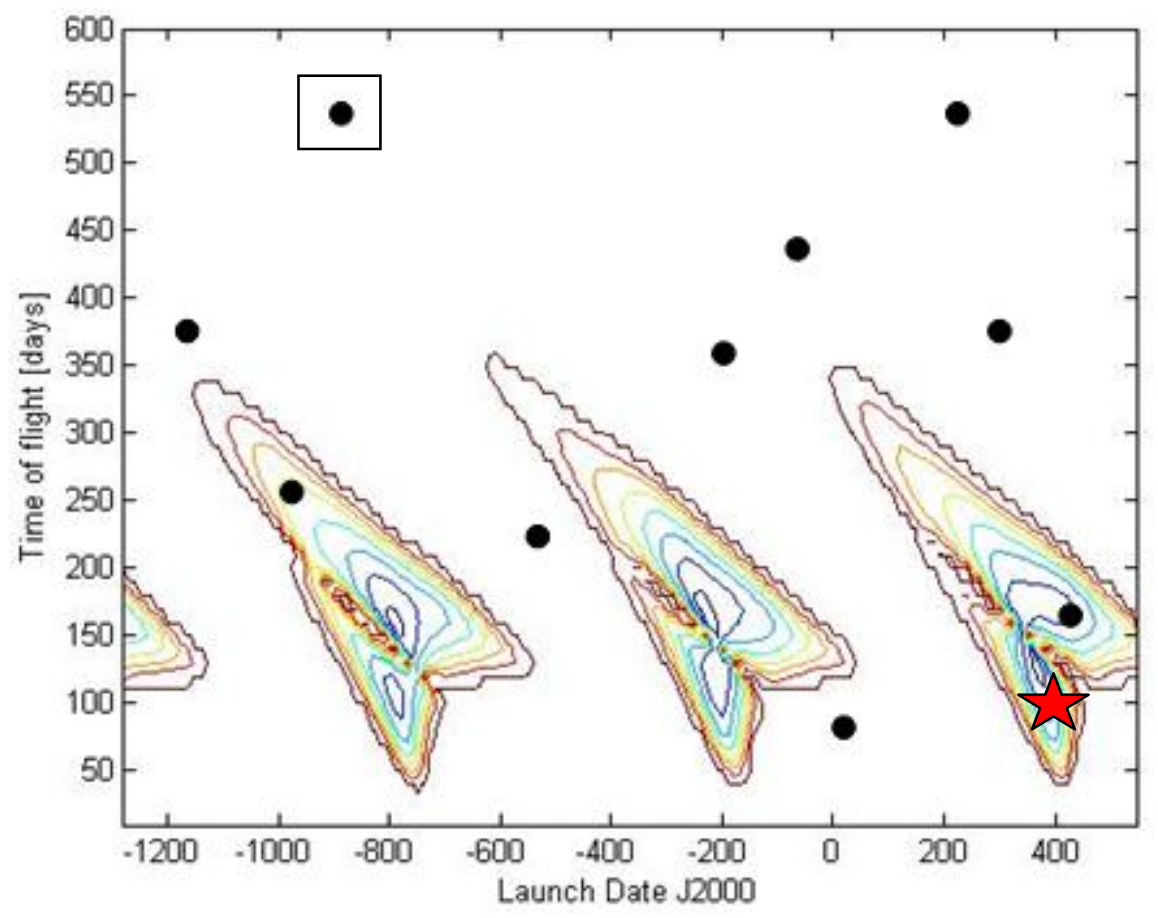

Figure 12: Selection process when mutation replaces original population member

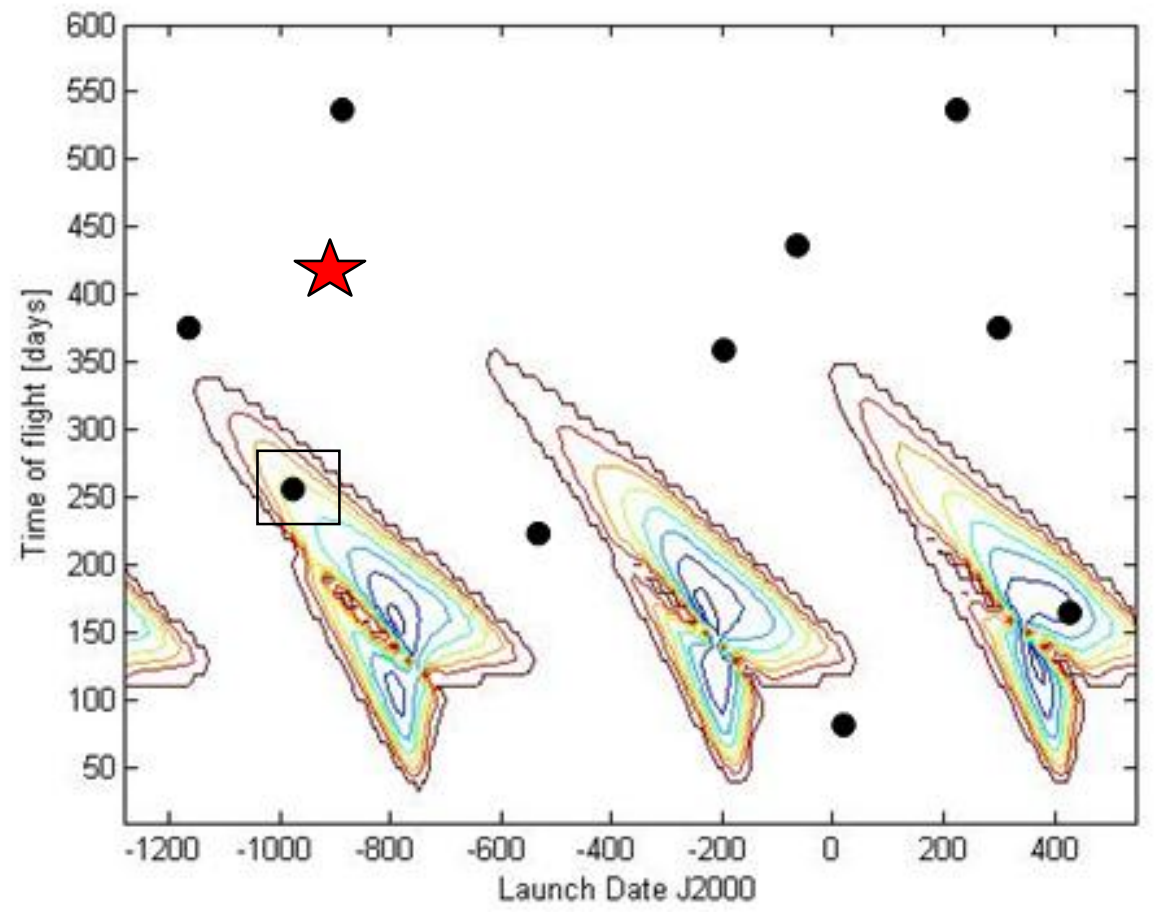

Figure 13: Selection process when original population member does not get replaced by mutation 
The mutation scaling factor, F, plays a critical role in the Differential Evolution process. While F can be any positive value, when it exceeds 1 , convergence can take longer and the produced result holds less reliability than had $\mathrm{F}$ been less than $1 .^{[10]} \mathrm{A}$ value of exactly 1 for $\mathrm{F}$ reduces the possible mutations by half, since any combination of three points can create the same mutation member, $X_{1}-\left(X_{2}+X_{3}\right)=X_{3}-\left(X_{2}+X_{1}\right)$. On the other hand, F must be large enough to avoid premature convergence. Several studies have concluded that using a value of 0.4 for the lower limit produces the highest success rate. ${ }^{[10]}$ Furthermore, this success rate continues to increase when $\mathrm{F}$ is continually assigned a random value between 0.4 and 1.0. ${ }^{[10]}$

By using Differential Evolution COMET increases the speed of convergence at a constant rate. COMET's Differential Evolution uses a strategy known as discrete recombination, also known as uniform crossover, to further modify the mutation vector population. There are two main strategies that can be selected to apply recombination: binomial and exponential. For either strategy, the probability of crossover, defined by the variable $\mathrm{Cr}$, can also be thought of as the mutation crossover rate and ranges from 0 to 1 . Binomial crossover, sometimes referred to as uniform crossover, randomly assigns a value between 0 and 1 to every parameter. If the value is less than or equal to $\mathrm{Cr}$, the new mutation vector takes that parameter from the current mutation vector, and if not, the parameter is taken from the original population member, see Figure 14(a). For exponential crossover, an optimization parameter in each member is chosen at random. All parameters prior to this are taken from the original population member and assigned to the new mutation vector. For the remaining parameters, a random value between 0 and 1 is assigned to each. Stepping through the parameters, while the random value is less 
than or equal to $\mathrm{Cr}$ the parameters are taken from the current mutation member. Once a value exceeds $\mathrm{Cr}$, all subsequent parameters are again taken from the original population member, see figure 14(b).

\begin{tabular}{|c|c|c|c|c|c|c|c|}
\hline rand & 0.81 & 0.91 & 0.17 & 0.92 & 0.63 & 0.09 & 0.28 \\
\hline Original & 42 & 16 & 39 & 113 & 20 & 6 & 73 \\
\hline & $\downarrow$ & $\downarrow$ & & $\downarrow$ & & & \\
\hline New & 42 & 16 & 9 & 113 & 45 & 12 & 90 \\
\hline Current & 17 & 28 & 9 & 85 & 45 & 12 & 90 \\
\hline
\end{tabular}

(a) Binomial crossover

\begin{tabular}{|c|c|c|c|c|c|c|c|}
\hline rand & & & 0.17 & 0.92 & 0.63 & 0.09 & 0.28 \\
\hline Original & 42 & 16 & 39 & 113 & 20 & 6 & 73 \\
\hline \multicolumn{8}{|c|}{$\downarrow$} \\
\hline New & 17 & 28 & 39 & 85 & 45 & 12 & 90 \\
\hline Current & 17 & 28 & 9 & 85 & 45 & 12 & 90 \\
\hline
\end{tabular}

(b) Exponential crossover

Figure 14: Examples of (a) binomial and (b) exponential crossover, with $\mathrm{Cr}=0.8$ and parameter 2 being randomly selected as the starting point

For either method, after the new mutation has been formed, each parameter must be checked to ensure that it still falls within the given search space. Any value found outside the search space is randomly assigned a new value between the upper and lower bound for that parameter.

The added complexity of the exponential crossover strategy allows the system to accommodate for functions that are decomposable. A decomposable function is comprised of optimization parameters that are highly independent of one another. For these functions, each parameter can be optimized independently and later combined to form the overall global optimum solution. In these situations, a low $\mathrm{Cr}$ value ranging 
from 0 to 0.2 , ensure a low mutation rate and produces the best results. For functions that are not decomposable, the overall cost of the function is dependent on the combination of parameters rather than the parameters individually. For these functions, the $\mathrm{Cr}$ value should range from 0.9 to 1 to emphasize mutation. Trajectory optimization problems in general are not decomposable, as each maneuver is highly dependent on the parameters before and after it. For example, a flyby is highly dependent on the date when the spacecraft left the previous planet and on the date when the spacecraft will reach the following planet. Deep space maneuvers, however, are decomposable. For a given set of date and time-of-flight parameters for a trajectory problem, such as the EVVEJDS, there exists a single set of values for $\alpha, \beta$, and $r_{p}$ that will optimize the entire trajectory. Programs like MGA use a $\mathrm{Cr}$ value of 0.5 by default to accommodate for both the decomposable and non-decomposable aspects of trajectories like these. COMET, on the other hand, allows the user to select an option to optimize the DSM parameters independent of the others. This allows the other $\mathrm{Cr}$ value to remain high in the range of 0.9 to 1.0 , while still achieving high quality results.

In addition to this selection option, COMET allows the user to choose from 10 possible Differential Evolution strategies. They consist of five methods that use either the exponential or binomial crossover strategies. The first two of these strategies, which have been previously mentioned, are $\mathrm{DE} / \mathrm{rand} / 1 / \mathrm{bin}$ and $\mathrm{DE} / \mathrm{rand} / 1 / \mathrm{exp}$. The rand part of this declaration notifies the program that the base member will be randomly selected. The 1 signifies that a single difference vector will be used to generate the mutation vector. The next options are $\mathrm{DE} / \mathrm{best} / 1 / \mathrm{bin}$ and $\mathrm{DE} / \mathrm{best} / 1 /$ exp. These are the same as the previous two except, the base member is always the best known solution. This option works well in 
single modal situations, but can have premature convergence for multi-modal functions. DE/rand/2/bin, DE/rand/2/exp, DE/best/2/bin, and DE/best/2/exp are similar to the methods listed above, however, each of these methods combine two differencing vectors during the mutation process. The final two methods are DE/rand-to-best/1/bin and DE/rand-to-best/1/exp. In these methods, the mutation vector is form by using each member in the population as the base member for its mutation vector. Like the DE/rand/1 methods these add a scaled difference vector from two randomly selected members. Additionally, the mutation scaling factor is multiplied by the difference between the base member and the best known member in the population. These two difference vectors are added to the base member to create the original mutation member population. To improve the computation efficiency of these methods, programs like MGA and COMET use population shuffling to generate the randomly selected vectors. This essentially uses a random permutation of the number of population members to reassign their positions in the population. The different methods can require anywhere from 2 to 5 shuffled populations based on the number of random population members each mutation requires.

Regardless of the strategy chosen, stopping criteria for the iteration process must be specified. Due to infeasibility to solve for the true global optimal solution for most interplanetary trajectory problems, the exact global optimum value is unknown. Therefore, this system has no way of telling if it has reached that solution. COMET allows the user to choose from three options. The optimization can stop after a specified number of iterations, a given amount of time, or a number of trials. A new trial is started when the optimization converges on a solution for at least 200 iterations. Since the convergence rates differ from problem to problem, each of the three stopping methods 
can have an effect on the accuracy of the final solution. Typically, iterations and time are closely correlated, but without knowing how long each iteration takes it may be hard to judge how long to specify prior to running the optimizer. Slower convergence rates require more time and more iterations to ensure the optimal solution is found. Therefore, unless the user is under a time constraint for producing the results, specifying the number of trials is generally the best option to choose.

As the iteration process progresses, a graph showing the cost of the best known trajectory for each iteration is continually updated in COMET's main function window. Additionally, the parameters and changes in velocity for each maneuver are displayed in a table next to the graph. Upon reaching the stopping criteria, the final solution is stored and passed to COMET's visualization function to create an animation of the trajectory and planetary orbits for the duration of the mission. 


\section{RESULTS}

The accuracy and robustness of COMET were evaluated by a multiple regression test and compared the results of five interplanetary trajectory design problems against the results previously produced by the MGA program from the European Space Agency's Advanced Concepts Team. ${ }^{[8]}$ The paper "Advanced Global Optimisation for Analysis and Design" goes into greater detail on the multiple global optimization methods used to produce ESA's results, as well as how their optimization strategies faired when computing the global minimum of several well known test functions. The CassiniHuygens mission served as the foundation for each of the six trajectory problems. The overall goal was to examine different sequences to complete a trajectory from Earth to Saturn while minimizing the total change in velocity, $\Delta \mathrm{V}$, required by the spacecraft. From this mission, the original periapse radius and eccentricity of the final parking orbit at Saturn, 108,950km and 0.98, respectfully, were used in each of the test cases. Each subsequent test case increased the complexity of the optimization by increasing the search space by an additional dimension. In the final test case, a DSM maneuver was added, resulting in an additional three dimensions in the search space. These cases included the following sequences: Earth-Jupiter-Saturn (EJS), Earth-Mars-Jupiter-Saturn (EMJS), Earth-Venus-Earth-Jupiter-Saturn (EVEJS), Earth-Venus-Venus-Earth-JupiterSaturn (EVVEJS), and Earth-Venus-DSM-Venus-Earth-Jupiter-Saturn (EVDVEJS). It is important to note that the procedures used in all test cases to evaluate the $\Delta \mathrm{V}$ 's for flybys differs between the two programs and consequently the same set of parameters will produce differing $\Delta \mathrm{V}$ 's. To account for this, the optimal solutions found by MGA were reevaluated using COMET's methodology so a direct comparison was made possible. 
The remainder of this section will examine the similarities and differences between these two programs.

\section{CASE I: Earth-Jupiter-Saturn (EJS)}

The first test case examined was an Earth-Jupiter-Saturn trajectory, which required the optimization of a search space with the following three dimensions: launch date (d0), time-of-flight between Earth and Jupiter (TOF1), and time-of-flight between Jupiter and Saturn (TOF2). The initial bounds for these dimensions can be found in Table 2. The search space for COMET was pruned by placing constraints on the launch $\Delta \mathrm{V}$ of $10 \mathrm{~km} / \mathrm{s}$ and $2 \mathrm{~km} / \mathrm{s}$ for all subsequent maneuvers, as well as constraining the altitude of the Jupiter flyby to no less than $10 \%$ of the planet's radius, and using a coarse grid sampling of 21 days for each of the three parameters. The new upper and lower bounds for the three dimensions are listed in Table 2. A visualization of the pruned search space can be seen in figures 15 and 16. The visualization of the arrival search space was omitted since it provided no additional information. With the search space pruned, COMET used a population of 100 members for the duration of 10000 iterations to optimize the parameters. The optimization population was reinitialized every time the program converged to within $0.1 \mathrm{~m} / \mathrm{s}$ for 100 consecutive iterations to avoid stagnation. This occurred 45 times with $100 \%$ of the converged solutions reaching the global optimum value of $9.3541 \mathrm{~km} / \mathrm{s}$. This shows significant improvement over the MGA program, which prematurely converged $50 \%$ of the time. See Figure 17 for the residual plot of best known solutions over the optimization process. It can be seen in Table 2, below, that the parameters of the converged solutions using MGA and COMET are roughly equivalent. Note that some of the upper limits for the pruned bounds exceed the 
initial upper limits. This results from unequal grid spacing when performing the search space pruning process.

Table 2: Bounds and best known solutions for EJS test case

\begin{tabular}{|l|r|r|r|r|r|r|}
\hline & \multicolumn{2}{|c|}{ Initial } & \multicolumn{2}{c|}{ Pruned } & \multicolumn{1}{l|}{ MGA } & \multicolumn{1}{l|}{ COMET } \\
\hline Parameters & Lower & Upper & Lower & Upper & Best & \multicolumn{1}{l|}{ Best } \\
\hline d0 [J2000] & -1278 & 547 & -984 & 276 & -177.308 & -177.29 \\
\hline TOF1 [days] & 99.7 & 1994.8 & 540 & 2010 & 911.8806 & 912.7411 \\
\hline TOF2 [days] & 366 & 7320.9 & 702 & 7338 & 4409.181 & 4445.976 \\
\hline Total DV (COMET) [km/s] & & & & & 9.40844 & 9.35405 \\
\hline Total DV (ESA) [km/s] & & & & & 9.35179 & \\
\hline
\end{tabular}

Valid Earth Launches

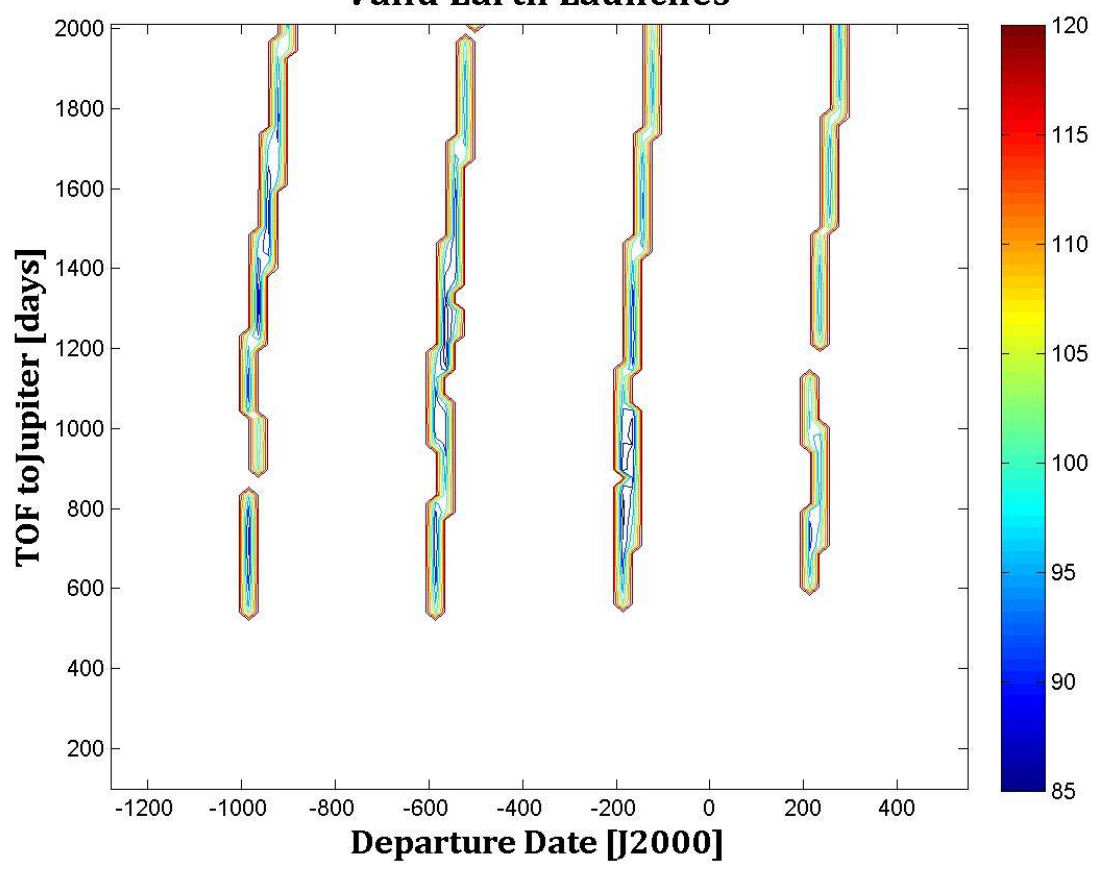

Figure 15: Valid Earth launch search space regions for EJS 


\section{Valid Jupiter Flybys}

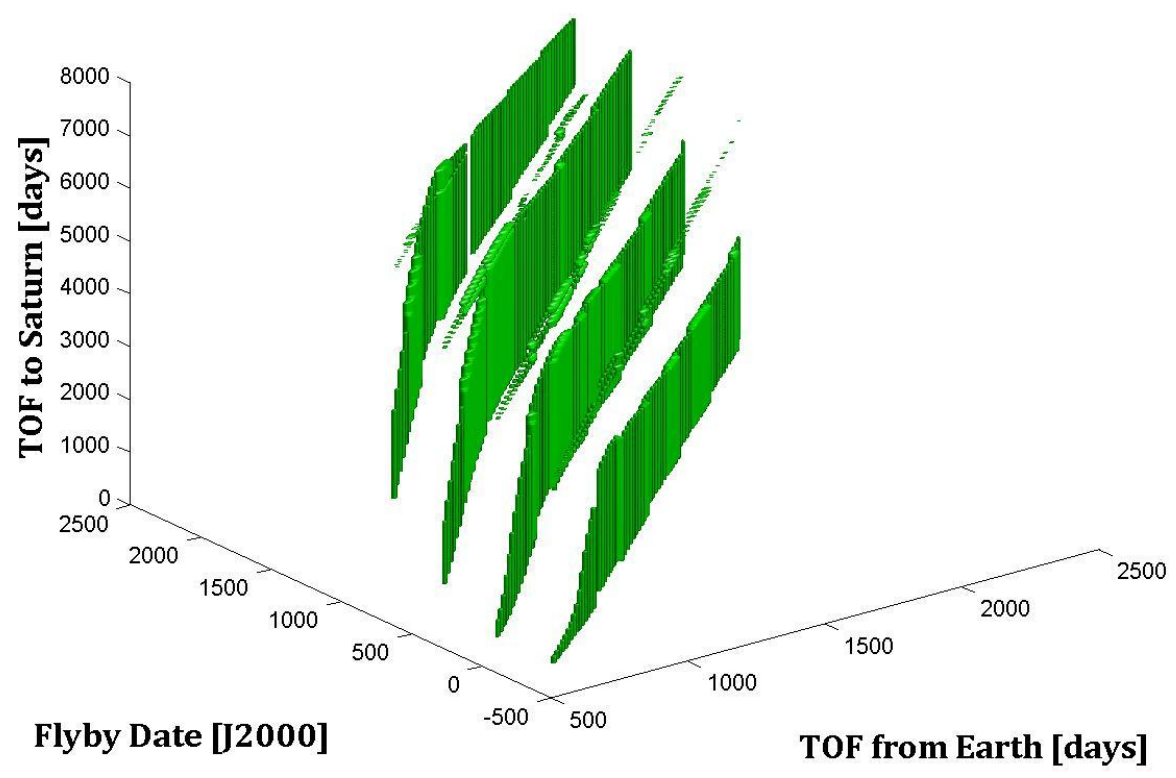

Figure 16: Valid Jupiter flyby search space regions for EJS

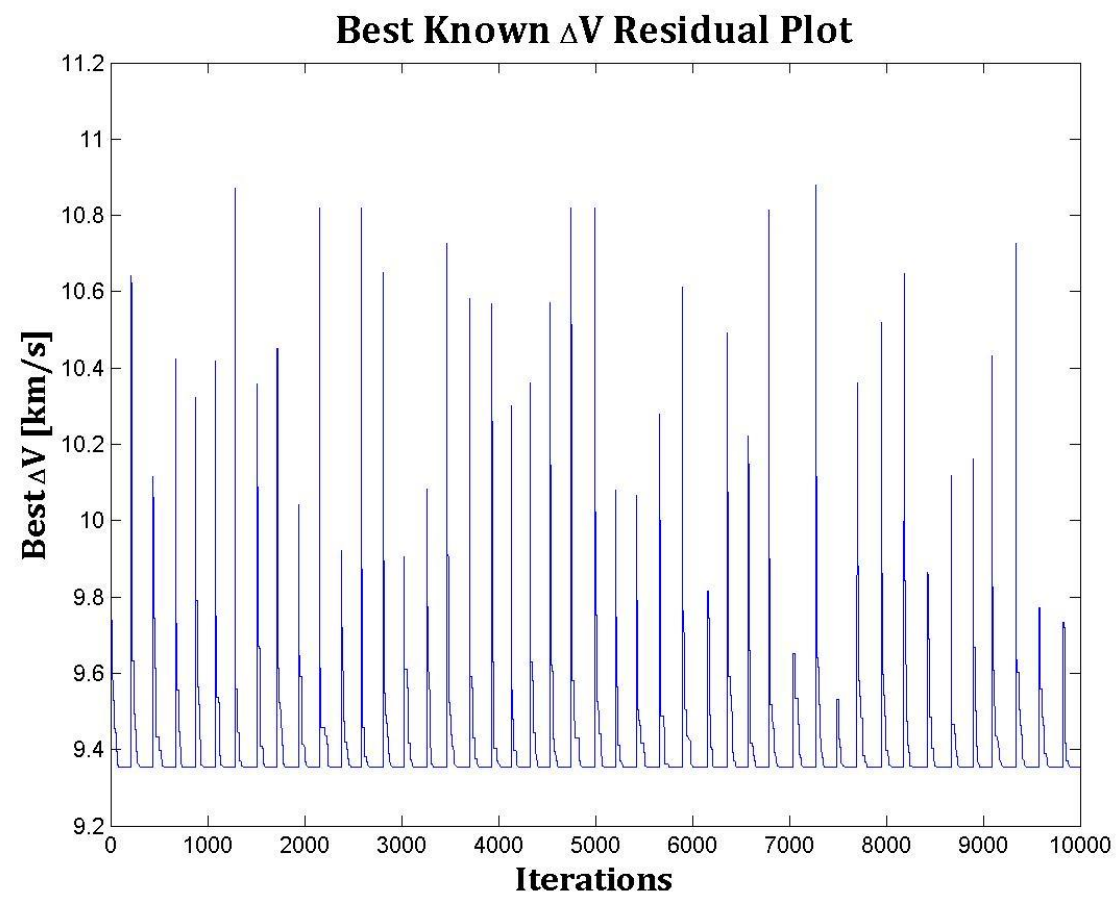

Figure 17: Residual plot of best known solutions for EJS 


\section{CASE II: Earth-Mars-Jupiter-Saturn (EMJS)}

An Earth-Mars-Jupiter-Saturn trajectory was the second case examined. This trajectory required the optimization of a search space with the following four dimensions: launch date (d0), time-of-flight between Earth and Mars (TOF1), time-of-flight between Mars and Jupiter (TOF2), and time-of-flight between Jupiter and Saturn (TOF3). The search space for COMET was pruned by using similar constraints as the EJS trajectory. The launch $\Delta \mathrm{V}$ was limited to $10 \mathrm{~km} / \mathrm{s}$ and all subsequent maneuvers were limited to 2 $\mathrm{km} / \mathrm{s}$. The altitudes of the Mars and Jupiter flybys were constrained to no less than $10 \%$ of the planets' radii, and again a coarse grid sampling of 21 days was used for each of the four parameters. The upper and lower bounds resulting from pruning the search space are listed in Table 3. The visualization of the pruned search space for EMJS can be seen in figures 18-20. Like the previous case a population of 100 members for the duration of 10000 iterations was used to optimize the parameters. Similarly, the optimization population was reinitialized every time the program converged to within $0.1 \mathrm{~m} / \mathrm{s}$ for 100 consecutive iterations. This occurred 7 fewer times compared to the EJS test case as a result of the increased complexity.

Furthermore, the probability of the system converging to the global optimal solution of $9.9465 \mathrm{~km} / \mathrm{s}$ was decreased to $97.4 \%$, with one of the trials prematurely converging. This still shows significant improvement over the MGA program, which prematurely converged more than $50 \%$ of the time. The residual plot of best known solutions over the optimization process can be seen in Figure 21. Table 3, below, displays the parameters of the converged solutions using MGA and COMET. Again the two programs converged to within $1.1 \%$ of the same solution. The individual break down of 
$\Delta \mathrm{V}$ 's shows that the majority of the discrepancy between $\Delta \mathrm{V}$ 's occurs at the Mars flyby. This results from the differing techniques of the two programs to deal with the fact that the altitude necessary to perform this flyby would cause the spacecraft to collide with the planet. To prevent this from happening COMET calculates the change in velocity necessary to maintain the desired course using a minimum approach flyby. MGA on the other hand determines the altitude from a gradient based search using both the inbound and outbound velocities. It applies a compensation $\Delta \mathrm{V}$ factor for flybys that drop below the minimum allowed altitude. Later versions of COMET will attempt to combine the two methods to produce the most accurate and lowest possible cost associated with flybys like these.

Table 3: Bounds and best known solutions for EMJS test case

\begin{tabular}{|l|c|c|c|c|l|l|}
\hline & \multicolumn{2}{|c|}{ Initial } & \multicolumn{2}{c|}{ Pruned } & MGA & COMET \\
\hline Parameters & Lower & Upper & Lower & Upper & Best & Best \\
\hline d0 [J2000] & -1278 & 547 & -1278 & 444 & -1249.87 & -1247.59 \\
\hline TOF1 [days] & 25.9 & 1035.5 & 970 & 1054 & 1000.963 & 1004.078 \\
\hline TOF2 [days] & 112.6 & 2252.7 & 679 & 2233 & 1116.975 & 1092.907 \\
\hline TOF3 [days] & 336 & 7320.9 & 1386 & 7329 & 4974.14 & 5025.034 \\
\hline Total DV (COMET) $[\mathrm{km} / \mathrm{s}]$ & & & & & 10.05749 & 9.946551 \\
\hline Total DV (ESA) $[\mathrm{km} / \mathrm{s}]$ & & & & & 9.185971 & \\
\hline
\end{tabular}




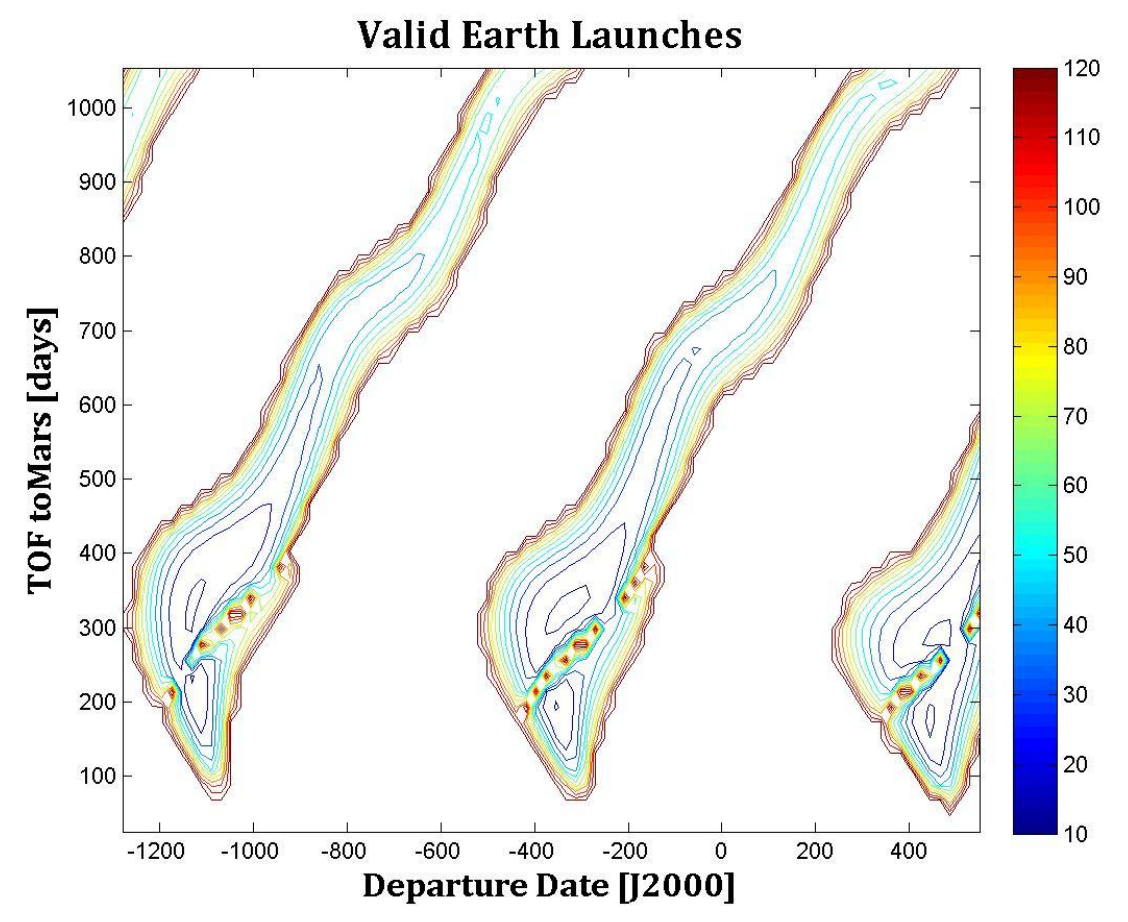

Figure 18: Valid Earth launch search space regions for EMJS

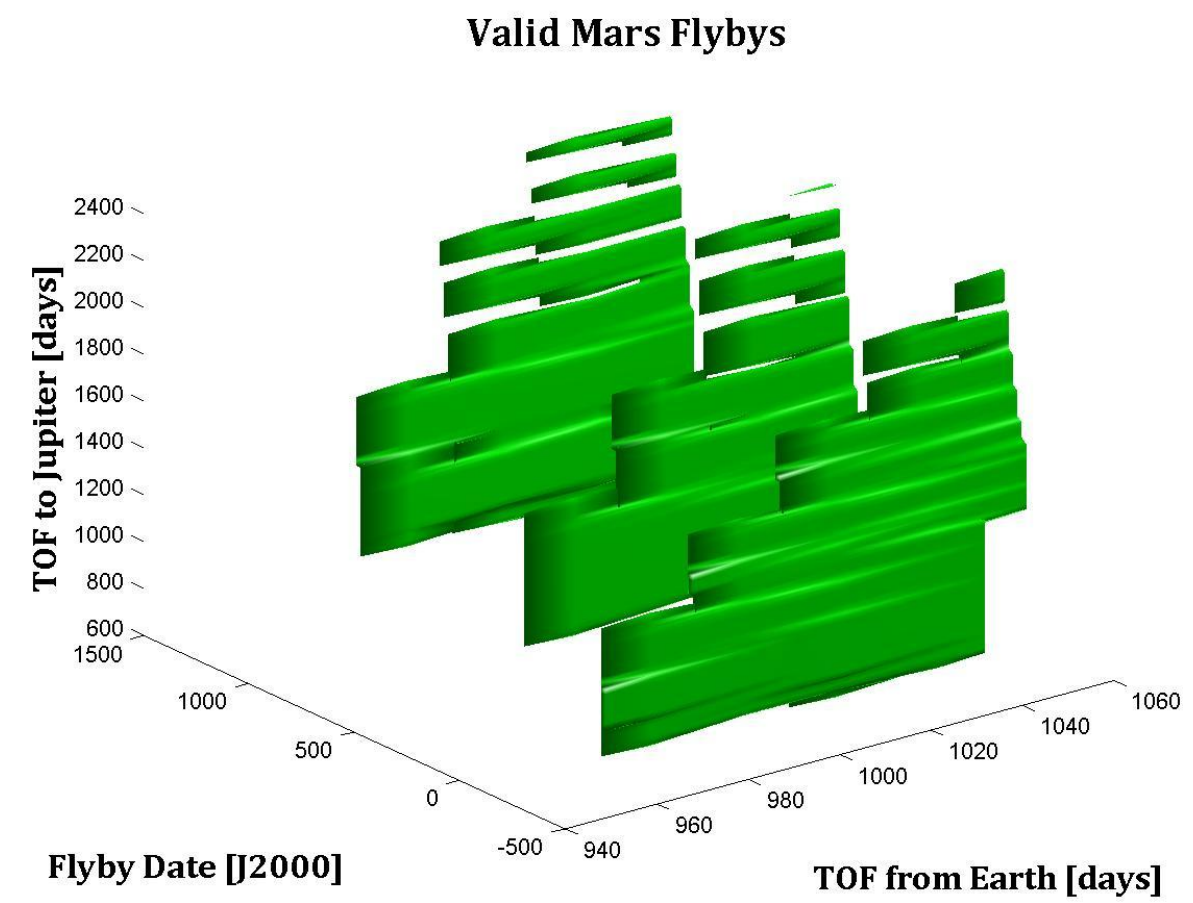

Figure 19: Valid Mars flyby search space regions for EMJS 


\section{Valid Jupiter Flybys}

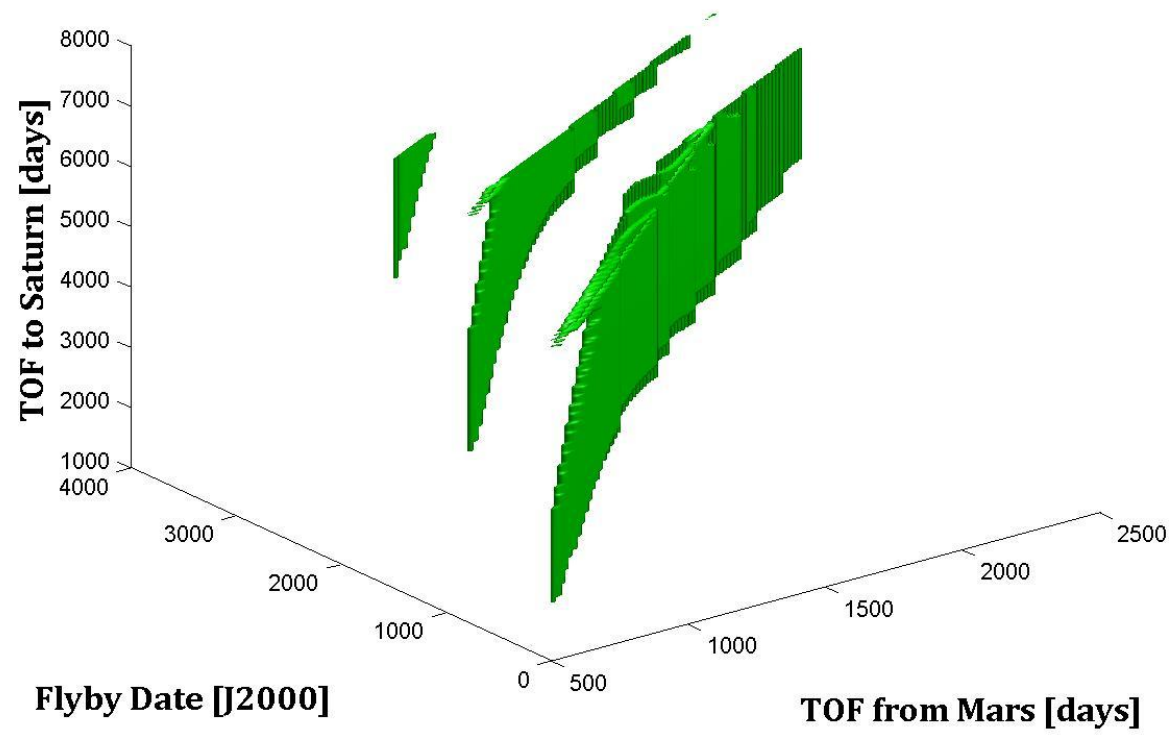

Figure 20: Valid Jupiter flyby search space regions for EMJS

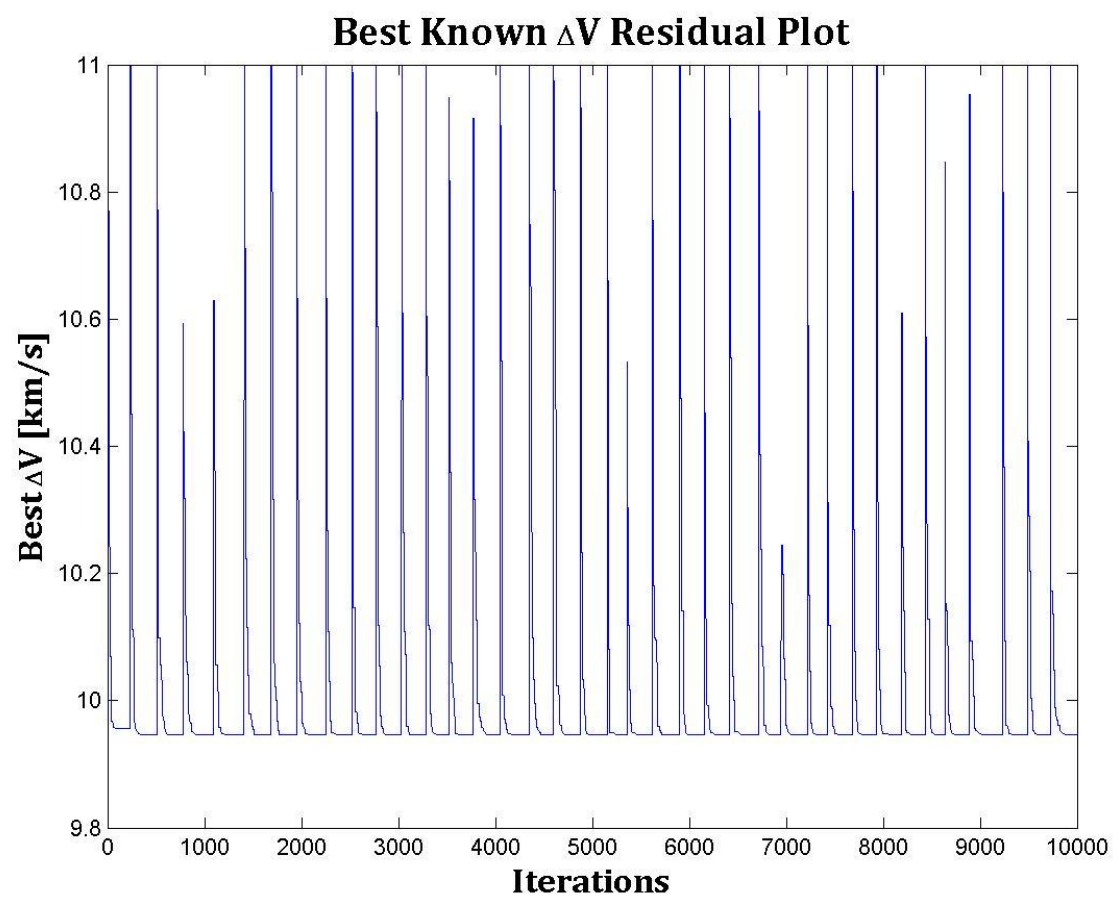

Figure 21: Residual plot of best known solutions for EMJS 


\section{CASE III: Earth-Venus-Earth-Jupiter-Saturn (EVEJS)}

The third case tested was an Earth-Venus-Earth-Jupiter-Saturn trajectory. This trajectory required the optimization of a search space with the following five dimensions: launch date (d0), time-of-flight between Earth and Venus (TOF1), time-of-flight between Venus and Earth (TOF2), time-of-flight between Earth and Jupiter (TOF3), and time-offlight between Jupiter and Saturn (TOF4). The search space for COMET was pruned by using similar constraints as the previous two cases. The launch $\Delta \mathrm{V}$ was limited to 10 $\mathrm{km} / \mathrm{s}$ and all subsequent maneuvers were limited to $2 \mathrm{~km} / \mathrm{s}$. The altitudes of the Venus, Earth, and Jupiter flybys were constrained to no less than $10 \%$ of the planets' radii, and again a coarse grid sampling of 21 days was used for each of the five parameters. The upper and lower bounds resulting from pruning the search space are listed in Table 4. The visualization of the pruned search space for EMJS can be seen in figures 22-25. As with the two previous cases, a population of 100 members for the duration of 10000 iterations was used to optimize the parameters. Similarly the optimization population was reinitialized every time the program converged to within $0.1 \mathrm{~m} / \mathrm{s}$ for 100 consecutive iterations. This occurred 26 times with the convergence to the global optimum value of $6.1832 \mathrm{~km} / \mathrm{s}$ only occurring $11.5 \%$ of the time. This extremely low probability results from a highly isolated solution. This is strong example of how COMET's pruning of the search space increases the quality of the optimization process. Even though the probability is low, COMET still managed to locate a solution that MGA overlooked in every one of its trials. The residual plot of best known solutions over the optimization process can be seen in figure 26. Table 4, below, displays the parameters of the converged solutions using MGA and COMET. It can be seen that the first three 
parameters are roughly the same for the two programs, however, the significant change in total $\Delta \mathrm{V}$ results from the difference in the TOF3 and TOF4 parameters.

Table 4: Bounds and best known solutions for EVEJS test case

\begin{tabular}{|l|c|c|c|c|l|l|}
\hline & \multicolumn{2}{|c|}{ Initial } & \multicolumn{2}{c|}{ Pruned } & MGA & COMET \\
\hline Parameters & Lower & Upper & Lower & Upper & Best & Best \\
\hline d0 [J2000] & -1278 & 547 & -1131 & 150 & -229.498 & -229.374 \\
\hline TOF1 [days] & 14.6 & 584.3 & 140 & 329 & 158.0007 & 157.858 \\
\hline TOF2 [days] & 14.6 & 584.3 & 56 & 602 & 288.9699 & 286.3128 \\
\hline TOF3 [days] & 99.7 & 1994.9 & 393 & 1863 & 1671.395 & 1267.829 \\
\hline TOF4 [days] & 366 & 7320.9 & 681 & 7338 & 6424.877 & 6279.491 \\
\hline Total DV (COMET) $[\mathrm{km} / \mathrm{s}]$ & & & & & 9.772332 & 6.183214 \\
\hline Total DV (ESA) $[\mathrm{km} / \mathrm{s}]$ & & & & & 7.548365 & \\
\hline
\end{tabular}

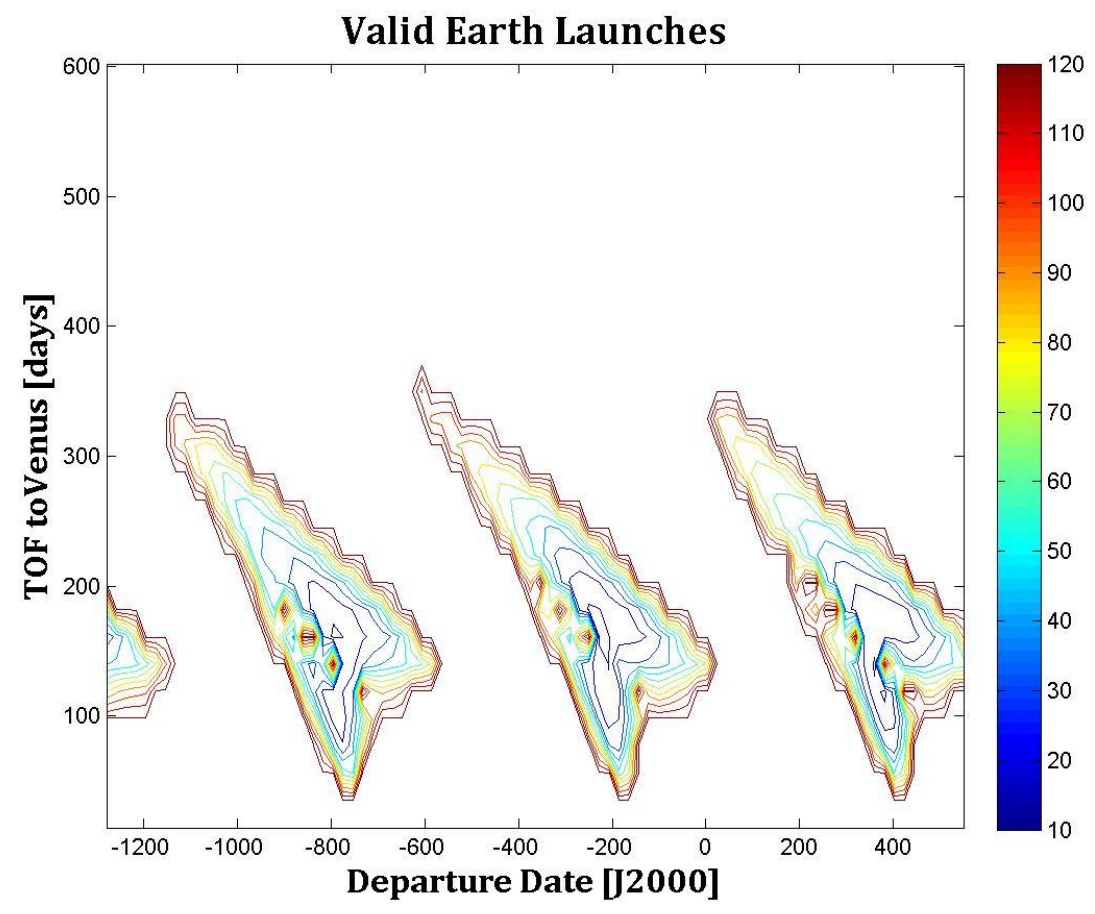

Figure 22: Valid Earth launch search space regions for EVEJS 


\section{Valid Venus Flybys}

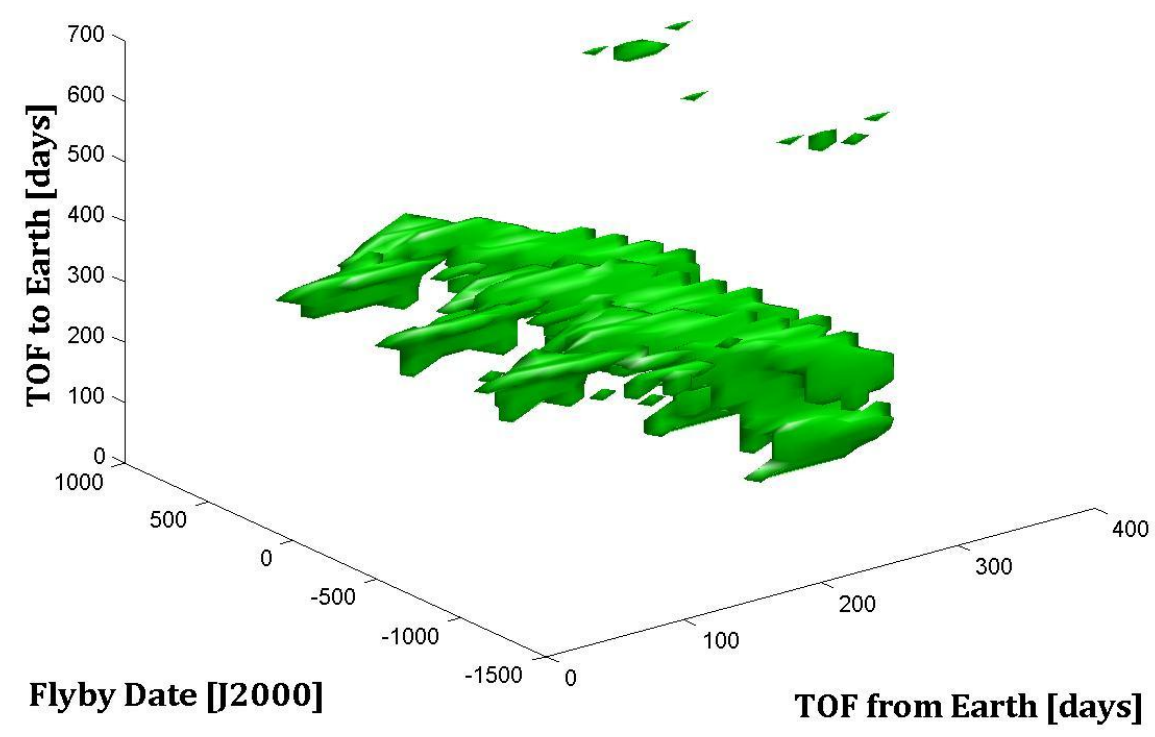

Figure 23: Valid Venus flyby search space regions for EVEJS

\section{Valid Earth Flybys}

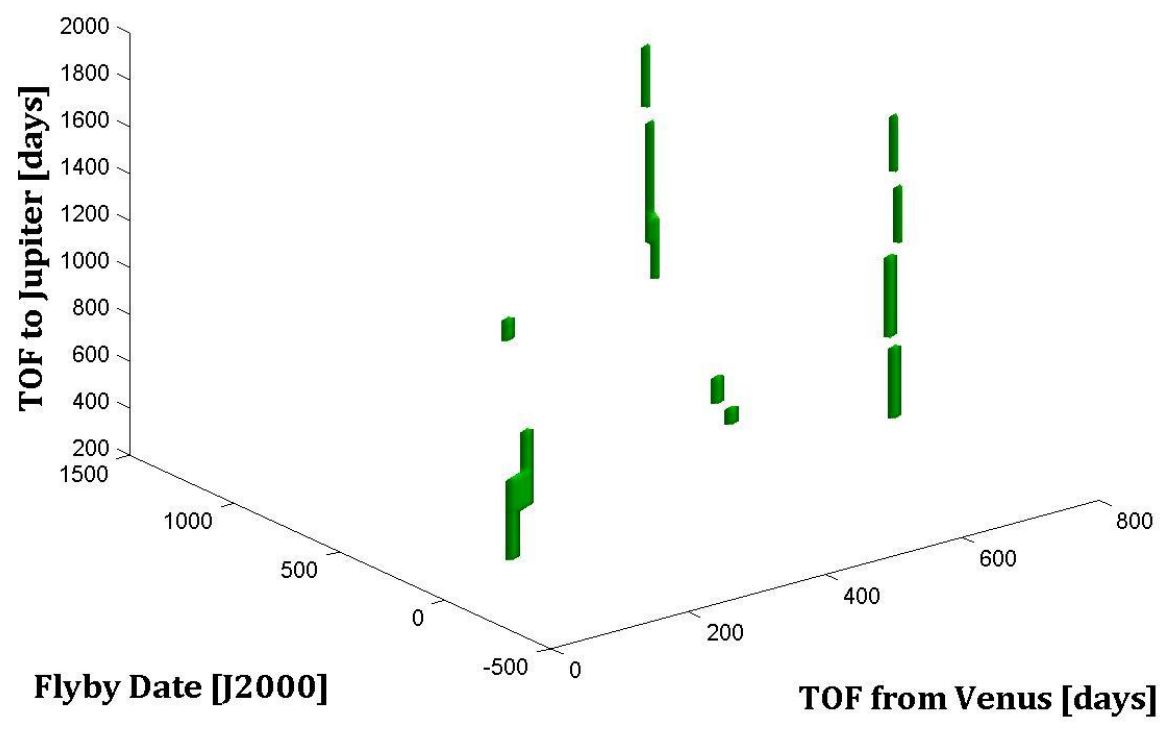

Figure 24: Valid Earth flyby search space regions for EVEJS 


\section{Valid Jupiter Flybys}

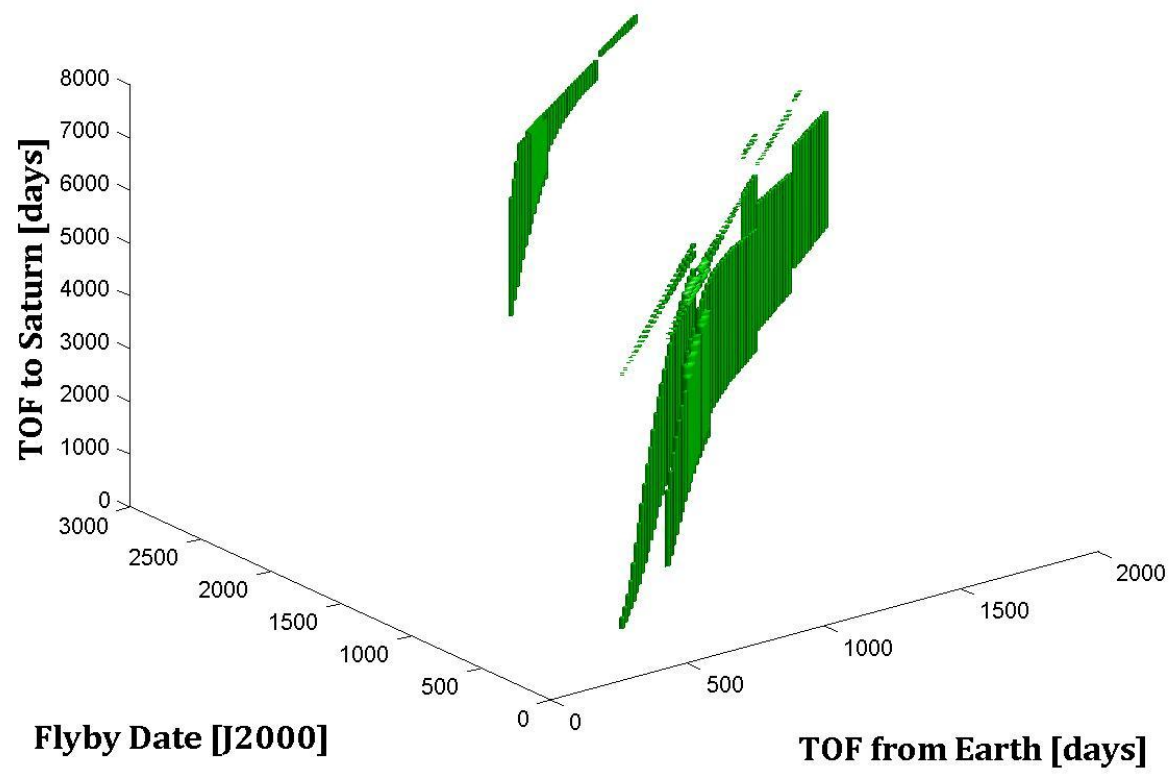

Figure 25: Valid Jupiter flyby search space regions for EVEJS

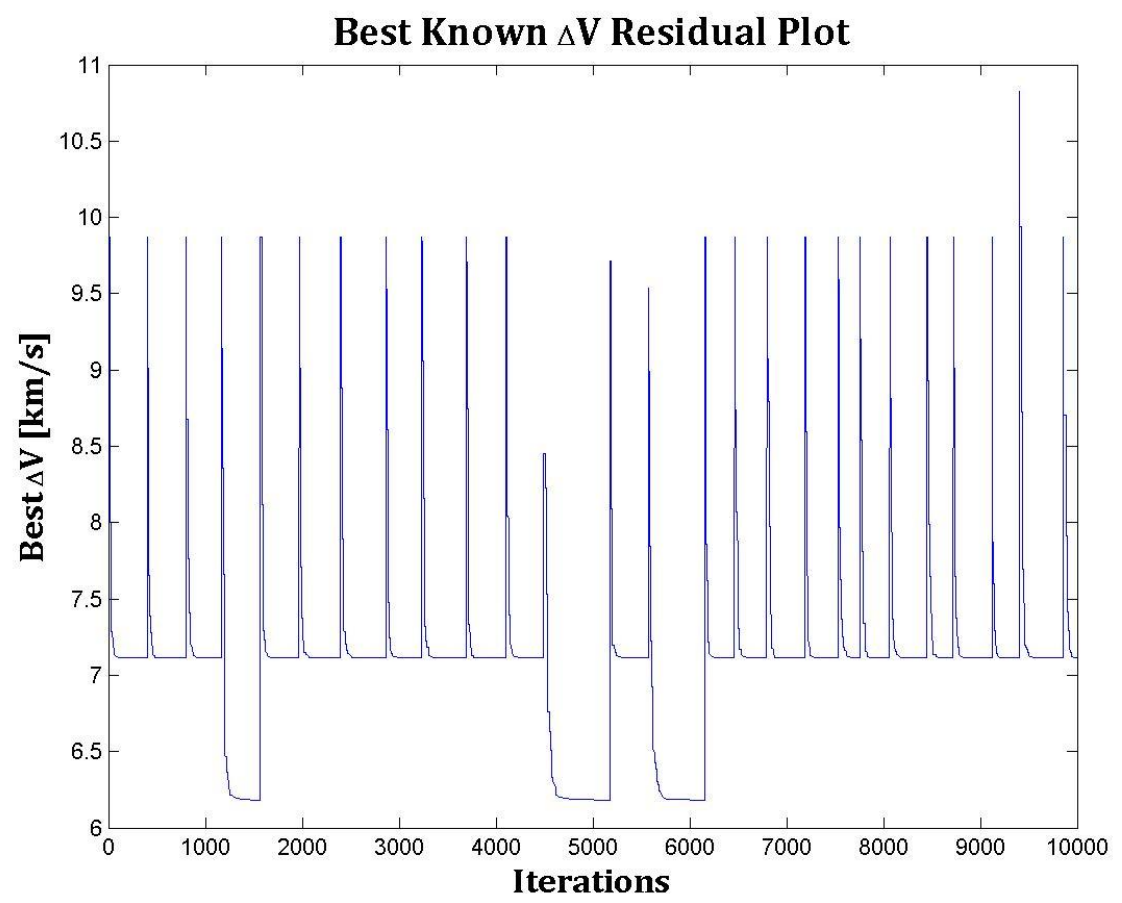

Figure 26: Residual plot of best known solutions for EVEJS 


\section{CASE IV: Earth-Venus-Venus-Earth-Jupiter-Saturn (EVVEJS)}

The next test case was an Earth-Venus-Venus-Earth-Jupiter-Saturn trajectory. This trajectory required the optimization of a search space with the following six dimensions: launch date (d0), time-of-flight between Earth and Venus (TOF1), time-offlight between Venus and Venus (TOF2), time-of-flight between Venus and Earth (TOF3), time-of-flight between Earth and Jupiter (TOF4), and time-of-flight between Jupiter and Saturn (TOF5). The search space for COMET was pruned by using similar constraints as the previous cases. The launch $\Delta \mathrm{V}$ was limited to $10 \mathrm{~km} / \mathrm{s}$ and all subsequent maneuvers were limited to $2 \mathrm{~km} / \mathrm{s}$. The altitudes of the Venus, Earth, and Jupiter flybys were constrained to no less than $10 \%$ of the planets' radii, and again a coarse grid sampling of 21 days was used for each of the five parameters. The upper and lower bounds resulting from pruning the search space are listed in Table 5. The visualization of the pruned search space for EMJS can be seen in figures 27-31. As with two previous cases a population of 100 members for the duration of 10000 iterations was used to optimize the parameters. Likewise, the optimization population was reinitialized every time the program converged to within $0.1 \mathrm{~m} / \mathrm{s}$ for 100 consecutive iterations. The increased complexity of this problem resulted in the reinitialization of the population occurring only 20 times with the convergence to the global optimum value of 5.8858 $\mathrm{km} / \mathrm{s}$ only occurring $10 \%$ of the time. Similar to the EVEJS case, this extremely low probability results from a highly isolated solution, yet due to the increased dimensionality of this problem the assumed premature convergence was a result of a slow convergence rate. The residual plot of best known solutions over the optimization process can be seen in Figure 32. From this figure it is easy to see the much slower convergence time 
compared to that of the earlier cases. Table 4 below displays the parameters of the converged solutions using MGA and COMET. With the exception of TOF5, the other parameters are in roughly the same areas of the search space with values differing only on the order of a couple weeks.

Table 5: Bounds and best known solutions for EVVEJS test case

\begin{tabular}{|l|r|r|r|r|r|l|}
\hline & \multicolumn{2}{|c|}{ Initial } & \multicolumn{2}{c|}{ Pruned } & \multicolumn{1}{l|}{ MGA } & \multicolumn{1}{l|}{ COMET } \\
\hline Parameters & Lower & Upper & Lower & Upper & Best & \multicolumn{1}{l|}{ Best } \\
\hline d0 [J2000] & -1278 & 547 & -1110 & 255 & -802.633 & -778.824 \\
\hline TOF1 [days] & 14.6 & 584.3 & 98 & 350 & 199.4685 & 180.7817 \\
\hline TOF2 [days] & 22.5 & 898.7 & 400 & 673 & 419.9938 & 413.8924 \\
\hline TOF3 [days] & 14.6 & 584.3 & 56 & 602 & 56.1717 & 56.00003 \\
\hline TOF4 [days] & 99.7 & 1994.9 & 393 & 2010 & 976.9088 & 977.8947 \\
\hline TOF5 [days] & 366 & 7320.9 & 681 & 7338 & 4369.162 & 4496.324 \\
\hline Total DV (COMET) $[\mathrm{km} / \mathrm{s}]$ & & & & & 6.688009 & 5.855822 \\
\hline Total DV (ESA) $[\mathrm{km} / \mathrm{s}]$ & & & & & 6.141987 & \\
\hline
\end{tabular}

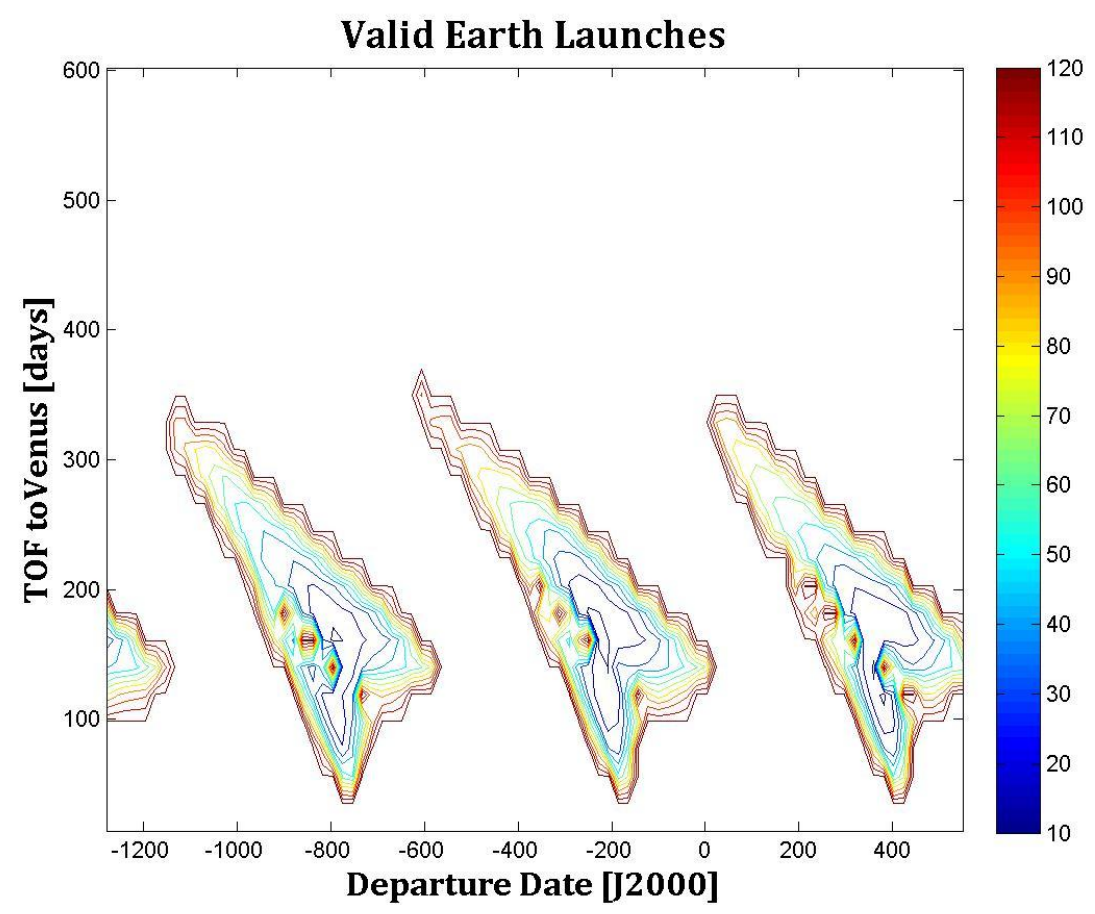

Figure 27: Valid Earth launch search space regions for EVVEJS 


\section{Valid Venus Flybys}

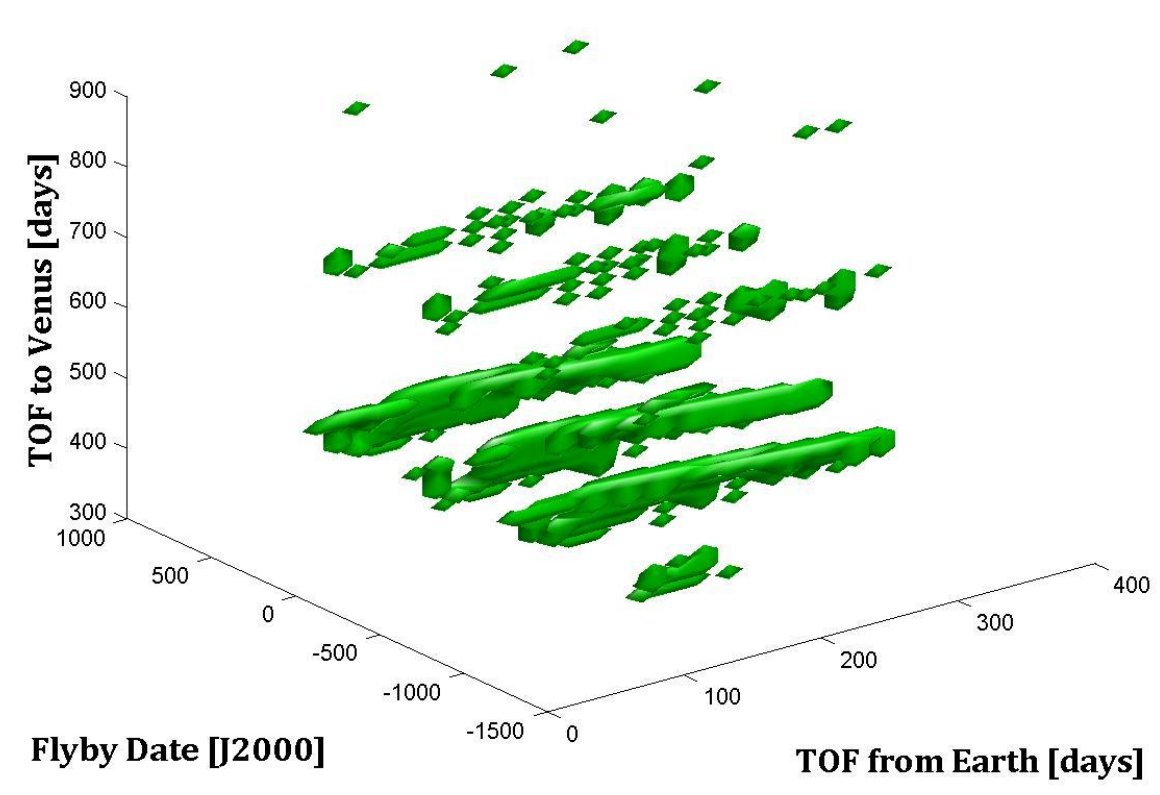

Figure 28: Valid Venus flyby search space regions for EVVEJS

\section{Valid Venus Flybys}

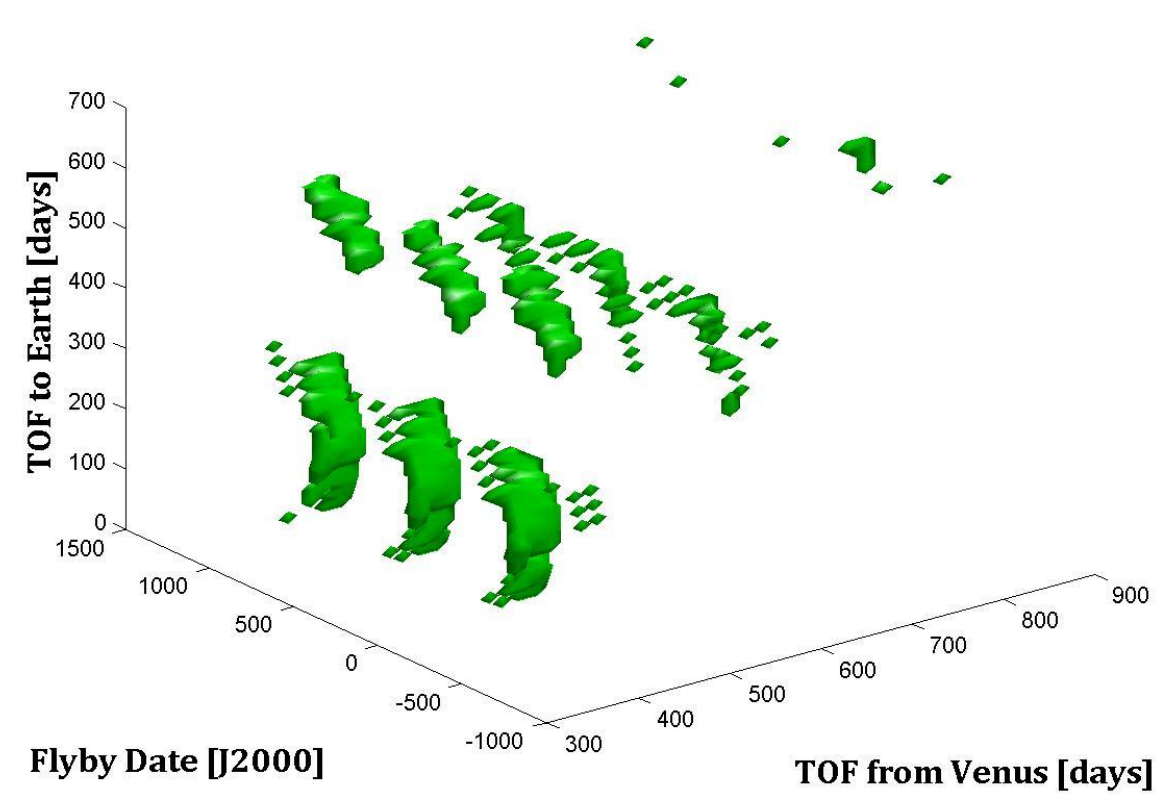

Figure 29: Valid Venus flyby search space regions for EVVEJS 


\section{Valid Earth Flybys}

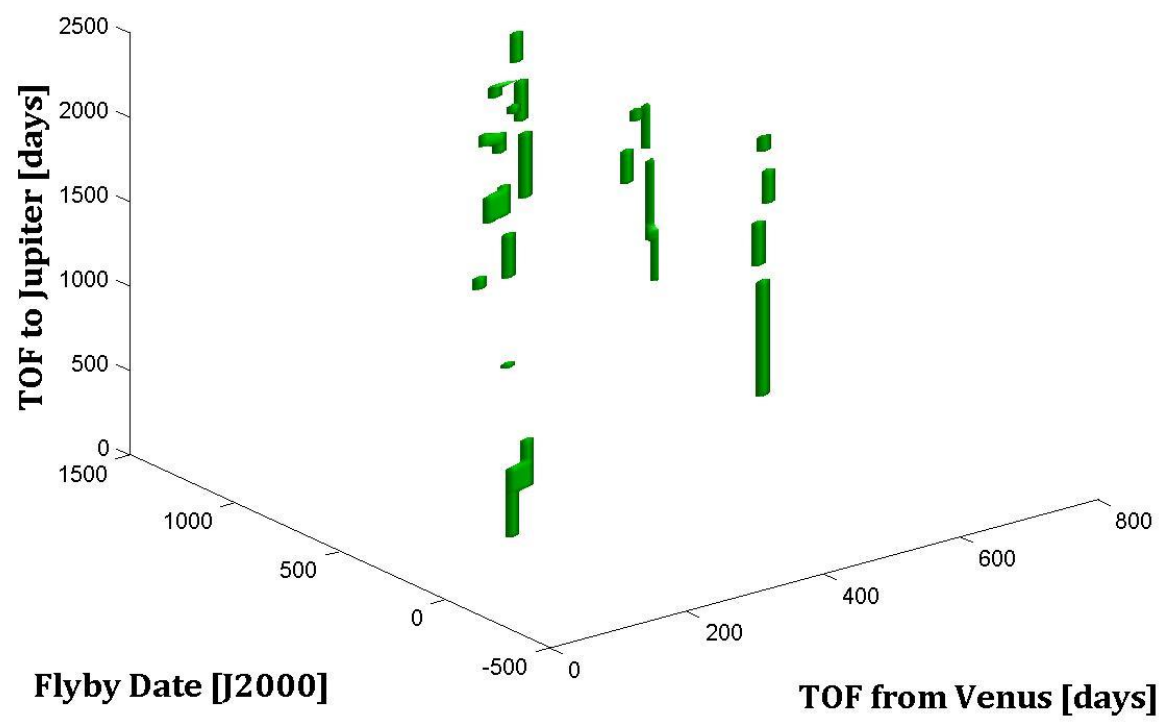

Figure 30: Valid Earth flyby search space regions for EVVEJS

\section{Valid Jupiter Flybys}

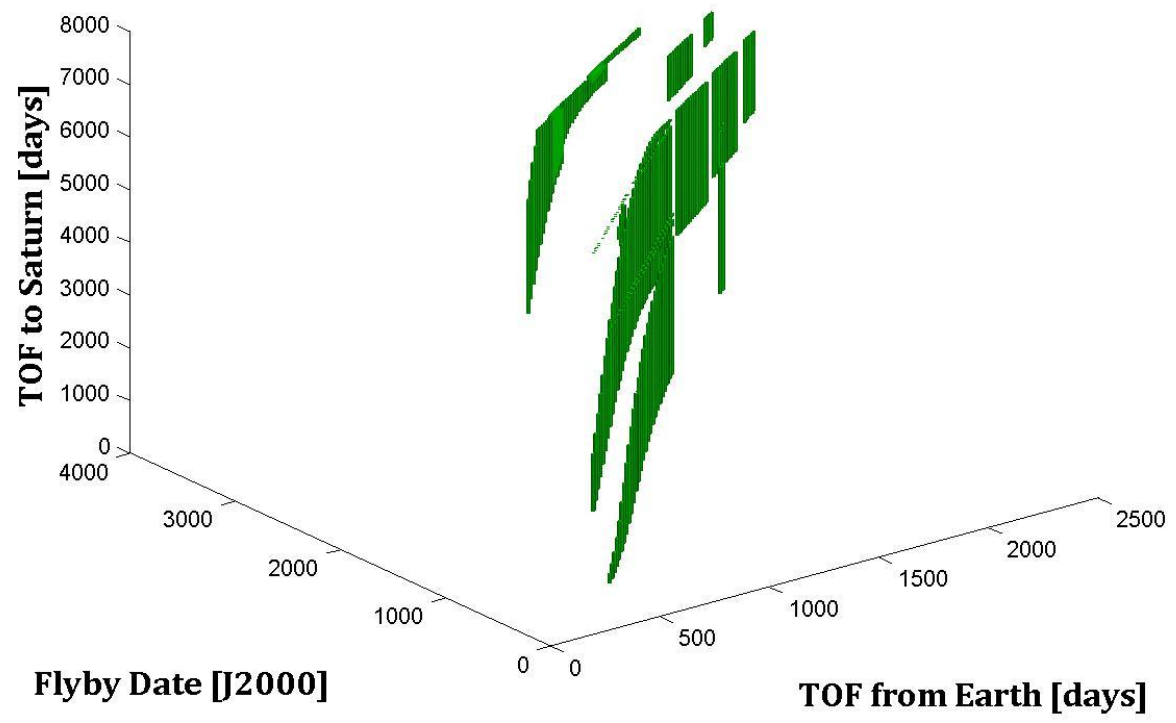

Figure 31: Valid Jupiter flyby search space regions for EVVEJS 


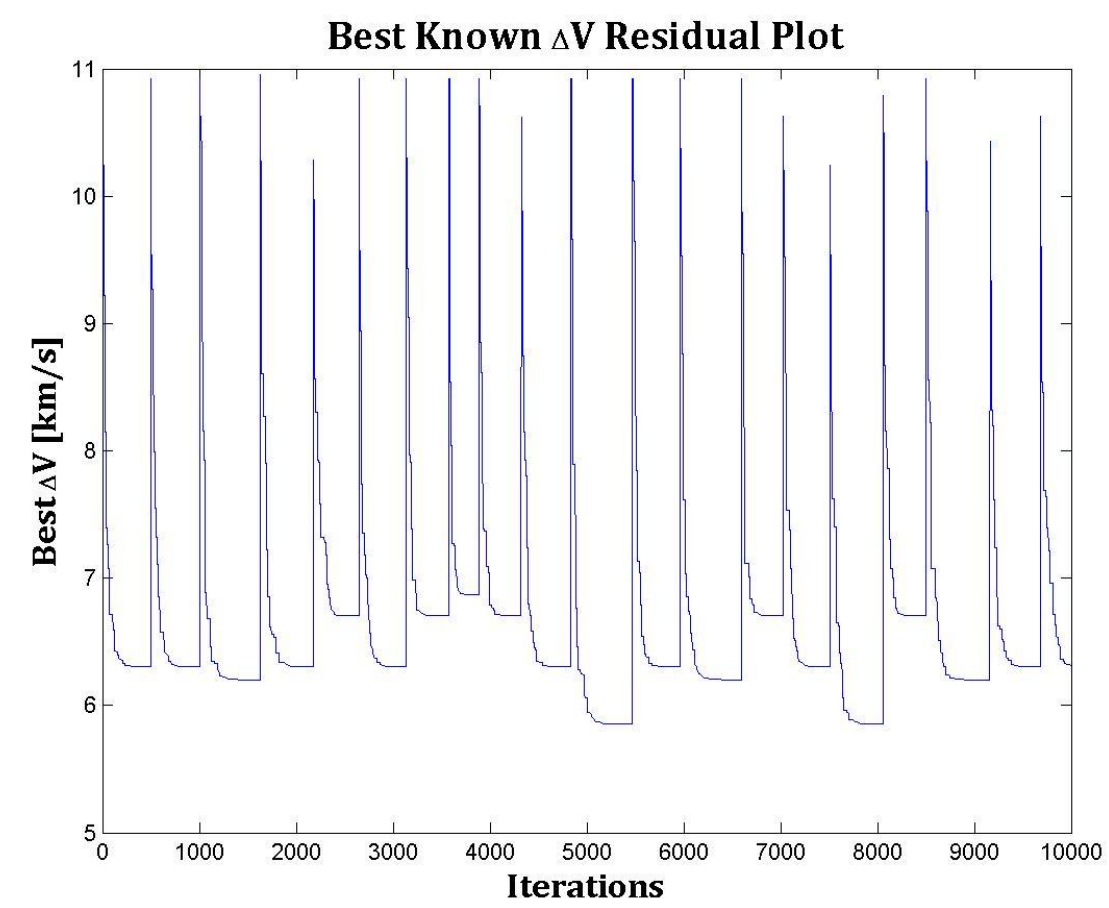

Figure 32: Residual plot of best known solutions for EVVEJS

\section{CASE V: Earth-Venus-DSM-Venus-Earth-Jupiter-Saturn (EVDVEJS)}

The final and most complex test case was an Earth-Venus-DSM-Venus-EarthJupiter-Saturn trajectory. This nine-dimensional test case used the same initial bounds and conditions as the EVVEJS case for launch dates and times-of-flight, while adding $\alpha$, $\beta$, and $1 / \mathrm{rp}$ as parameters to the optimization. COMET ran the test twice, allowing the DSM parameters to be decomposed from the trajectory problem and optimized independently. However, due to the computationally expensive nature of optimizing each set of DSM parameters for each population member in every iterations, COMET used a tiered process to determine when and which members to optimize. This process included the optimization of the parameters for the best member in each iteration, all members every 10 iterations, a higher level optimization of the best member every 10 iterations, 
and an even higher level of optimization when the system was about to converge. While the decomposition of the DSM caused the program to take approximately twice the run time compared to optimizing without decomposing, the resulting differences were significant. The residual plots for both tests can be seen in figures 33 and 34 . Even though the best found solution for the decomposed test was only $20 \mathrm{~m} / \mathrm{s}$ less than the $4.792 \mathrm{~km} / \mathrm{s}$ result from the non-decomposed test, from the residual plots it is clear to see that by decomposing the DSM, the average value of the best member was reduced by more than $1200 \mathrm{~m} / \mathrm{s}$. Additionally, it can be noted that the decomposed test converged to solutions less than $6 \mathrm{~km} / \mathrm{s} 100 \%$ of the time, whereas when the DSM parameters were not decomposed only $40 \%$ of the converged solutions were less than $6 \mathrm{~km} / \mathrm{s}$.

Table 6: Bounds and best known solutions for EVDVEJS test case

\begin{tabular}{|c|c|c|c|c|c|c|c|}
\hline & \multicolumn{2}{|c|}{ Initial } & \multicolumn{2}{|c|}{ Pruned } & \multirow{2}{*}{$\begin{array}{l}\text { MGA } \\
\text { Best }\end{array}$} & \multicolumn{2}{|l|}{ COMET } \\
\hline Parameters & Lower & Upper & Lower & Upper & & No Decomp & Decomp \\
\hline $\mathrm{d} 0$ [J2000] & -1278 & 547 & -1110 & 255 & -796.464 & -795.17 & -805.719 \\
\hline TOF1 [days] & 14.6 & 584.3 & 98 & 350 & 188.1997 & 150.6932 & 192.8607 \\
\hline TOF2 [days] & 22.5 & 898.7 & 400 & 673 & 428.536 & 459.5583 & 428.4731 \\
\hline TOF3 [days] & 14.6 & 584.3 & 56 & 602 & 52.9901 & 56.26422 & 56.03189 \\
\hline TOF4 [days] & 99.7 & 1994.9 & 393 & 2010 & 1004.438 & 956.0365 & 970.6841 \\
\hline TOF5 [days] & 366 & 7320.9 & 681 & 7338 & 4508.694 & 4443.314 & 4481.038 \\
\hline$\alpha$ & 0.1 & 0.9 & 0.1 & 0.9 & 0.49117 & 0.321537 & 0.508065 \\
\hline $1 / \mathrm{rp}$ & $1 / 100$ & $1 / 1.1$ & $1 / 100$ & $1 / 1.1$ & 0.858117 & 0.800393 & 0.750089 \\
\hline$\beta$ & $-\pi$ & $\pi$ & $-\pi$ & $\pi$ & -0.79219 & -0.21885 & 0.713497 \\
\hline $\begin{array}{l}\text { Total DV } \\
\text { (COMET) }[\mathrm{km} / \mathrm{s}]\end{array}$ & & & & & 4.589063 & 4.792723 & 4.752619 \\
\hline $\begin{array}{l}\text { Total DV } \\
(\text { ESA) }[\mathrm{km} / \mathrm{s}]\end{array}$ & & & & & 5.032349 & & \\
\hline
\end{tabular}




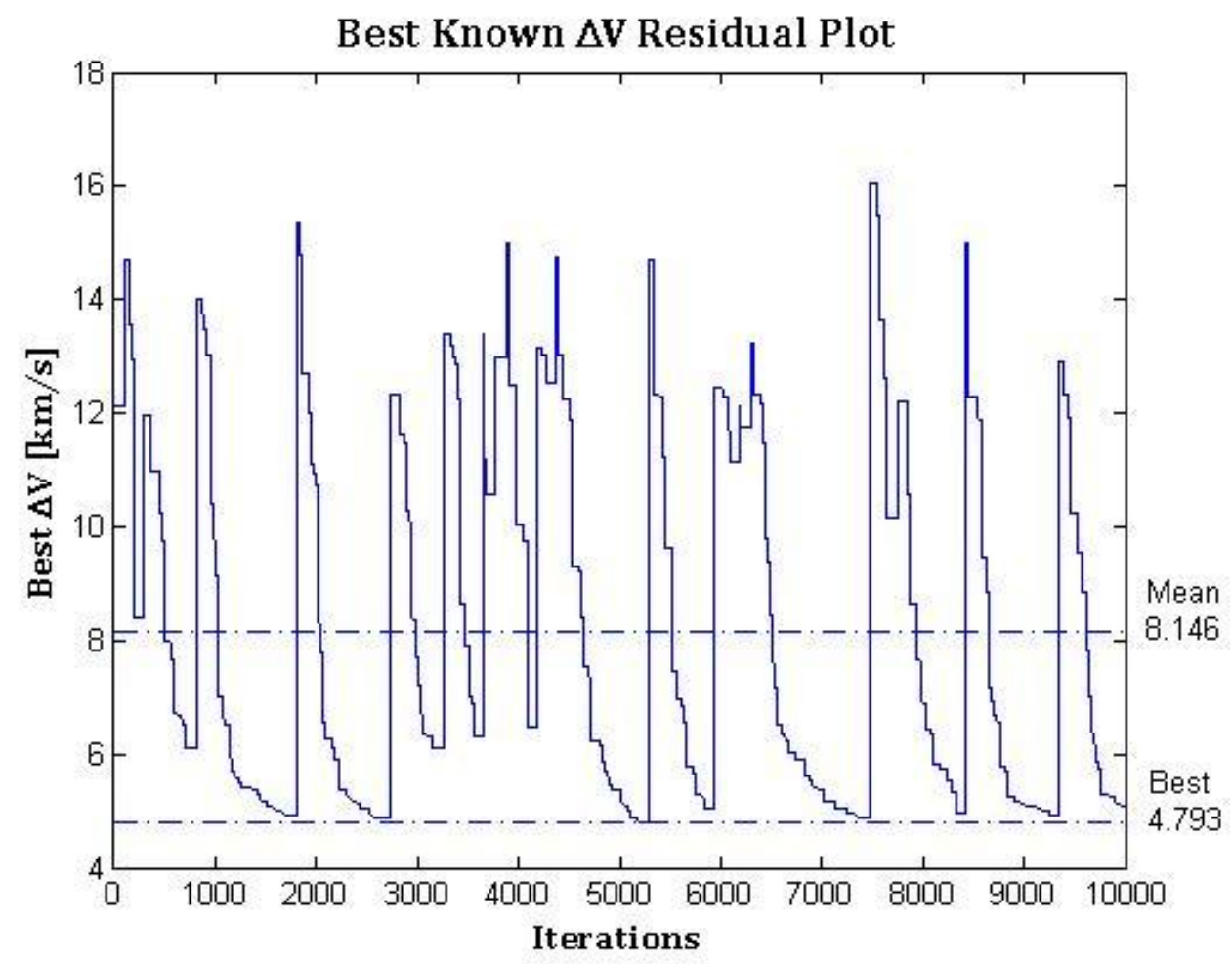

Figure 33: Residual plot of best known solutions for EVDVEJS without decomposing

Best Known $\Delta V$ Residual Plot

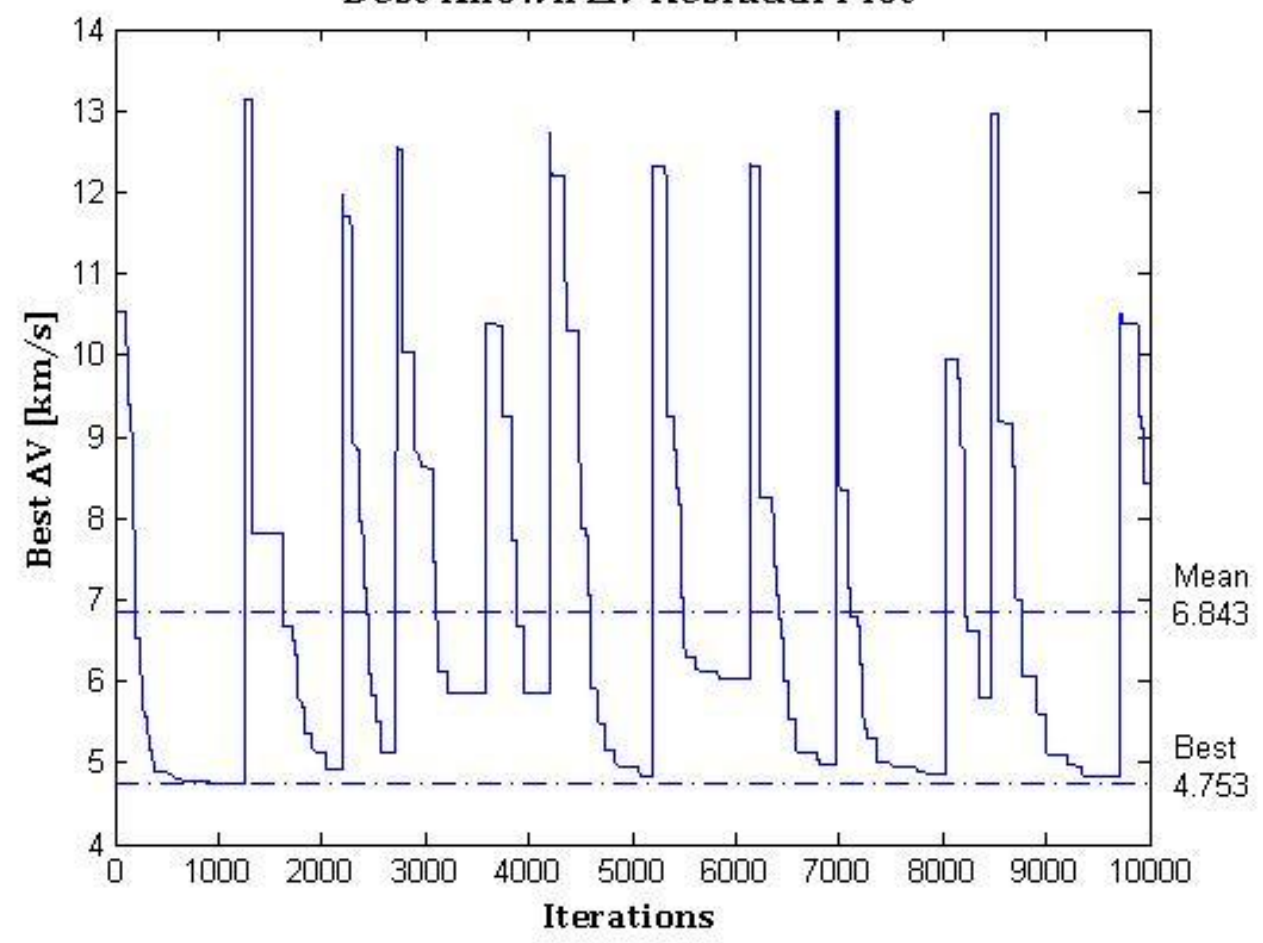

Figure 34: Residual plot of best known solutions for EVDVEJS with decomposing 


\section{Multiple Linear Regression Analysis:}

A test was constructed to evaluate the effectiveness that population size, continuously randomizing the mutation scaling factor, high crossover probability, and pruning the search space had on the quality of COMET's Differential Evolution global optimizer. The test consisted of 200 trials replicating eight designs 25 times each. For each trial, a population was assigned a random population size ranging from 100 to 1000 members. Additionally, each trial was assigned three binary indicators: Random_F, High_Cr, and Pruned. A value of 0 for Random_F specified that the mutation scaling factor would be held at a constant value of 0.8 , whereas a value of 1 indicated that the mutation scaling factor would be continuously randomized throughout the trial. A value of 0 or 1 for High_Cr identified that the current trial would use a crossover probability of 0.5 or 0.9 , respectfully. Finally, a value of 0 for Pruned indicated that the initial population and bounds would be taken from the original values specified in the set up of the EVVEJS problem. A value of 1 for Pruned, on the other hand, signified that the initial population and bounds would utilize the pruned search space information. The eight unique possibilities of the three indicators, shown in Table 7, established the aforementioned replicated designs.

Each trial was allowed to optimize for 100 iterations. This value was selected such that it would be long enough to allow the populations to begin to mature, while remaining short enough to reduce the chance of having the populations converge to the optimal solution. Once all 200 trials were completed a multiple linear regression analysis was performed on the data, see Figure 35. A simplified general regression model can be 
seen in the equation below and the individual simplified regression equations for the eight designs can be found in Table 7.

$$
\Delta \mathrm{V}=\text { 9.96116-0.0011353Np-0.318917Random_F-0.545645High_Cr-1.61172Pruned }
$$

\section{Table 7: Multiple Regression Designs and Simplified Regression Equations}

\begin{tabular}{|c|c|c|c|c|}
\hline Design \# & Random_F & High_Cr & Pruned & Regression Equation \\
\hline 1 & 0 & 0 & 0 & $\Delta \mathrm{V}=9.961160-0.0011353 \mathrm{~Np}$ \\
\hline 2 & 0 & 0 & 1 & $\Delta \mathrm{V}=8.349440-0.0011353 \mathrm{~Np}$ \\
\hline 3 & 0 & 1 & 0 & $\Delta \mathrm{V}=9.415515-0.0011353 \mathrm{~Np}$ \\
\hline 4 & 0 & 1 & 1 & $\Delta \mathrm{V}=7.803795-0.0011353 \mathrm{~Np}$ \\
\hline 5 & 1 & 0 & 0 & $\Delta \mathrm{V}=9.642243-0.0011353 \mathrm{~Np}$ \\
\hline 6 & 1 & 0 & 1 & $\Delta \mathrm{V}=8.030523-0.0011353 \mathrm{~Np}$ \\
\hline 7 & 1 & 1 & 0 & $\Delta \mathrm{V}=9.096598-0.0011353 \mathrm{~Np}$ \\
\hline 8 & 1 & 1 & 1 & $\Delta \mathrm{V}=7.484878-0.0011353 \mathrm{~Np}$ \\
\hline
\end{tabular}

While it is important to note to that the simplified equations above ignore any of the interactions between predictor variables, they provide a straightforward way to view how they affect the $\Delta \mathrm{V}$ response. Based on these results it can be seen that individual effects of the indicators each reduce the mean $\Delta \mathrm{V}$ when all other predictor variables are ignored. Figure 35 clearly shows that utilizing a high crossover probability and pruning the search space will result in the most desirable responses. It was originally hypothesized that randomizing the mutation scaling factor would decrease the mean $\Delta \mathrm{V}$, however, the full regression analysis, which can be found in Appendix C, determined that its effects were insignificant at a 5\% significance level. This again can be seen in Figure 35 by the similarities in the regression slopes of the designs that differ only by that indicator variable. It is necessary to remember that the tests were run only through 100 iterations. One of the key advantages of randomizing the mutation scaling factor is that it 
helps avoid stagnation. Unfortunately to see this, the trials would need to be run for more than 100 iterations. Therefore, it is the recommendation of this paper that while the evidence is not shown here, the mutation scaling factor should be randomized to improve the optimization process. Conclusively, however, maintaining a higher crossover probability and using a pruned search space are highly advantageous to the optimization process.

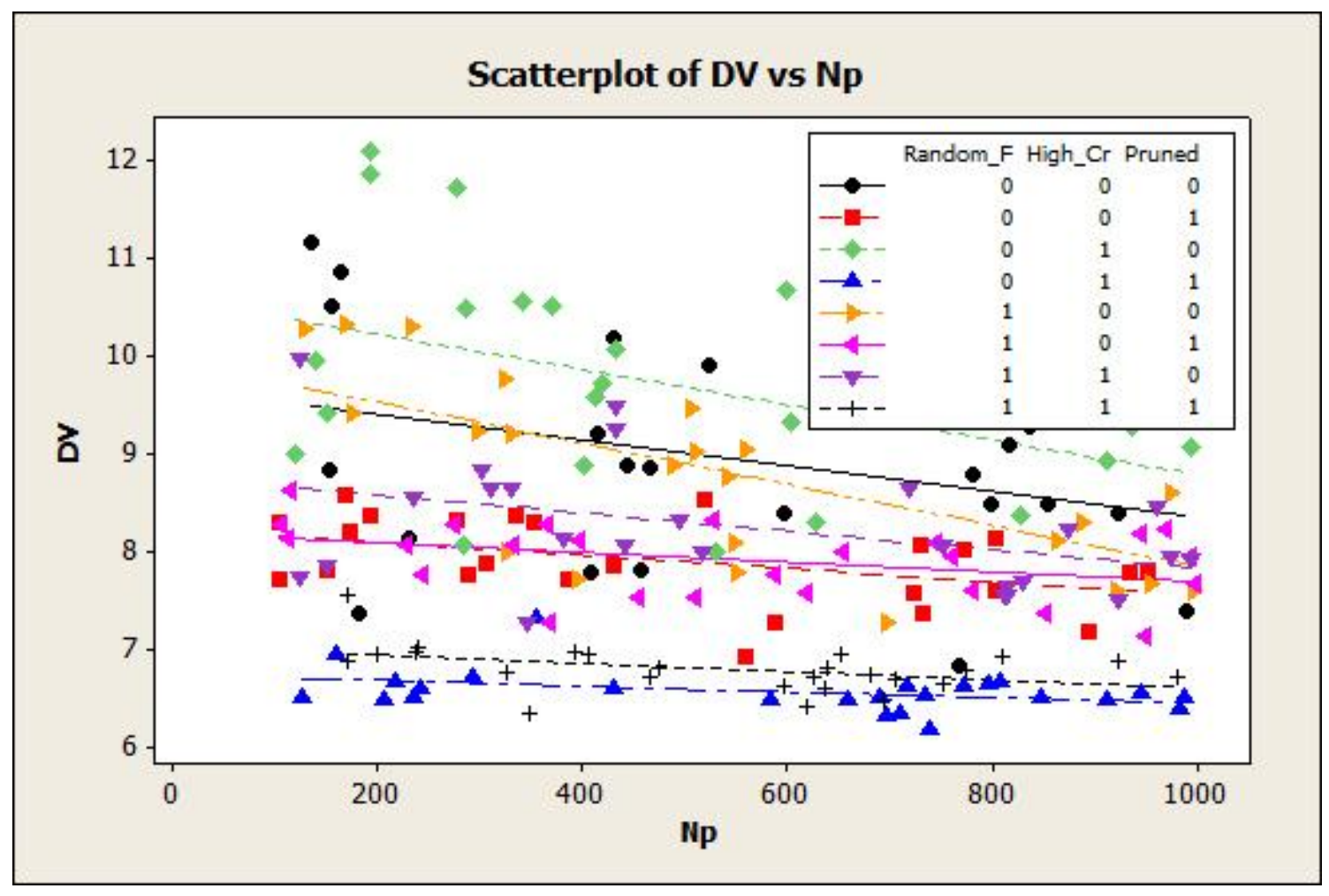

Figure 35: Multiple regression data set and regression slopes 


\section{COMET'S GRAPHICAL USER INTERFACE}

COMET's graphical user interface design was created to give users an easy and straightforward way to initialize interplanetary trajectory design problems and step through the processes of pruning the search space, optimizing to the global minimum, and visualizing trajectories. The main window consists of three panels: a title panel, a status bar panel, and the main panel. The main panel is where the majority of the user interaction occurs and to accommodate for the large number GUI elements it is broken up into four tabbed layers: Set-Up, Prune, Optimize, and Visualize.

The title panel, easily distinguishable by the COMET logo and name, allows the user to define the problem set's name as well as start a new problem set, load a preexisting set, or save the current problem. To help avoid accidental erasure from a mistaken button click, COMET always prompts the user to save the current data set before loading or opening a new scenario. If the current directory is also the root directory for the problem when the user selects to load a previous problem set, then the program automatically changes the directory to the default "Saved Data" folder where the problem set is most likely to be found and reverts back to the root directory after loading the saved information.

The status bar panel on the right-side of the window consists of two main subsets of GUI elements. The set found near the top of the window shows the user's progress as a whole in completing the following aspects of the optimization process: initializing or setting up the problem set (Set-Up), pruning the search space (Prune), optimizing through Differential Evolution (Optimize), and visualizing the optimal trajectory (Visualize). As the process is being completed, the area next to the process label will change from being 
blank to a yellow hour glass, and upon completion of the process the hour glass is replaced by a green check mark. The elements in the bottom half of the panel display the current progress within current process. As the process is being completed the progress bar and title above it show the percentage of the process completed. The elapsed time above that shows how long the process has been running. The remaining time shown at the bottom of the page is based off the elapsed time compared to the current percentage completed. During the visualization process, the time it takes to continually update the progress bar and time displays, exceeds the time it takes to make all the necessary calculations and plots. Therefore, for efficiency these elements are not updated until after the visualization process has been completed.

The first of the four tabbed layers in the main panel, Set-Up, allows the user to initialize the problem set by defining the sequence, parameter bounds, constraints and weightings. To specify the number of days for grid spacing for the pruning process, the user can select "Coarse" for 21 days, "Moderate" for 14 days, "Fine" for 7 days, or "Very Fine" for a single day. Users should be aware of the computational limits of their machine, as small grid spacing values accompanied with large search spaces can cause memory issues for MATLAB. As planets or DSM's are added or removed to the sequence, the panel automatically updates the information and toggles the visibility of the elements. If the user attempts to add a DSM as the final maneuver in the sequence or removes a planet causing the DSM to become second in the sequence, COMET notifies the user that it cannot use the current sequence and reverts to the prior sequence. Additionally, in this panel the user can specify the orbital insertion parameters. By entering a 0 in for the periapse radius, this signals the program to perform a rendezvous 
maneuver at the final planet instead of an orbit insertion. See Figure 36 for an example of how the program looks during the set-up phase of the EVVEJS problem set.

The Prune tabbed layer is the simplest of the four layers. This tab allows the user to initialize the search space pruning process and visualize both the valid search space regions, as well as the new bounds for each of the parameters. Figure 37 shows how this tab looks after the pruning process has been completed for the EVVEJS problem set.

The third tab, Optimize, gives the user the ability to specify values for the Differential Evolution optimization process, such as, the population size, the mutation scaling factor, the cross over ratio, and the preferred DE strategy. Additionally, the user can specify the stopping criteria and the frequency at which the updated information is returned. During the optimization process, this updated information displayed in the top left corner of the panel. This includes the parameter values and associated $\Delta \mathrm{V}$ 's for the best trajectory solution found so far. At the same refresh rate, the graph on the right side

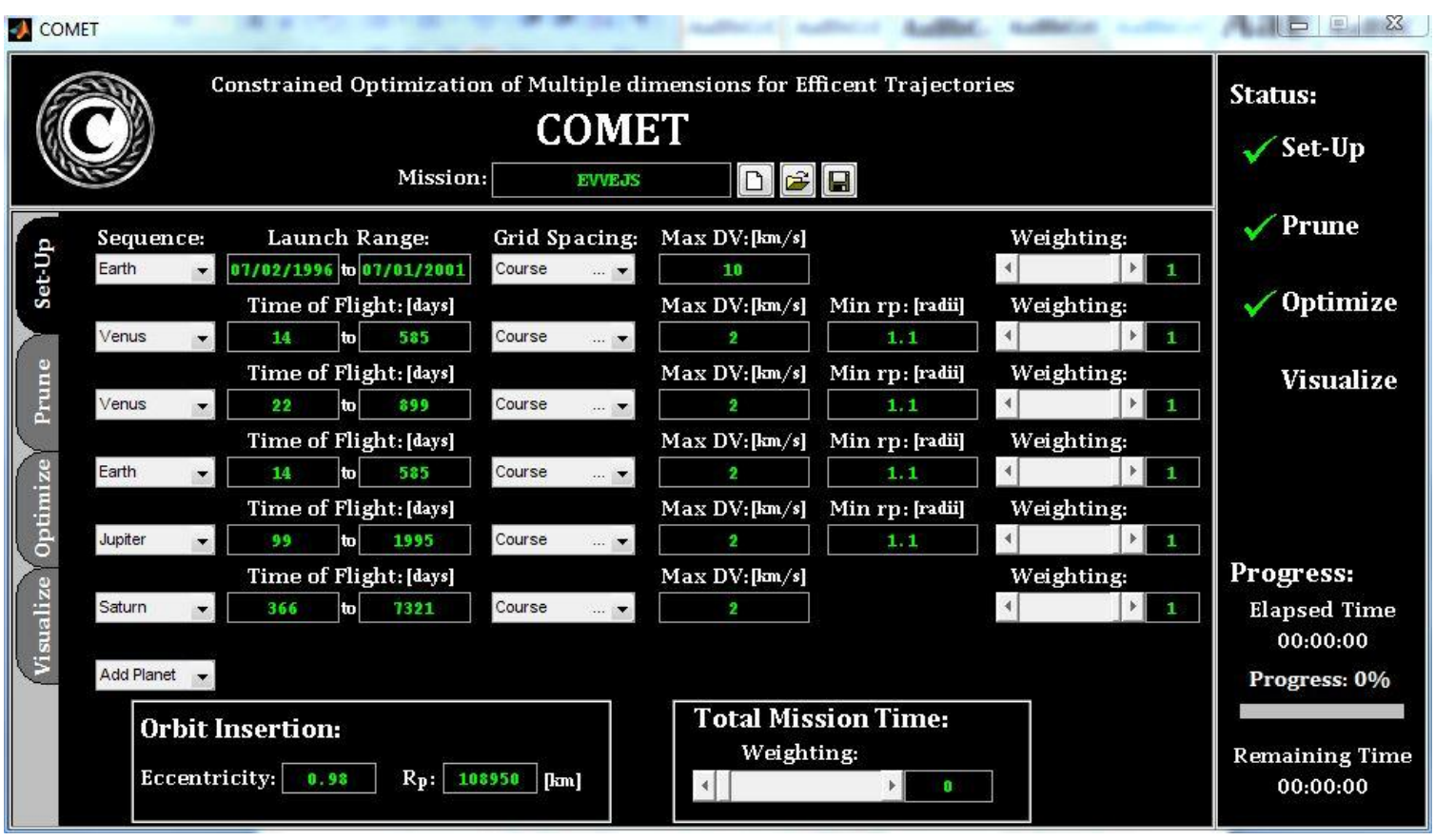

Figure 36: COMET during Set-Up for EVVEJS problem set 


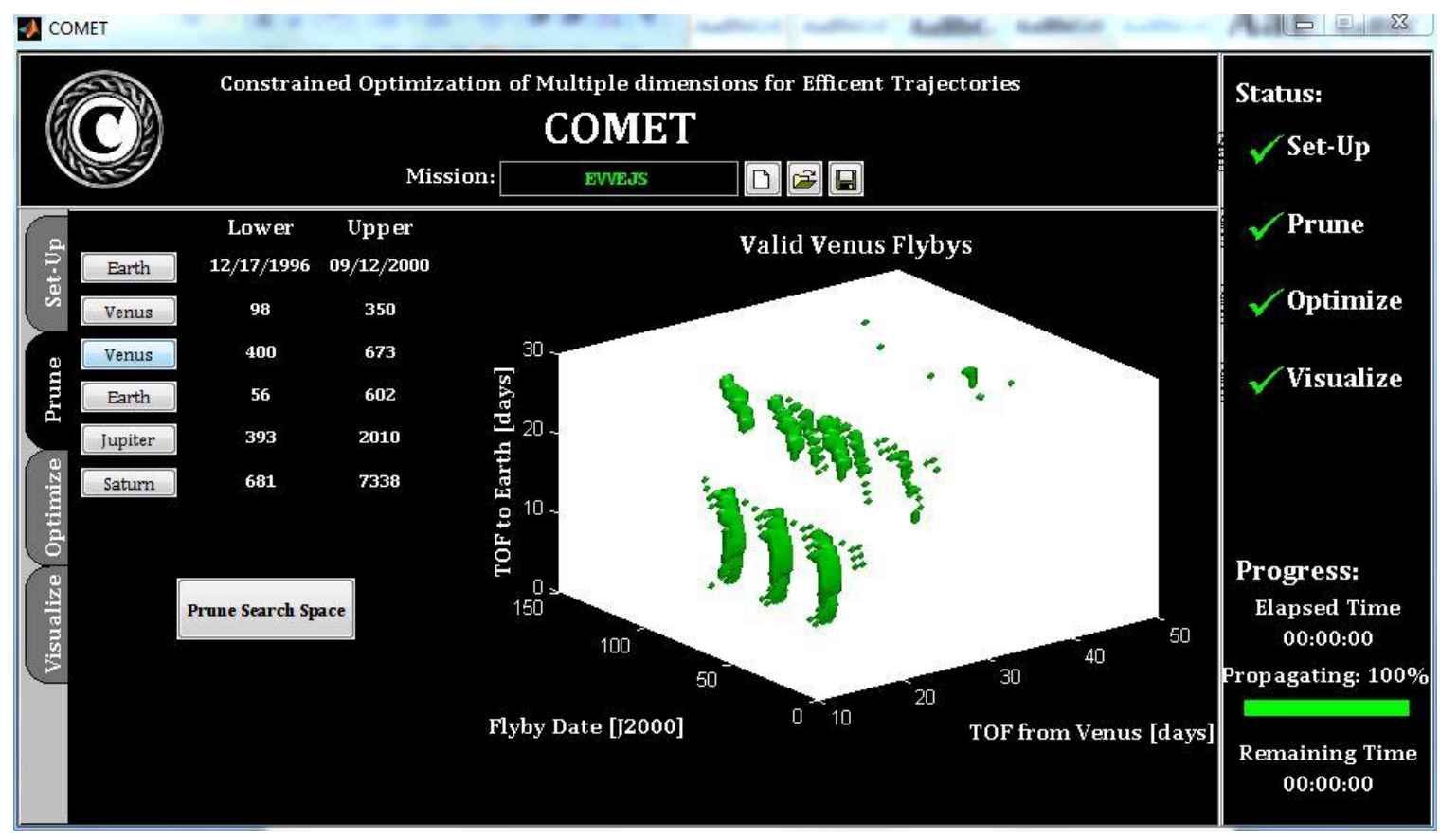

Figure 37: COMET with Prune tab selected for EVVEJS problem set

of the panel updates to show the residual plot of the total $\Delta \mathrm{V}$ associated with the best found solution for that iteration. As the solution converges, the residual plot will flatten out. After the solution converges and the program restarts the population, the ultimate best found solution is kept separated from the current population. Therefore, each time the population restarts it can be seen as a spike in the graph. Figure 38 shows this tabbed layer after the optimization process for the EVVEJS problem set.

The Visualize tab is the final layer. Initially, COMET plots the orbits of the planets and the trajectory of the spacecraft using the parameters from the best known solution. Similar to the Optimization tab, the parameters and associated $\Delta V$ 's can be found in the upper left-hand corner of the panel. However, unlike the previous tab, here the parameters can be modified by the user and to view the effects on the trajectory and cost. The user has the additional option to select a specific date and time within the current trajectory's mission to view the position of the spacecraft and planets at that time. 


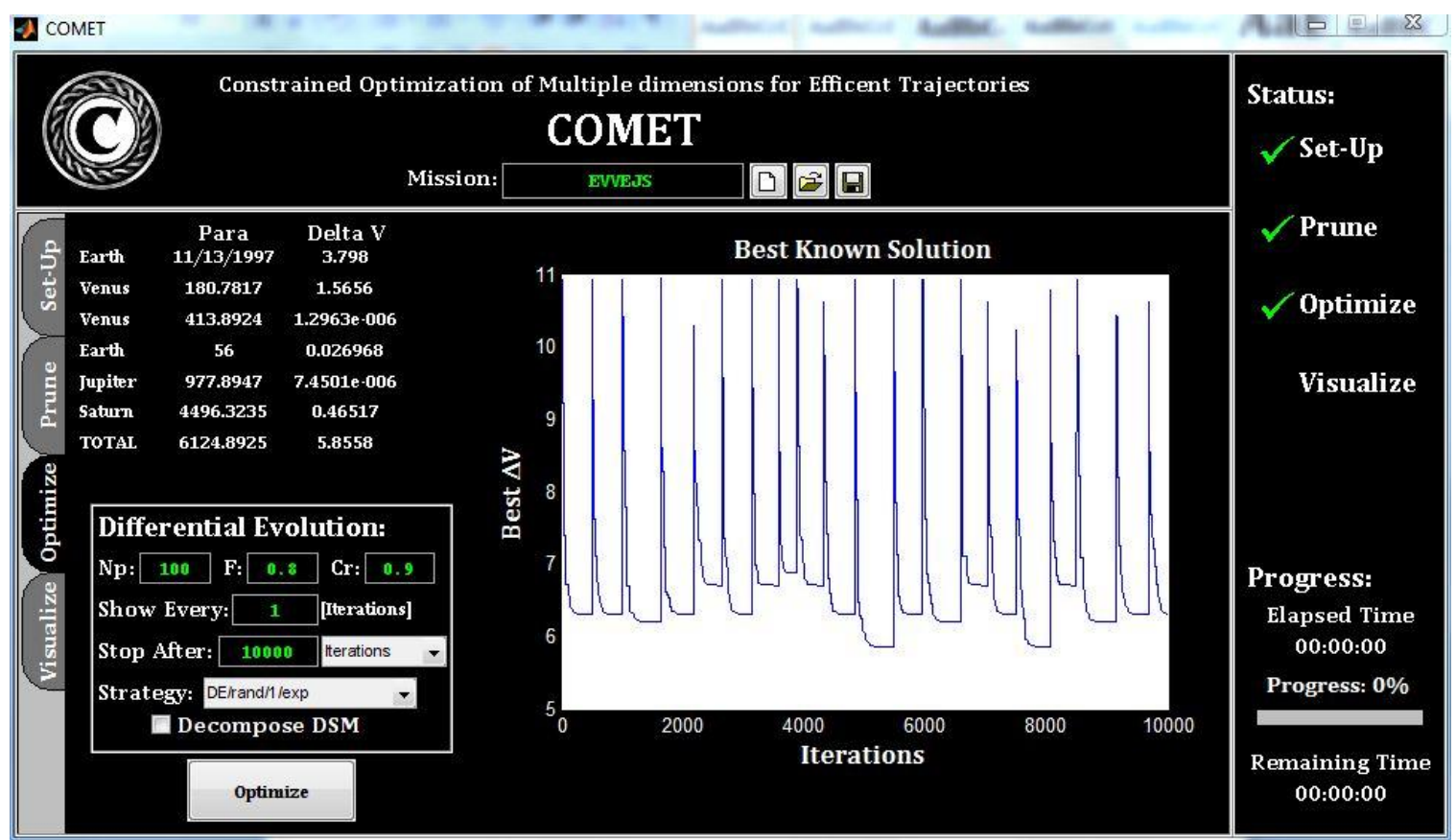

Figure 38: COMET with Optimization tab selected for EVVEJS problem set

By pressing the "Launch" button, the user can watch the animation of the spacecraft as it completes the mission. While the spacecraft is within the sphere of influence of a planet during a flyby, the view shifts to a planet-centric frame, and then back to the helio-centric view once the spacecraft leaves that sphere of influence. By default, only the orbits of the planets closer to the sun than the farthest planet in the sequence are visible. However, the user has the option to toggle the orbits of any planets to make viewing the trajectory cleaner or more informative. Finally, the user can select from one of the three small push buttons in the lower left-hand corner, enabling them to pan, orbit, or zoom in on the current view. By selecting the "Reset" button, all views and dates are reset to the final date using the best known solution. This visualization can be seen in Figure 39, which demonstrates how COMET would initially display the orbits for the best known solution to the EVVEJS problem set. 


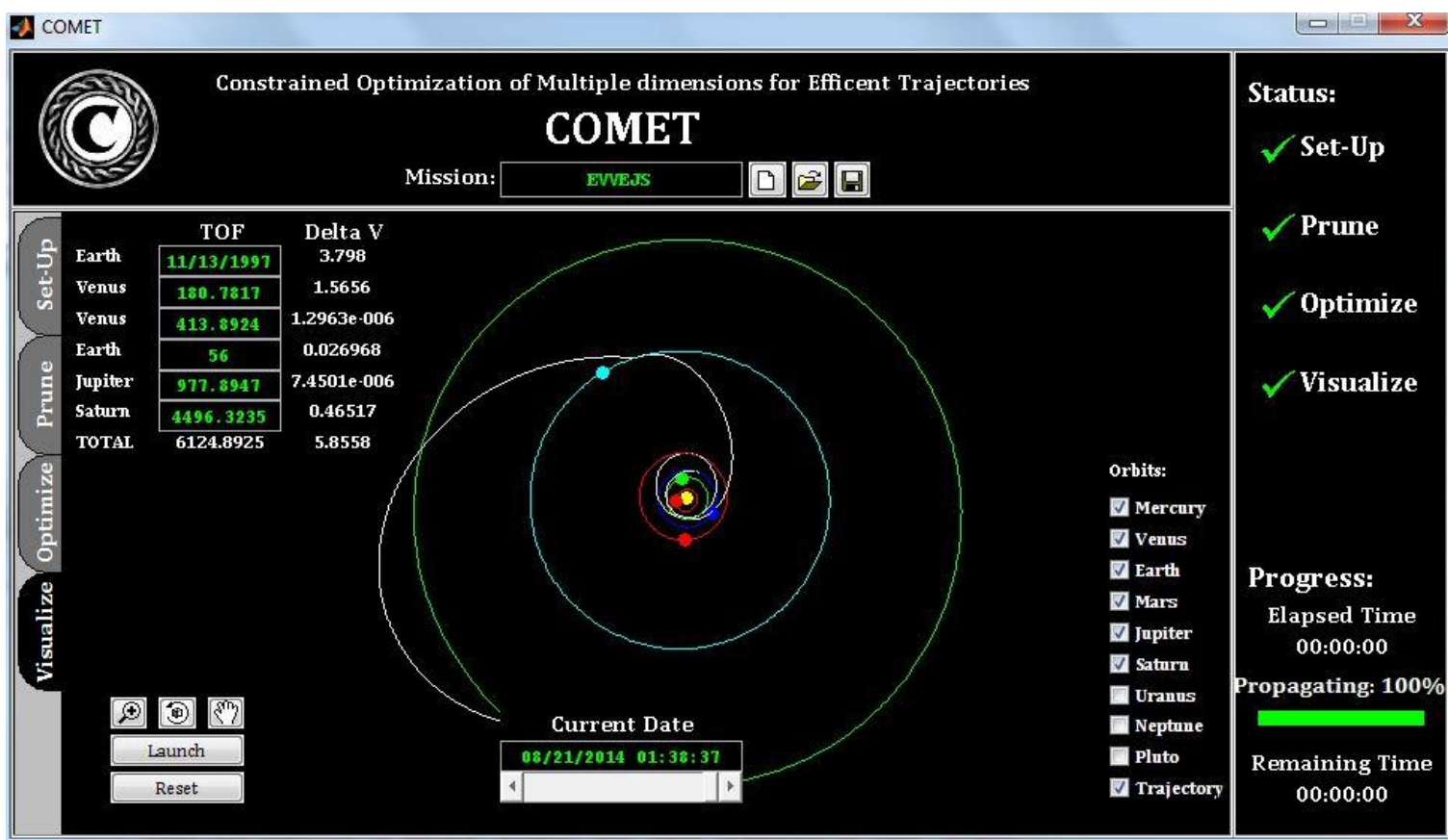

Figure 39: COMET with Visualize tab selected for EVVEJS problem set 


\section{CONCLUSION AND FUTURE WORK}

An evaluation of the program COMET, Constrained Optimization of Multipledimensions for Efficient Trajectories, was performed and compared against the results previously found by the European Space Agency's MGA program from its Advanced Concepts Team. COMET utilized an efficient cascading grid sampling technique to prune multiple dimension interplanetary trajectory problem search spaces to improve the quality of the initial optimization population and reduce the upper and lower bounds placed on each dimension. Additionally, recognizing the nature of trajectory problems to be in general not decomposable, COMET made use of a higher cross-over probability factor, $\mathrm{CR}$, and randomized mutation scaling factor, $\mathrm{F}$, in its Differential Evolution global optimization algorithm, which increased the convergence rate for each trial. After comparing COMET to MGA and additionally running a multiple linear regression analysis it was concluded that these improvements established COMET to have increased robustness while maintaining and in some cases improving the level of accuracy. Table 8 shows the condensed results for all five test cases.

Table 8: Test case results in $\mathrm{km} / \mathrm{s}$

\begin{tabular}{|l|c|c|c|}
\hline Test Case & MGA's Best & $\begin{array}{c}\text { MGA's Best ran } \\
\text { w/ COMET }\end{array}$ & COMET's Best \\
\hline EJS & 9.35179 & 9.40844 & 9.35405 \\
\hline EMJS & 9.18597 & 10.05749 & 9.94655 \\
\hline EVEJS & 7.54837 & 9.77233 & 6.18321 \\
\hline EVVEJS & 6.14199 & 6.68801 & 5.85582 \\
\hline EVDVEJS & 4.58906 & 5.03234 & 4.79272 \\
\hline EVDVEJS decomp & 4.58906 & 5.03234 & 4.75262 \\
\hline
\end{tabular}

In all five test cases, COMET and MGA showed differing values between the calculated costs associated with individual trajectories. Further investigation showed that the root of the discrepancies revolved around the evaluation of flybys. Here, COMET 
calculated the change in velocities based off a minimum altitude flyby, and MGA calculated the necessary altitude for the given flyby and added a penalty factor for altitudes below the minimum allowable value. To mitigate these differences and directly compare the results, MGA's solutions were processed by COMET's cost function. In all five cases, COMET was able to locate solutions that produced lower $\Delta \mathrm{V}$ outcomes than the MGA solutions. Furthermore, the application of search space pruning consistently led COMET to have a higher convergence rate, and in the last two cases, COMET located highly isolated solutions that had not been discovered by the MGA program, making it a more ideal program for interplanetary trajectory design.

To further aid mission designers, later versions of COMET will include the option for multiple revolution trajectory branches. This stands to expand the valid search space regions without increasing the dimensionality of search space. Additionally, COMET will implement various modern control techniques to approximate the necessary propellant for trajectory correction maneuvers in the presence of an n-body gravitational field. These will include a fully modulated thruster scheme for preliminary analysis, a Schmitt Trigger scheme to allow for error bounds to be applied to the nominal trajectory and reduce fuel consumption, and finally a novel forward looking relay scheme to further reduce propellant expenditures. It has also been suggested to replace the individual weights with maneuver Isp's, and evaluate each trajectory based on the spacecraft's final mass fraction. Future models may also include the possibility of changing the central body and adding custom objects, such as moons and asteroids. Investigations into methods to prune DSM parameters may lead to further refinement in later versions. Finally, consideration into allowing multiple sequential deep space maneuvers between planets and utilizing 
electric propulsion options for prolonged thrust arcs may be included in later versions. These modifications will open the possibilities for affordable options in exploring planets in our solar system. 


\section{REFERENCES}

1. "ACT - Informatics." ESA Communications Portal. Web. 18 Nov. 2011. <http://www.esa.int/gsp/ACT/inf/op/globopt/mgadsm.htm>.

2. Battin, Richard H. An Introduction to the Mathematics and Methods of Astrodynamics. Reston, VA: American Institute of Aeronautics and Astronautics, 1987. Print.

3. Curtis, Howard D. Orbital Mechanics for Engineering Students. Amsterdam: ButterworthHeinemann, 2005. Print.

4. Dowling, Richard L.; Kosmann, William J.; Minovitch, Michael A.; and Ridenoure, Rex W., "The origin of gravity-propelled interplanetary space travel" (IAA paper no. 90-630), presented at the 41st Congress of the International Astronautical Federation, which was held 6-12 October 1990 in Dresden, G.D.R.

5. Gooding, R.H. "A procedure for the solution of Lambert's orbital boundary-value problem." Celestial Mechanics and Dynamical Astronomy, 48:145-165,1990.

6. Izzo, D. ESA Advanced Concepts team. Code used available in MGA.M, on http://www.esa.int/gsp/ACT/inf/op/globopt.htm. Last retreived Dec, 2010.

7. Lancaster, E.R. and Blanchard, R.C. "A unified form of Lambert's theorem." NASA technical note TN D-5368,1969.

8. Myatt, D.R., Becerra, V.M., Nasuto, S.J., Bishop, J.M.: Advanced global optimisation for mission analysis and design. Final Report. Ariadna id: 03/4101. Contract Number: 18138/04/NL/MV, 2004. Available: http://www.esa.int/gsp/ACT/doc/ACT-RPT-03-4101ARIADNA-Global OptimisationReading. pdf.

9. Ochoa, S. I., and Prussing, J. E., " Multiple Revolution Solutions to Lambert's Problem ", Advances in the Astronautical Sciences, Vol. 79, Part II, Eds. B. Kaufman et al., Univelt Inc., San Diego, CA. [ Presented as Paper No. 92-194, AAS/AIAA Spaceflight Mechanics Meeting, Colorado Springs CO, February 1992.]

10. Price, Kenneth V., Storn, Rainer M., and Lampinen, Jouni A.. Differential Evolution: a Practical Approach to Global Optimization. Berlin: Springer, 2005. Print.

11. Prussing, John E., and Conway, Bruce A. Orbital Mechanics. New York: Oxford UP, 1993. Print.

12. Schaub, Hanspeter, and Junkins. Analytical Mechanics of Space Systems. Reston, VA: American Institute of Aeronautics and Astronautics, 2003. Print.

13. Turner, Martin J. L. Rocket and Spacecraft Propulsion: Principles, Practice and New Developments. Berlin: Springer, 2009. Print.

14. Vallado, David A., and McClain, Wayne D. Fundamentals of Astrodynamics and Applications. Dordrecht: Kluwer Academic, 2001. Print.

15. Wertz, James Richard., and Larson, Wiley J. Space Mission Analysis and Design. El Segundo, Calif.: Microcosm, 1999. Print. 


\section{APPENDIX A: INITIAL PROBLEM STRUCTURES}

\section{Initial problem structure for EJS}

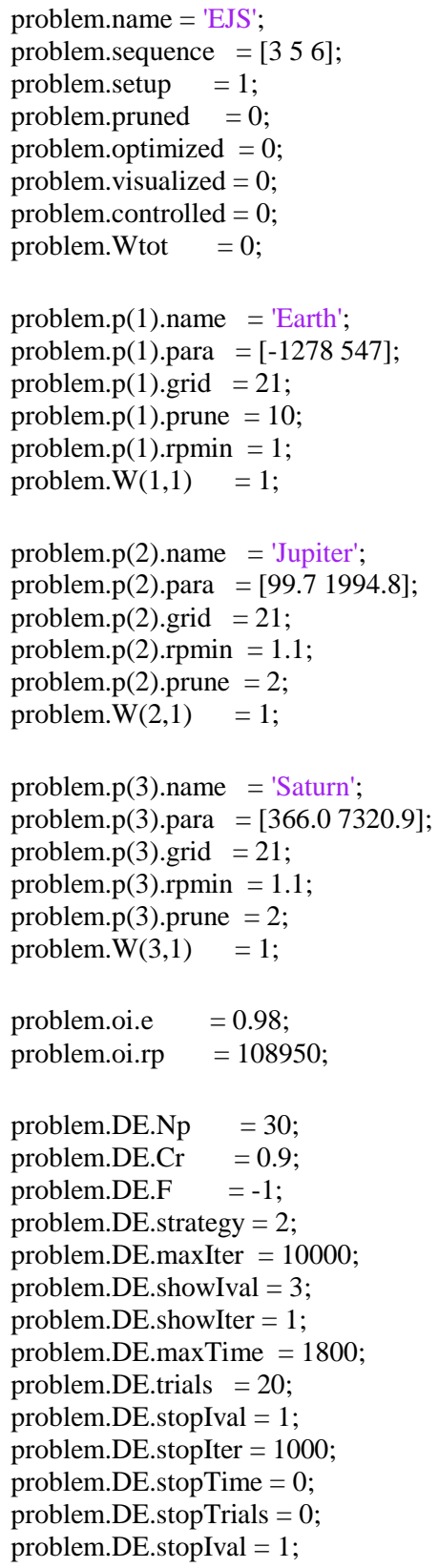

\section{Initial problem structure for EMJS}

problem. name $=$ 'EMJS';

problem.sequence $=\left[\begin{array}{lll}3 & 4 & 5\end{array}\right]$; 


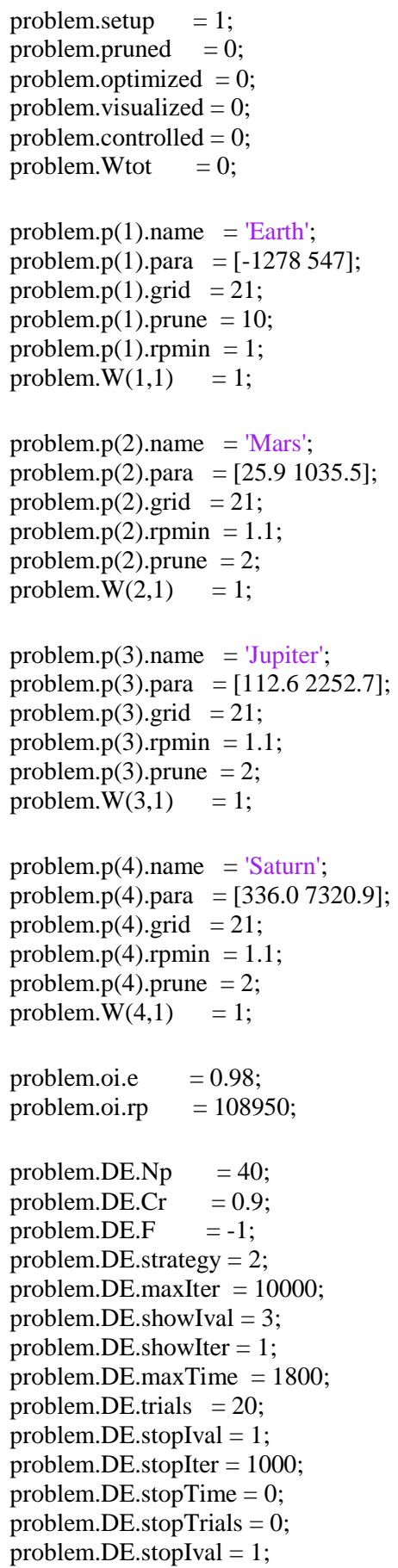

\section{Initial problem structure for EVEJS}

problem.name = 'EVEJS';

problem.sequence $=\left[\begin{array}{lllll}3 & 2 & 3 & 5 & 6\end{array}\right]$;

problem.setup $=1$;

problem.pruned $=0$;

problem.optimized $=0$

problem. visualized $=0$;

problem. controlled $=0$;

problem. Wtot $=0$;

problem.p(1).name = 'Earth'; 


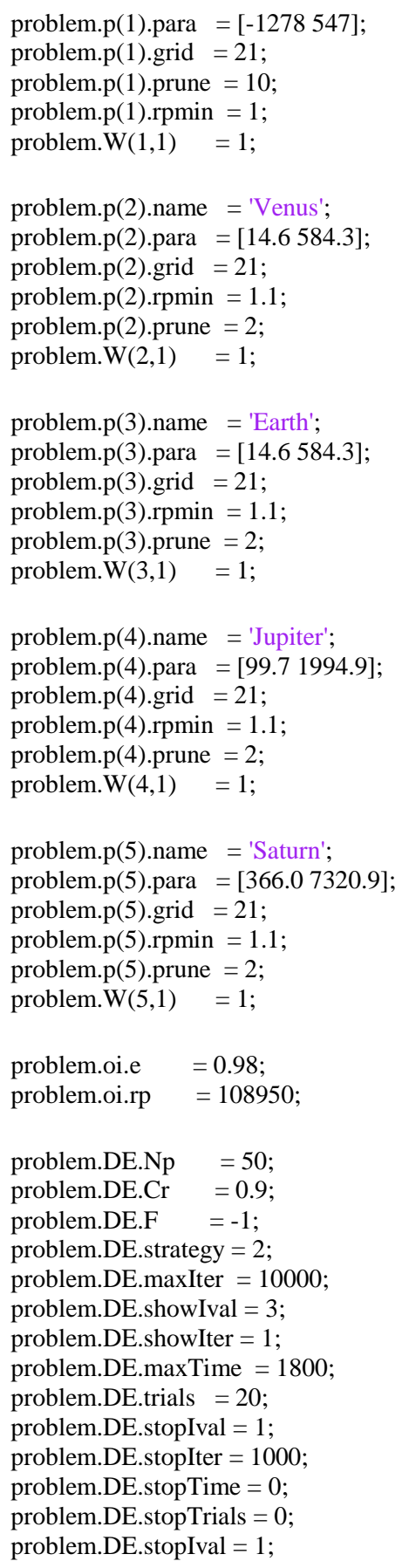

\section{Initial problem structure for EVVEJS}

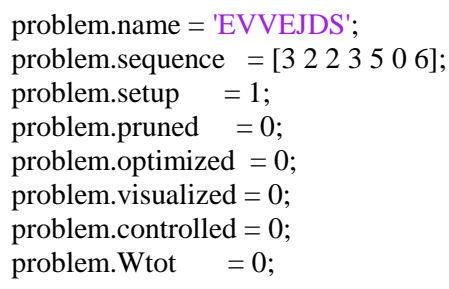




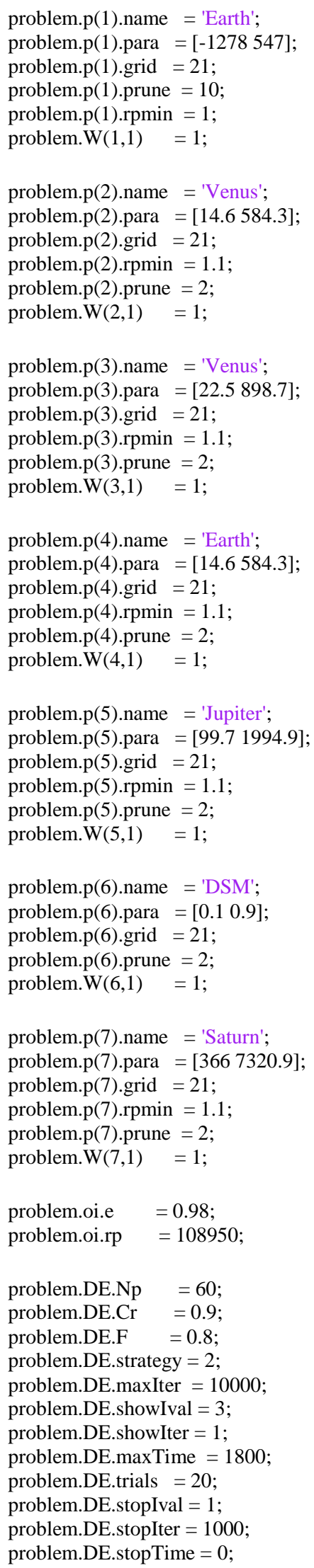


problem.DE. stopTrials $=0$;

problem.DE.stopIval $=1$; 


\section{Appendix B: COMET's Lambert's Problem Function}

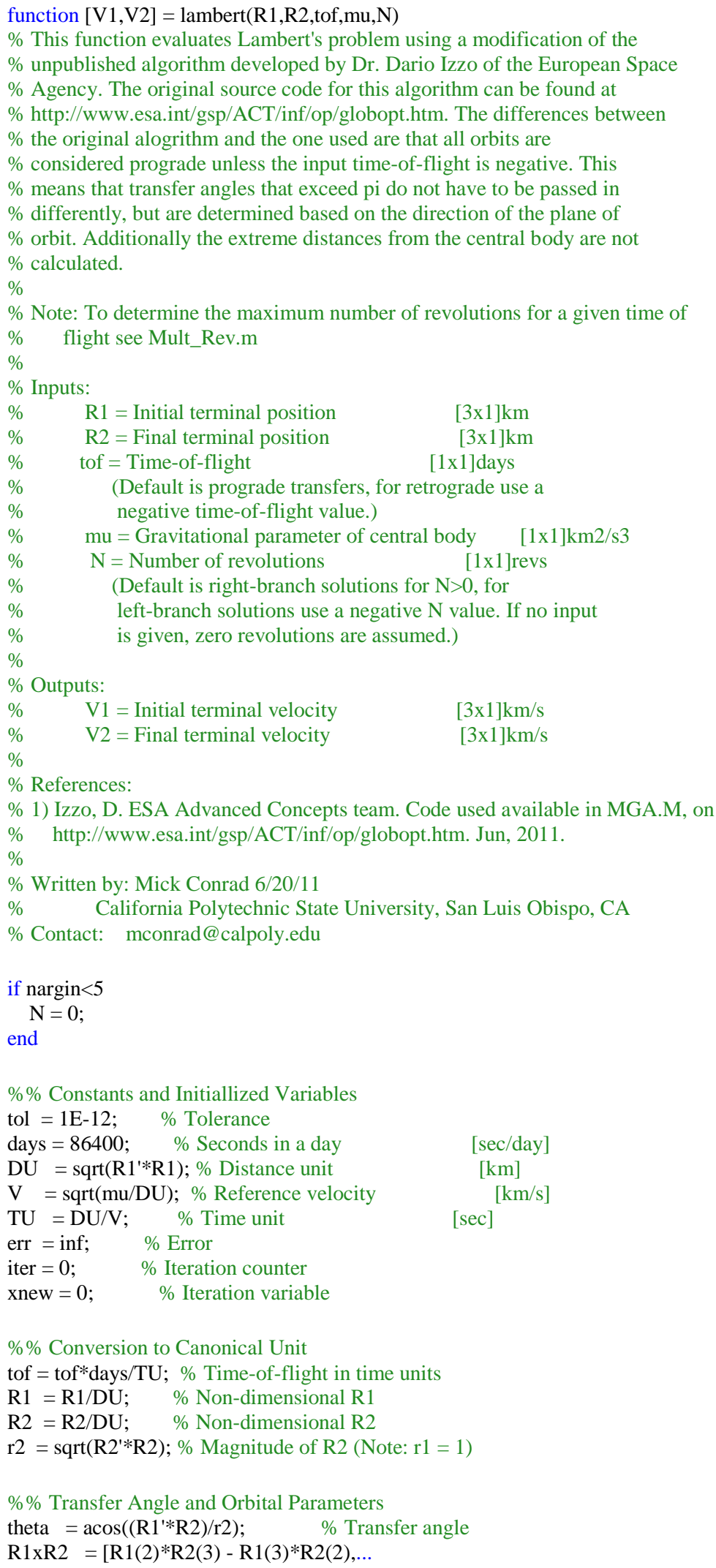




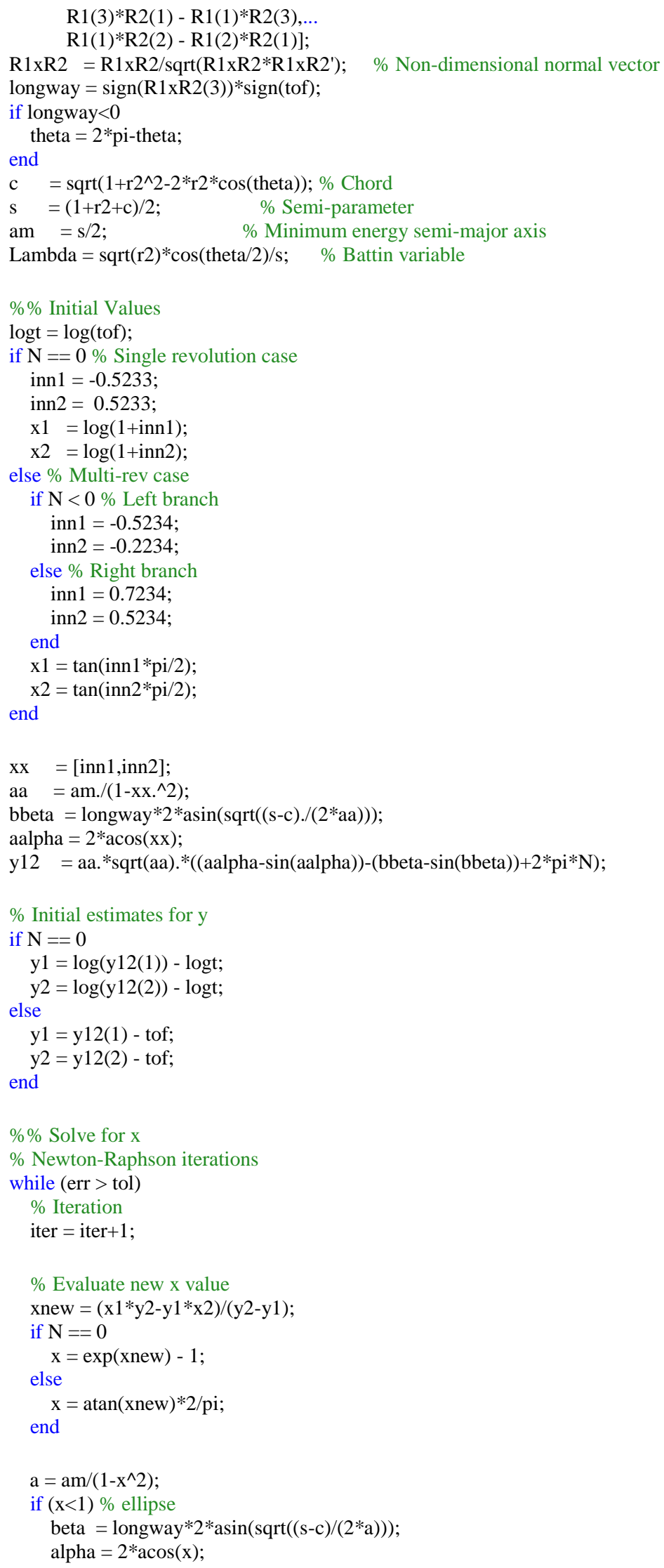




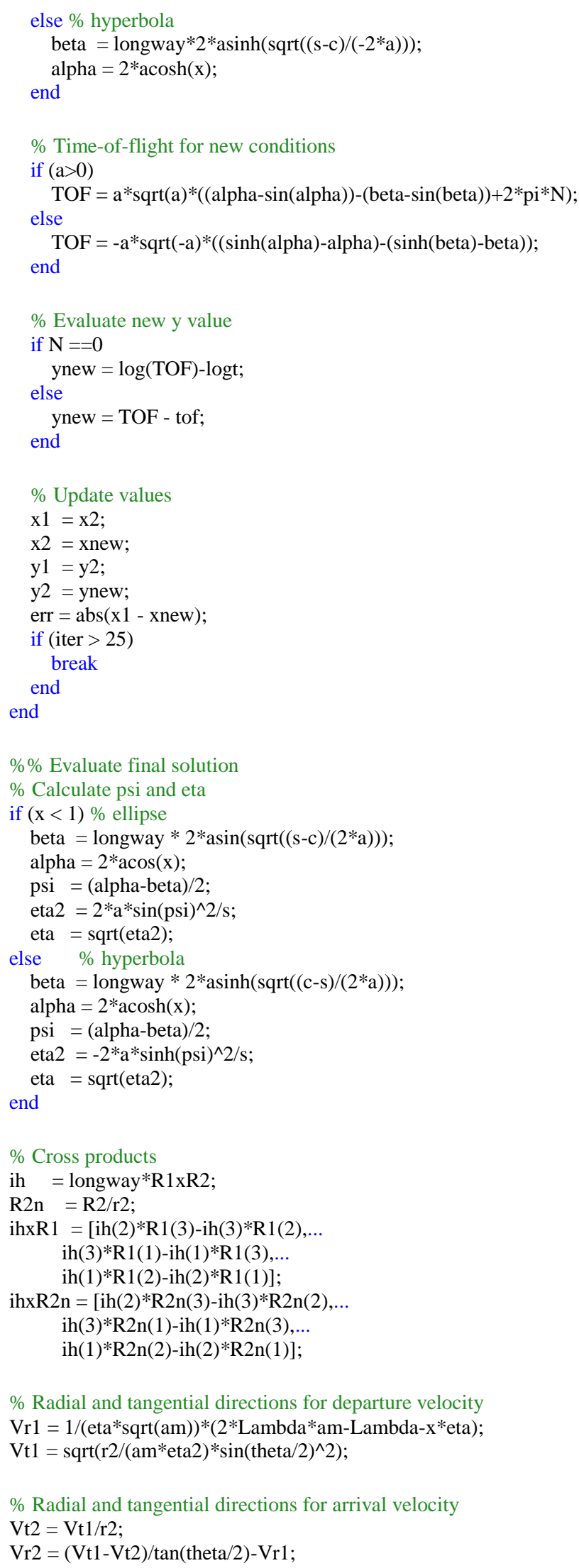


$\%$ Terminal velocities

$\mathrm{V} 1=\left(\mathrm{Vr} 1 * \mathrm{R} 1+\mathrm{Vt} 1{ }^{*} \mathrm{ihxR} 1{ }^{\prime}\right) * \mathrm{~V}$;

$\mathrm{V} 2=\left(\mathrm{Vr} 2 * \mathrm{R} 2 \mathrm{n}+\mathrm{Vt} 2 * \mathrm{~h} \times \mathrm{R} 2 \mathrm{n}^{\prime}\right) * \mathrm{~V}$;

end 


\title{
APPENDIX C: MULTIPLE LINEAR REGRESSION ANALYSIS
}

\author{
1. Full Model
}

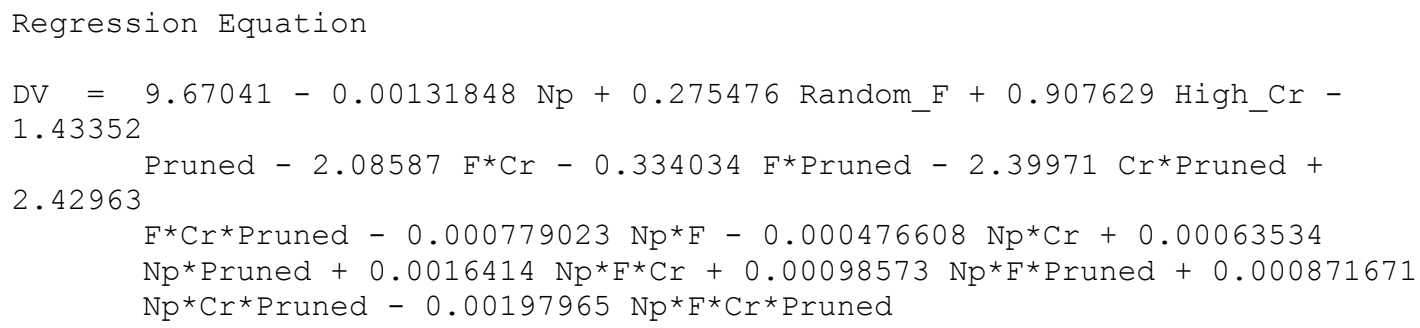

\begin{tabular}{|c|c|c|c|c|c|}
\hline Term & Coef & SE Coef & $\mathrm{T}$ & $\mathrm{P}$ & VIF \\
\hline Constant & 9.67041 & 0.293948 & 32.8984 & 0.000 & \\
\hline $\mathrm{Np}$ & -0.00132 & 0.000476 & -2.7670 & 0.006 & 7.9574 \\
\hline Random_F & 0.27548 & 0.420204 & 0.6556 & 0.513 & 20.6124 \\
\hline High_C $\bar{r}$ & 0.90763 & 0.403936 & 2.2470 & 0.026 & 19.0473 \\
\hline Prunèd & -1.43352 & 0.396814 & -3.6126 & 0.000 & 18.3815 \\
\hline $\mathrm{F}^{\star} \mathrm{Cr}$ & -2.08587 & 0.580682 & -3.5921 & 0.000 & 29.5221 \\
\hline$F^{*}$ Pruned & -0.33403 & 0.571717 & -0.5843 & 0.560 & 28.6176 \\
\hline Cr*Pruned & -2.39971 & 0.574020 & -4.1805 & 0.000 & 28.8486 \\
\hline $\mathrm{F} * \mathrm{Cr} *$ Pruned & 2.42963 & 0.832884 & 2.9171 & 0.004 & 35.4287 \\
\hline $\mathrm{Np} * \mathrm{~F}$ & -0.00078 & 0.000680 & -1.1449 & 0.254 & 24.5266 \\
\hline $\mathrm{Np} * \mathrm{Cr}$ & -0.00048 & 0.000689 & -0.6922 & 0.490 & 24.5346 \\
\hline Np *Pruned & 0.00064 & 0.000671 & 0.9464 & 0.345 & 23.4936 \\
\hline $\mathrm{Np} * \mathrm{~F} * \mathrm{Cr}$ & 0.00164 & 0.000959 & 1.7112 & 0.089 & 31.8395 \\
\hline$N p * F *$ Pruned & 0.00099 & 0.000942 & 1.0464 & 0.297 & 30.5708 \\
\hline $\mathrm{Np} * \mathrm{Cr} *$ Pruned & 0.00087 & 0.000959 & 0.9090 & 0.365 & 33.0042 \\
\hline $\mathrm{Np} * \mathrm{~F} * \mathrm{Cr} *$ Pruned & -0.00198 & 0.001374 & -1.4412 & 0.151 & 34.0904 \\
\hline \multicolumn{6}{|l|}{ Summary of Model } \\
\hline$S=0.654456$ & \multirow{2}{*}{\multicolumn{2}{|c|}{$\begin{array}{l}\mathrm{R}-\mathrm{Sq}=73.27 \% \\
\mathrm{R}-\mathrm{Sq}(\text { pred })=68.29\end{array}$}} & \multirow{2}{*}{\multicolumn{3}{|c|}{$R-S q(\operatorname{adj})=71.09 \%$}} \\
\hline PRESS $=93.4856$ & & & & & \\
\hline
\end{tabular}

Analysis of Variance

$\begin{array}{lrrrrrr}\text { Source } & \text { DF } & \text { Seq SS } & \text { Adj SS } & \text { Adj MS } & \text { F } & \text { P } \\ \text { Regression } & 15 & 215.989 & 215.989 & 14.3993 & 33.6185 & 0.000000 \\ \quad \text { Np } & 1 & 21.565 & 3.279 & 3.2794 & 7.6564 & 0.006235 \\ \text { Random_F } & 1 & 5.049 & 0.184 & 0.1841 & 0.4298 & 0.512915 \\ \text { High_Cr } & 1 & 14.872 & 2.162 & 2.1625 & 5.0488 & 0.025831 \\ \quad \text { Pruned } & 1 & 129.872 & 5.590 & 5.5898 & 13.0507 & 0.000391 \\ \text { F*Cr } & 1 & 3.991 & 5.527 & 5.5266 & 12.9032 & 0.000421 \\ \text { F*Pruned } & 1 & 9.968 & 0.146 & 0.1462 & 0.3414 & 0.559759 \\ \text { Cr*Pruned } & 1 & 19.556 & 7.486 & 7.4855 & 17.4768 & 0.000045 \\ F^{*} \text { Cr*Pruned } & 1 & 5.427 & 3.645 & 3.6448 & 8.5096 & 0.003973 \\ \text { Np*F } & 1 & 0.000 & 0.561 & 0.5615 & 1.3109 & 0.253725 \\ \text { Np*Cr } & 1 & 0.216 & 0.205 & 0.2052 & 0.4791 & 0.489705 \\ \text { Np*Pruned } & 1 & 4.153 & 0.384 & 0.3836 & 0.8957 & 0.345191 \\ \text { Np*F*Cr } & 1 & 0.420 & 1.254 & 1.2542 & 2.9282 & 0.088730 \\ \quad \text { Np*F*Pruned } & 1 & 0.003 & 0.469 & 0.4690 & 1.0949 & 0.296763 \\ \text { Np*Cr*Pruned } & 1 & 0.008 & 0.354 & 0.3539 & 0.8264 & 0.364516 \\ \quad \text { Np*F*Cr*Pruned } & 1 & 0.890 & 0.890 & 0.8897 & 2.0771 & 0.151223 \\ \text { Error } & 184 & 78.810 & 78.810 & 0.4283 & & \end{array}$




$\begin{array}{lrrrrrr}\text { Lack-of-Fit } & 182 & 76.144 & 76.144 & 0.4184 & 0.3139 & 0.956350 \\ \text { Pure Error } & 2 & 2.666 & 2.666 & 1.3330 & & \\ \text { Total } & 199 & 294.798 & & & & \end{array}$

$\begin{array}{rrrrrrr}\text { Fits and Diagnostics for Unusual Observations } \\ \text { Obs } & \text { DV } & \text { Fit } & \text { SE Fit } & \text { Residual } & \text { St Resid } \\ 10 & 12.0803 & 10.2316 & 0.197763 & 1.84868 & 2.96329 & \mathrm{R} \\ 59 & 6.8305 & 8.6578 & 0.166404 & -1.82731 & -2.88699 & \mathrm{R} \\ 64 & 11.8573 & 10.2298 & 0.197391 & 1.62751 & 2.60828 & \mathrm{R} \\ 111 & 11.7087 & 10.0790 & 0.168428 & 1.62970 & 2.57696 & \mathrm{R} \\ 115 & 10.8435 & 9.4542 & 0.226664 & 1.38936 & 2.26298 & \mathrm{R} \\ 127 & 7.8014 & 9.1325 & 0.147865 & -1.33110 & -2.08789 & \mathrm{R} \\ 139 & 7.3635 & 9.4304 & 0.219718 & -2.06697 & -3.35290 & \mathrm{R} \\ 166 & 11.1635 & 9.4924 & 0.238080 & 1.67108 & 2.74120 & \mathrm{R} \\ 174 & 7.7260 & 9.1195 & 0.152821 & -1.39350 & -2.18979 & \mathrm{R} \\ 184 & 9.9703 & 8.6511 & 0.239889 & 1.31921 & 2.16652 & \mathrm{R} \\ 188 & 8.0698 & 10.0700 & 0.166875 & -2.00022 & -3.16079 & \mathrm{R} \\ 194 & 8.9899 & 10.3626 & 0.226243 & -1.37273 & -2.23532 & \mathrm{R} \\ 199 & 8.0079 & 9.6266 & 0.132302 & -1.61878 & -2.52562 & \mathrm{R}\end{array}$

$\mathrm{R}$ denotes an observation with a large standardized residual.

\section{Reduced Model}

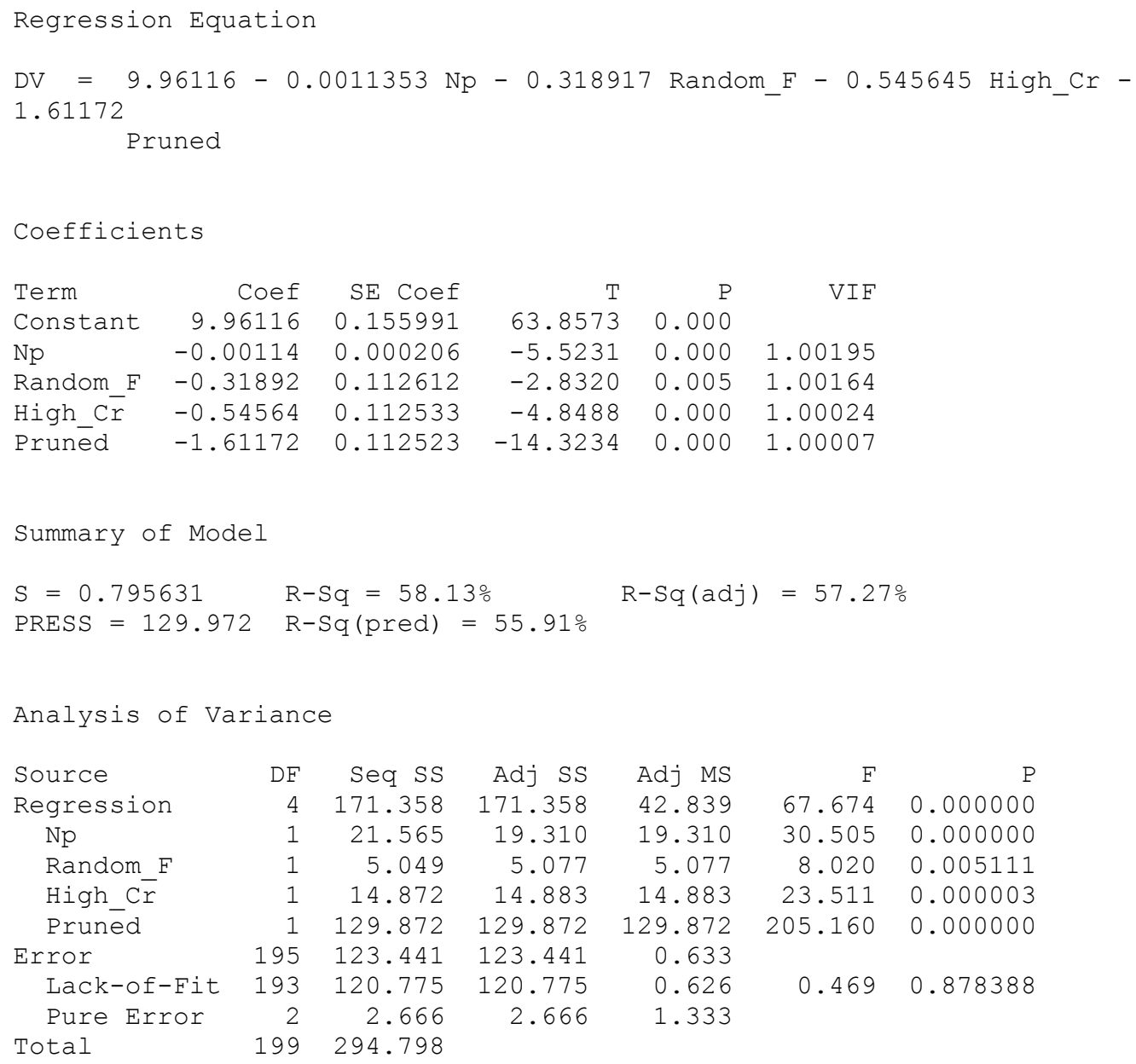




$\begin{array}{rrrrrrr}\text { Fits and Diagnostics for Unusual } & \text { Observations } \\ \text { Obs } & \text { DV } & \text { Fit } & \text { SE Fit } & \text { Residual } & \text { St Resid } & \\ 2 & 7.8136 & 9.44346 & 0.113425 & -1.62982 & -2.06960 & \mathrm{R} \\ 10 & 12.0803 & 9.19640 & 0.132570 & 2.88387 & 3.67602 & \mathrm{R} \\ 59 & 6.8305 & 9.08924 & 0.123058 & -2.25874 & -2.87351 & \mathrm{R} \\ 64 & 11.8573 & 9.19526 & 0.132462 & 2.66204 & 3.39317 & \mathrm{R} \\ 88 & 10.6786 & 8.73660 & 0.113285 & 1.94202 & 2.46598 & \mathrm{R} \\ 111 & 11.7087 & 9.09990 & 0.124220 & 2.60881 & 3.31962 & \mathrm{R} \\ 127 & 7.8014 & 9.49795 & 0.115087 & -1.69658 & -2.15504 & \mathrm{R} \\ 139 & 7.3635 & 9.75453 & 0.132849 & -2.39106 & -3.04802 & \mathrm{R}\end{array}$

$\mathrm{R}$ denotes an observation with a large standardized residual. 